Supporting Information

For

“Alkylidene Dihydropyridines as Synthetic Intermediates: Model Studies Toward the Synthesis of the Bis(piperidine) Alkaloid Xestoproxamine C”

Ashabha I. Lansakara, S. V. Santhana Mariappan, and F. Christopher Pigge*

Department of Chemistry, University of Iowa, Iowa City, Iowa 52242

\title{
Contents:
}

Crystallographic details and data 


\section{X-ray Crystallography}

Single crystals of $59 \cdot \mathrm{HCl}$ were grown from slow evaporation of a $1: 1$ mixture of $\mathrm{CHCl}_{3}$ and EtOAc. A suitable crystal was selected and mounted on a diffractometer equipped with a CCD detector. The crystal was kept at $190.15 \mathrm{~K}$ during data collection. Using Olex2, the structure was solved with the ShelXT structure solution program using Direct Methods and refined with the ShelXL refinement package using Least Squares minimisation. ${ }^{1-3}$

1. Dolomanov, O.V., Bourhis, L.J., Gildea, R.J, Howard, J.A.K. \& Puschmann, H. (2009), J. Appl. Cryst. 42, 339-341.

2. Sheldrick, G.M. (2015). Acta Cryst. A71, 3-8.

3. Sheldrick, G.M. (2015). Acta Cryst. C71, 3-8.

Table S1. Crystallographic data for $59 \cdot \mathrm{HCl}$

\begin{tabular}{|c|c|}
\hline $\mathrm{CCDC}$ & 1481779 \\
\hline Empirical formula & $\mathrm{C}_{20} \mathrm{H}_{31} \mathrm{ClN}_{2} \mathrm{O}$ \\
\hline Formula weight & 350.92 \\
\hline Temperature/K & 190.15 \\
\hline Crystal system & monoclinic \\
\hline Space group & $\mathrm{P} 2{ }_{1} / \mathrm{c}$ \\
\hline $\mathrm{a} / \AA$ & 19.0891(19) \\
\hline $\mathrm{b} / \AA$ & $10.1206(10)$ \\
\hline $\mathrm{c} / \AA$ & $9.7640(10)$ \\
\hline$\alpha /^{\circ}$ & 90 \\
\hline$\beta /{ }^{\circ}$ & $95.083(5)$ \\
\hline$\gamma /{ }^{\circ}$ & 90 \\
\hline Volume $/ \AA^{3}$ & $1878.9(3)$ \\
\hline Z & 4 \\
\hline$\rho_{\text {calc }} \mathrm{g} / \mathrm{cm}^{3}$ & 1.241 \\
\hline$\mu / \mathrm{mm}^{-1}$ & 0.213 \\
\hline $\mathrm{F}(000)$ & 760.0 \\
\hline Crystal size $/ \mathrm{mm}^{3}$ & $0.41 \times 0.35 \times 0.05$ \\
\hline Radiation & $\operatorname{MoK} \alpha(\lambda=0.71073)$ \\
\hline \multicolumn{2}{|c|}{$2 \Theta$ range for data collection $/{ }^{\circ} 4.56$ to 49.994} \\
\hline Index ranges & $-22 \leq \mathrm{h} \leq 22,-12 \leq \mathrm{k} \leq 12,-11 \leq 1 \leq 11$ \\
\hline Reflections collected & 25776 \\
\hline Independent reflections & $3303\left[\mathrm{R}_{\text {int }}=0.0280, \mathrm{R}_{\text {sigma }}=0.0191\right]$ \\
\hline Data/restraints/parameters & $3303 / 51 / 221$ \\
\hline Goodness-of-fit on $\mathrm{F}^{2}$ & 1.068 \\
\hline Final $\mathrm{R}$ indexes $[\mathrm{I}>=2 \sigma(\mathrm{I})]$ & $\mathrm{R}_{1}=0.0541, \mathrm{wR}_{2}=0.1524$ \\
\hline Final $\mathrm{R}$ indexes [all data] & $\mathrm{R}_{1}=0.0625, \mathrm{wR}_{2}=0.1602$ \\
\hline Largest diff. peak/hole / e $\AA^{-}$ & $0.55 /-0.33$ \\
\hline
\end{tabular}


Figure S1. ORTEP plot of $59 \cdot \mathrm{HCl}$. Thermal ellipsoids drawn at $50 \%$ probability level.
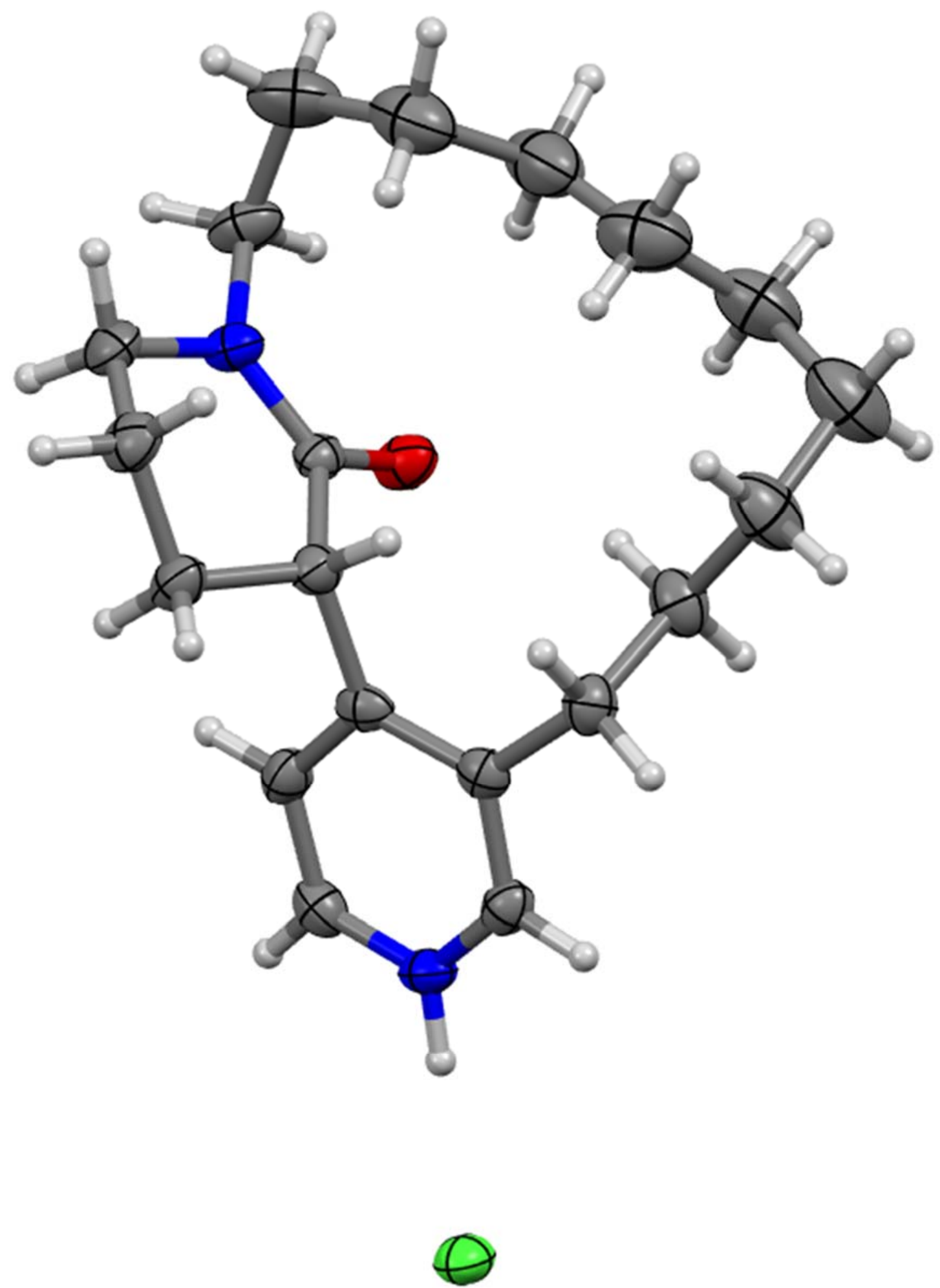


\section{NMR spectroscopic analysis of 61}

The final product $\mathbf{6 1}$ was characterized by a battery of one- and two-dimensional (1D and 2D)

homonuclear and ${ }^{1} \mathrm{H}-{ }^{13} \mathrm{C}$ heteronuclear experiments $\left[{ }^{1} \mathrm{H},{ }^{13} \mathrm{C}, 2 \mathrm{D}\right.$ correlated spectroscopy (COSY), total correlation spectroscopy (TOCSY) nuclear Overhauser effect spectroscopy (NOESY), heteronuclear single quantum coherence (HSQC) with spectral editing, and heteronuclear multiple bond correlation (HMBC)] using an NMR spectrometer operating at the proton frequency of $600 \mathrm{MHz}$. Gradient-assisted versions of the pulse sequences and inverse detection were used for these 2D experiments. HSQC with spectral editing allows the differentiation of $\mathrm{CH}_{2}$ from $\mathrm{CH} / \mathrm{CH}_{3}$ groups. ${ }^{1} \mathrm{H}$ spectral widths and $90^{\circ}$ pulse widths are optimized for each sample. A recycle delay (D1) of 4.0s was used in all the 2D experiments. An optimal range of mixing times for NOESY experiments was determined using the one dimensional version of spin-lattice relaxation measurements. Typical parameters for the NMR experiments were as follows: ${ }^{1} \mathrm{H}$ [time domain data points (TD), 32k; NS, 32], ${ }^{13} \mathrm{C}(\mathrm{TD}, 64 \mathrm{k}$; NS, 10k), 2D COSY (TD, 4k; TD1, 512; NS, 8; DS, 32), NOESY [TD, 4k; TD1, 300; NS, 16; DS, 64; mixing times, 0.05, 0.2, 0.5, 0.7, and 1.0s], ${ }^{13} \mathrm{C}-{ }^{1} \mathrm{H}$ HSQC (TD, 2k; TD1, 256; NS, 16; DS, 128) and ${ }^{13} \mathrm{C}-{ }^{1} \mathrm{H}$ HMBC (TD, 4k; TD1, 256; NS, 32; DS, 64). TD, NS, and DS refer to time domain data points, number of scans, and dummy scans, respectively. All NMR data were processed with TOPSPIN 3.0 suite of software programs. The $1 \mathrm{D}{ }^{1} \mathrm{H}$ data were processed with zero-filling to $64 \mathrm{k}$ data points and $0.2 \mathrm{~Hz}$ exponential line broadening, whereas ${ }^{13} \mathrm{C}$ spectra were processed with zero-filling to $128 \mathrm{k}$ data points and $1.0 \mathrm{~Hz}$ of exponential line broadening. The 2D NMR data were processed with the zero-filling to 4,096 points and 1,024 points in acquisition and second dimension, respectively. 


\section{Chemical shift table for compound 61}

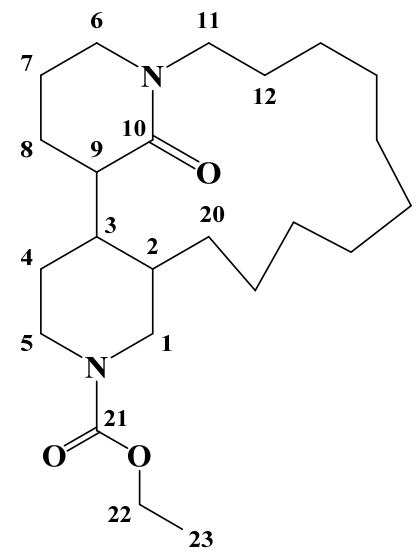

\begin{tabular}{|c|c|c|}
\hline Atom Number & ${ }^{1} \mathrm{H}$ & ${ }^{13} \mathrm{C}$ \\
\hline 1 & $2.77,4.16,4.29$ & 45.2 \\
\hline 2 & 2.58 & $39.4,39.9$ \\
\hline 3 & 2.48 & $46.4,46.7$ \\
\hline 4 & $1.63,1.72,1.84,1.94$ & $23.9,24.7$ \\
\hline 5 & $2.74,4.11,4.24$ & 47.6 \\
\hline 6 & $3.09,3.19,3.32,3.5$ & $47.4,50.6$ \\
\hline 7 & $1.62,1.92,1.95$ & 23.9 \\
\hline 8 & $2.43,2.56,4.46,4.52$ & $46.5,49.2$ \\
\hline 9 & 2.01 & $35.1,35.6$ \\
\hline 10 & - & $155.7,155.8,155.9,156.0$ \\
\hline 21 & - & $170.8,170.9$ \\
\hline 22 & $4.07,4.14$ & 61.2 \\
\hline 23 & 1.24 & $14.4,14.9$ \\
\hline
\end{tabular}




\section{NMR Analysis of Compound 61}

\section{Figure S2}

The overlayed ${ }^{1} \mathrm{H}-{ }^{1} \mathrm{H}$ COSY (A) and ${ }^{1} \mathrm{H}_{-}{ }^{13} \mathrm{C}$ HSQC (B) cross-sections of 61 . The data was collected at $278 \mathrm{~K}$ in $\mathrm{CDCl}_{3}$. The representative resonances and cross peaks are marked accordingly. The $\mathrm{CH}$ (black) and $\mathrm{CH}_{2}$ cross peaks (red) have opposite signs in the HSQC data, since the pulse sequence has an added multiplicity editing element. Note that $\mathrm{C}_{1} \mathrm{H}_{2}, \mathrm{C} 5 \mathrm{H}_{2}, \mathrm{C}_{6} \mathrm{H}_{2}$ and $\mathrm{C} 8 \mathrm{H}_{2}$ each has four sets of ${ }^{1} \mathrm{H}$ resonances and HSQC cross peaks, as a result of the chemical shift nonequivalence between the geminal protons and the presence of atropisomers/rotamers. Also, $\mathrm{H} 2-\mathrm{H} 9$, but not $\mathrm{H} 2-\mathrm{H} 3$, cross peak is observed in the COSY spectrum.

(A)
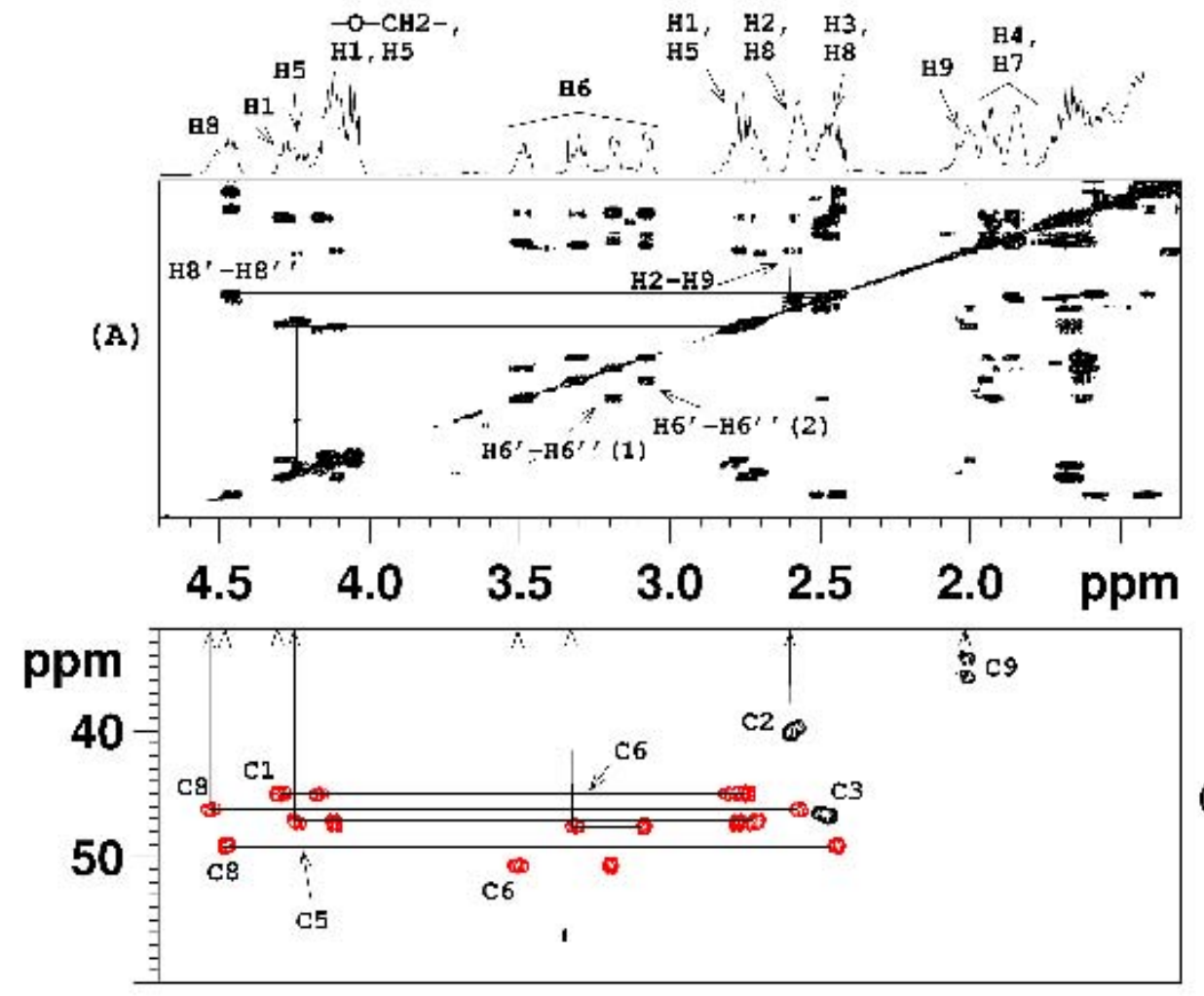

(B) 


\section{Figure S3}

The ${ }^{1} \mathrm{H}-{ }^{13} \mathrm{C}$ HMBC cross-sections of $\mathbf{6 1}$ showing the correlations of carbonyls to the protons of the piperidine and piperidone rings. The cross peaks between the carbonyls and the ring protons allowed the assignments, even though the proton resonances displayed severe overlap.

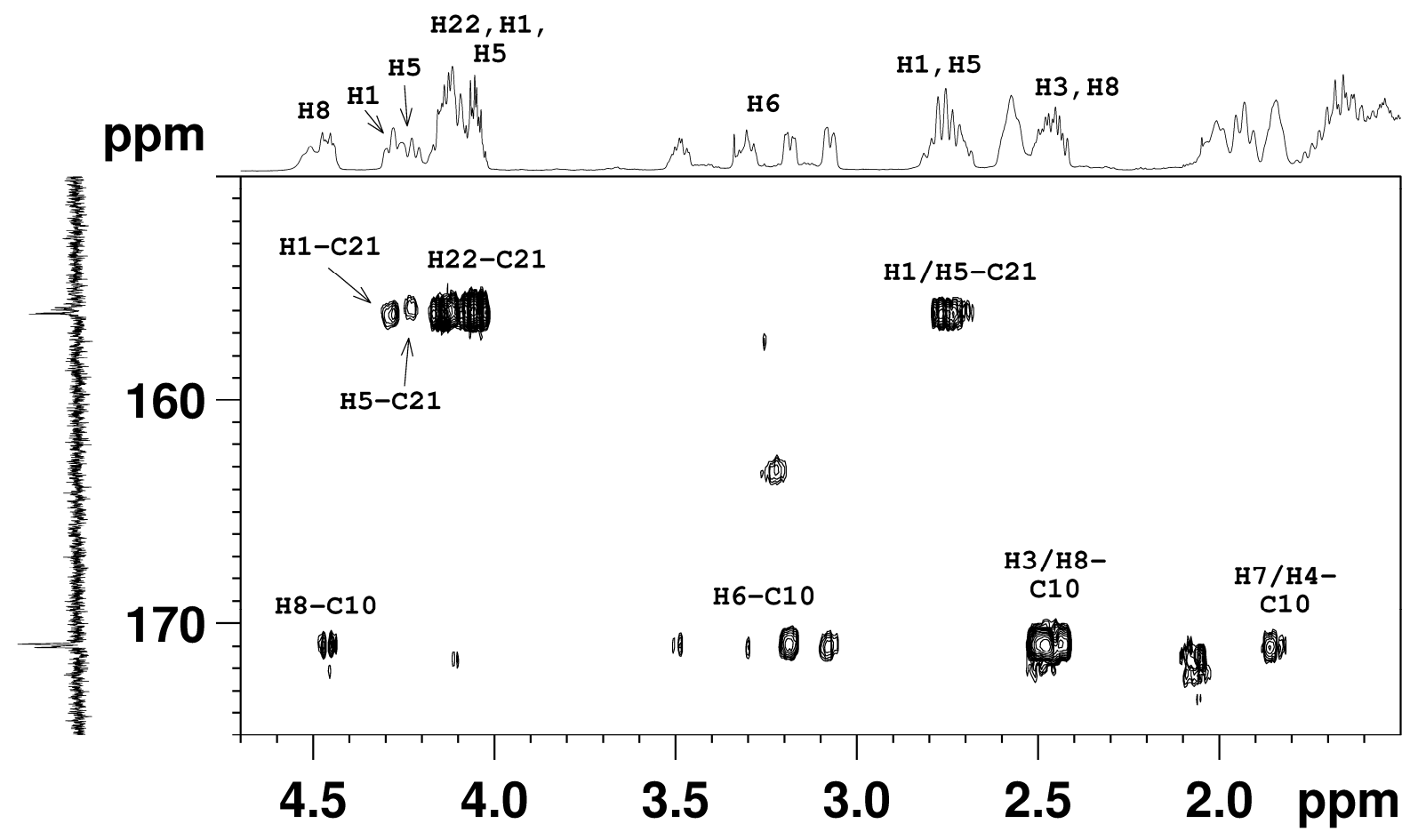




\section{Figure S4}

$\mathrm{A}^{1} \mathrm{H}^{-13} \mathrm{C}$ HSQC/HMBC overlayed cross-section of $\mathbf{6 1}$ showing the correlations of the bridging $\mathrm{CH}$ carbons of the piperidine and piperidone rings. HSQC cross peaks are colored red, whereas HMBC peaks are colored black.

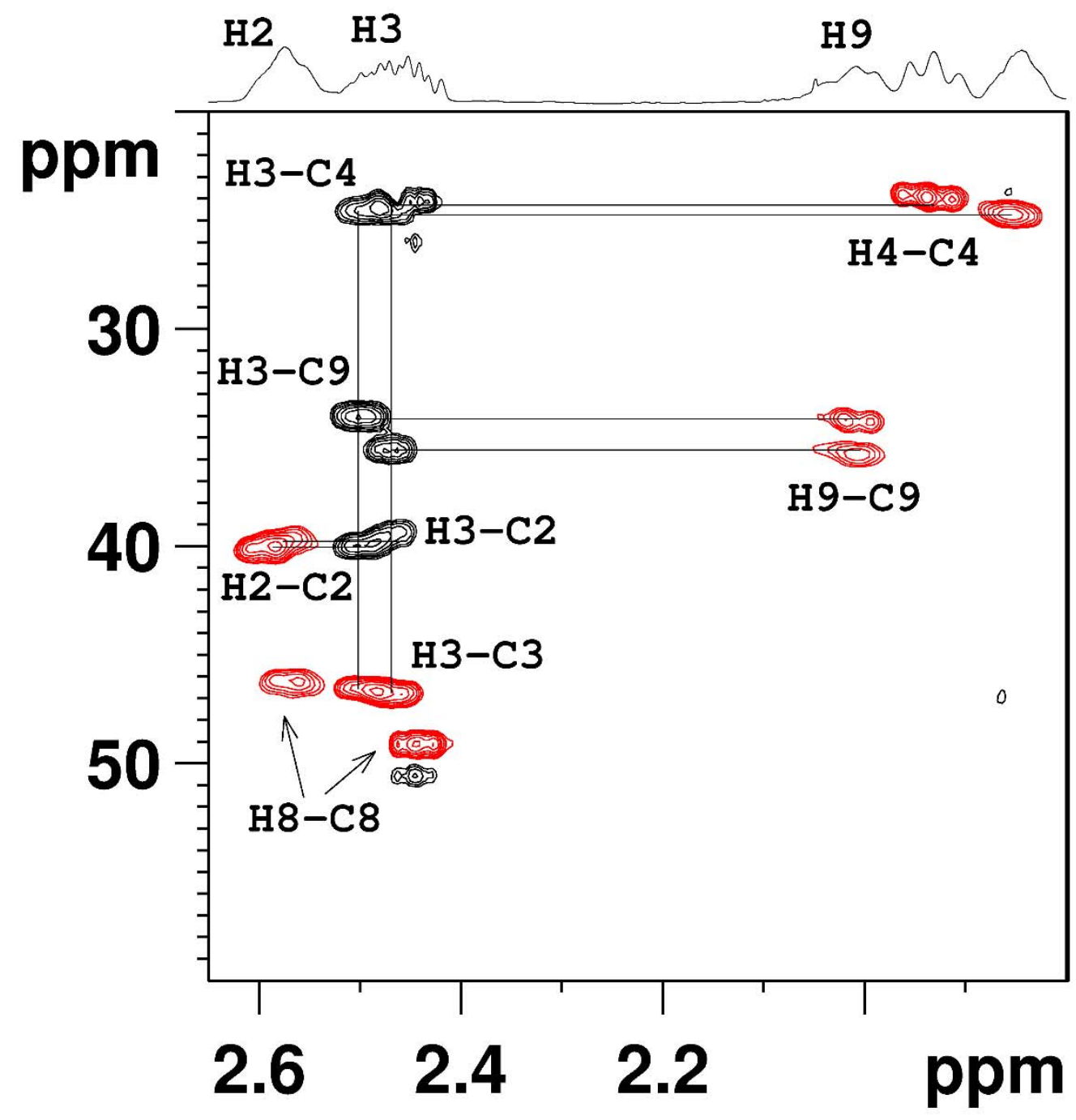




\section{Figure S5}

A NOESY (mixing time $=0.7 \mathrm{~s}$ ) cross-section of $\mathbf{6 1}$ showing the unique NOE's supporting the relative cis stereochemistry of $\mathrm{H} 9, \mathrm{H} 2$ and $\mathrm{H} 3$. The semiquantitative analysis of the mixing time dependent NOEs of $\mathrm{H} 2-\mathrm{H} 3$ and $\mathrm{H} 6$ '-H6' gives a distance of $2.52 \AA$ between $\mathrm{H} 2$ and $\mathrm{H} 3 ; \mathrm{r}_{\mathrm{H} 2-\mathrm{H} 3}($ cis $)$ and $\mathrm{r}_{\mathrm{H} 2-\mathrm{H} 3}$ (anti) are expected to be 2.50 and $3.05 \AA$ respectively. A strong NOE was observed between $\mathrm{H} 9$ and $\mathrm{H} 2$, but quantitative NOE analysis was less reliable due to resonance overlap and ${ }^{13} \mathrm{C}$-edited NOE measurements are necessary for such an analysis.

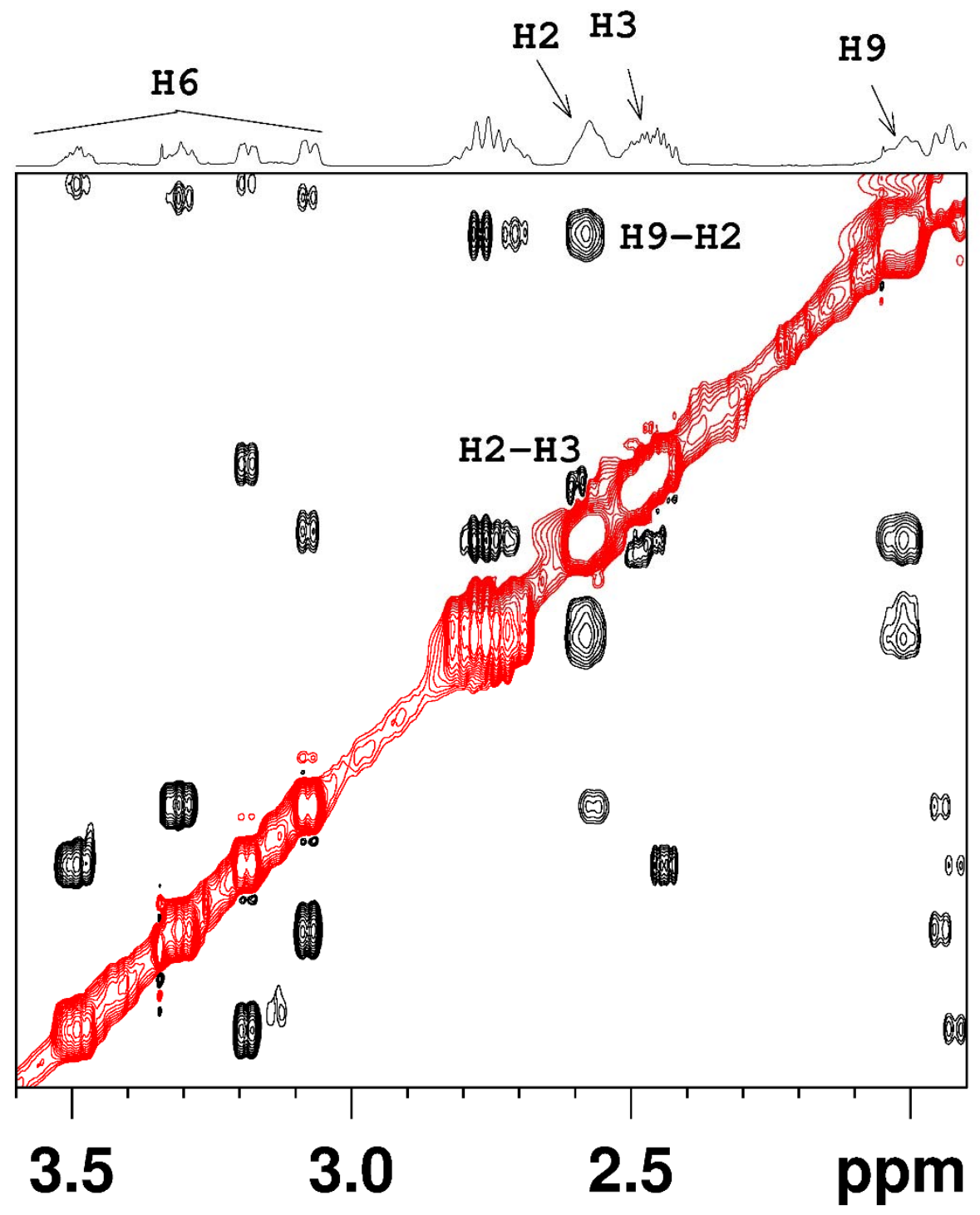




\section{Semiquantitative NOE analysis}

The NOE between $\mathrm{H} 2$ and $\mathrm{H} 3$ was analyzed semi-quantitatively using reference distance method. First, the mixing time dependent NOE cross peak volumes were fitted to either linear or $2^{\text {nd }}$ order polynomial equation (Equation 1) and the best fit was chosen to estimate the ${ }^{1} \mathrm{H}-{ }^{1} \mathrm{H}$ cross relaxation constants.

$\operatorname{NOE}_{\mathrm{ij}}\left(\tau_{\mathrm{m}}\right)=\mathrm{B}_{0}-\mathrm{B}_{1} \tau_{\mathrm{m}}+\mathrm{B}_{2} \tau_{\mathrm{m}}^{2}-\mathrm{B}_{3} \tau_{\mathrm{m}}^{3}+\ldots \ldots$

$\mathrm{B}_{1}$ and $\tau_{\mathrm{m}}$ are the cross relaxation constant $\left(\sigma_{\mathrm{ij}}\right)$ and the mixing time, respectively. $\mathrm{B}_{2}, \mathrm{~B}_{3} \ldots$ are product functions of the cross relaxation constants. $i$ and $j$ denote the protons generating the NOE correlation. Note that the linear form is Equation 1 truncated to first order in $\tau_{\mathrm{m}}$. The unknown internuclear distance was estimated from the cross relaxation constants by using the following equation:

$\mathrm{r}_{\mathrm{ij}}^{6}=\mathrm{r}_{\mathrm{ref}}^{6}\left(\sigma_{\mathrm{ref}} / \sigma_{\mathrm{ij}}\right)$

$r_{i j}$ and $r_{r e f}$ designate unknown and reference internuclear distances, whereas $\sigma_{\text {ref }}$ refers to the cross relaxation constant of the reference proton pair. The reference proton pair and the reference distance used in the calculations are $\mathrm{H}_{6}, \mathrm{H}_{6}$, and $1.75 \AA$ respectively. The NOE buildup curves of H6'-H6' ' and H2-H3 are shown below: 

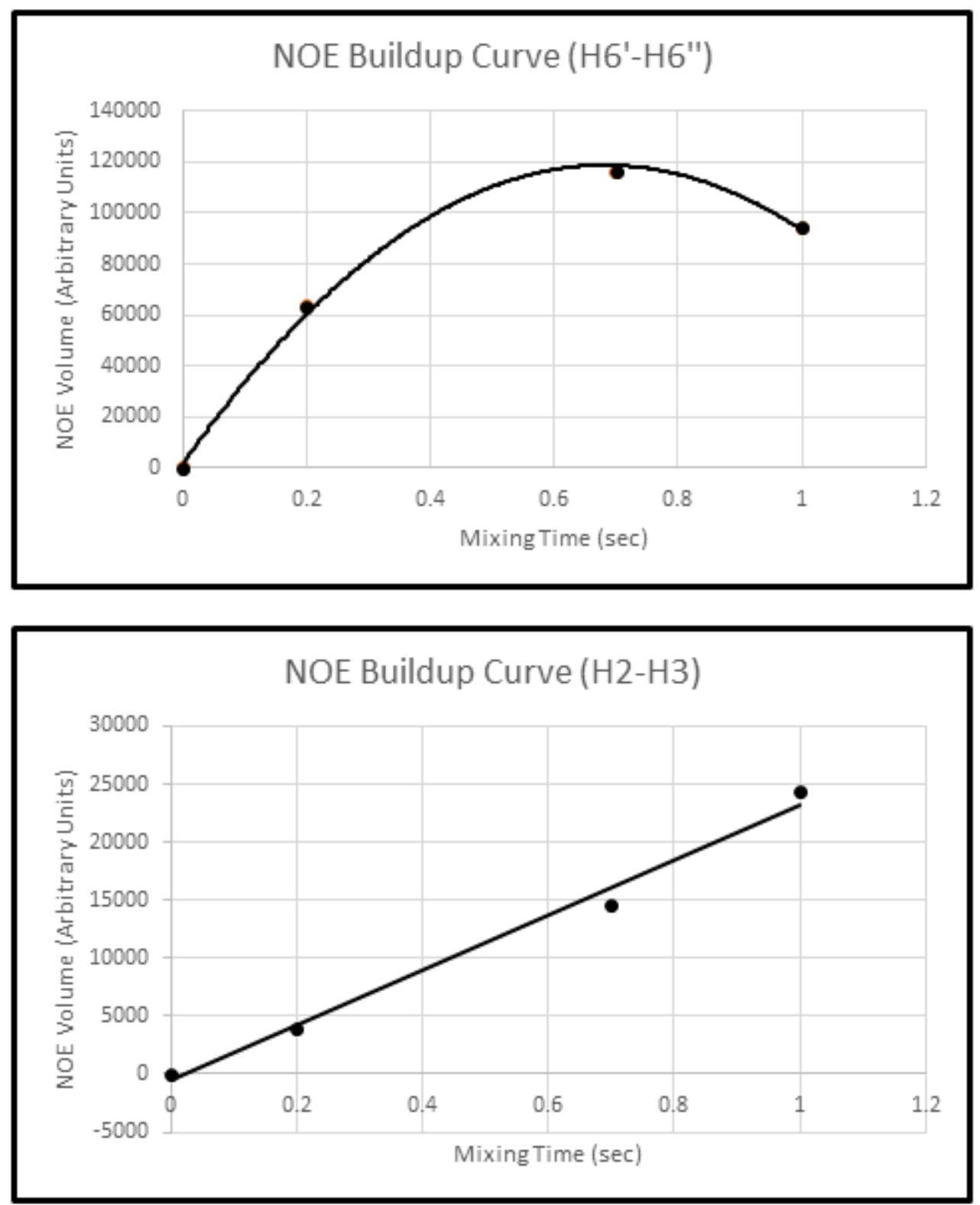


\section{Coordinates for the Optimized lowest energy conformation of 61}

Level of theory $=$ DFT geom opt b3lyp/6-31g(d)

Total Energy $=-1235.75289 \mathrm{au}$

RMS Displacement $=0.000174$

Dipole Moment $=2.9576$ Debye

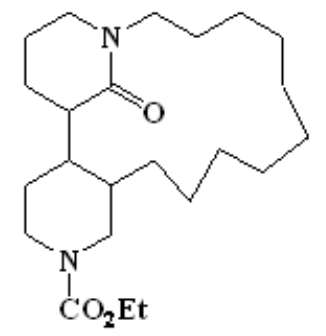

\begin{tabular}{lrrr} 
Symbol & $\mathbf{X}$ & $\mathbf{Y}$ & $\mathbf{Z}$ \\
\hline $\mathrm{C}$ & 1.82005800 & 3.37993400 & -0.68618100 \\
$\mathrm{~N}$ & 2.22505300 & 2.19888300 & 0.08043800 \\
$\mathrm{C}$ & 0.97224700 & 4.30185700 & 0.18891600 \\
$\mathrm{C}$ & -0.30100900 & 3.57813000 & 0.63947400 \\
$\mathrm{C}$ & -0.03368100 & 2.13065200 & 1.15525100 \\
$\mathrm{C}$ & 1.42761500 & 1.66832300 & 1.06310800 \\
$\mathrm{O}$ & 1.84342000 & 0.82176200 & 1.85649400 \\
$\mathrm{C}$ & -1.05835000 & 1.11594900 & 0.54192300 \\
$\mathrm{C}$ & -1.04762200 & 1.00250900 & -0.99933300 \\
$\mathrm{C}$ & -1.93867300 & -0.16961600 & -1.48368300 \\
$\mathrm{~N}$ & -2.93933500 & -0.54442000 & -0.48457600 \\
$\mathrm{C}$ & -2.49566600 & -0.88222700 & 0.87930500 \\
$\mathrm{C}$ & -1.08717400 & -0.30979400 & 1.16363900 \\
$\mathrm{C}$ & 3.57435700 & 1.65919400 & -0.10362300 \\
$\mathrm{C}$ & 3.73403200 & 0.83733100 & -1.39581900 \\
$\mathrm{C}$ & 5.00872400 & -0.03121800 & -1.39694700 \\
$\mathrm{C}$ & 4.89456000 & -1.31665400 & -0.55119800 \\
$\mathrm{C}$ & 4.04775900 & -2.41492500 & -1.22217000 \\
$\mathrm{C}$ & 3.65486500 & -3.61796800 & -0.32743100 \\
$\mathrm{C}$ & 2.25147900 & -3.51921200 & 0.30772800 \\
$\mathrm{C}$ & 2.13263500 & -2.52994300 & 1.48009500 \\
$\mathrm{C}$ & 0.70271300 & -2.08582000 & 1.83400500 \\
$\mathrm{C}$ & 0.04219100 & -1.27523000 & 0.69953500 \\
$\mathrm{C}$ & -4.24256700 & -0.66169300 & -0.87627200
\end{tabular}




\begin{tabular}{|c|c|c|c|}
\hline $\mathrm{O}$ & -4.67116100 & -0.41909000 & -1.99608100 \\
\hline $\mathrm{O}$ & -5.02477700 & -1.09231100 & 0.15522000 \\
\hline $\mathrm{C}$ & -6.43190200 & -1.21556400 & -0.13652900 \\
\hline $\mathrm{C}$ & -7.15666200 & 0.11317400 & 0.02073300 \\
\hline $\mathrm{H}$ & 2.73374700 & 3.88952200 & -1.01208400 \\
\hline $\mathrm{H}$ & 1.57338500 & 4.60696800 & 1.05533500 \\
\hline $\mathrm{H}$ & 0.71652400 & 5.21247500 & -0.36604500 \\
\hline 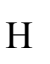 & -0.99561200 & 3.54967800 & -0.20661800 \\
\hline 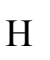 & -0.80336500 & 4.15681700 & 1.42287300 \\
\hline $\mathrm{H}$ & -0.21872400 & 2.10522300 & 2.23462000 \\
\hline$T$ & -0.02782100 & 0.84570800 & -1.37077500 \\
\hline H & -1.40470600 & 1.93624300 & -1.44609700 \\
\hline $\mathrm{H}$ & -1.31652100 & -1.04100500 & -1.72261900 \\
\hline$H$ & -2.48544800 & 0.09349700 & -2.38867300 \\
\hline$U$ & -2.50330900 & -1.97106400 & 1.02968700 \\
\hline$H$ & -3.21953900 & -0.45739300 & 1.58023500 \\
\hline $\mathrm{H}$ & 4.29135100 & 2.49200100 & -0.09934700 \\
\hline $\mathrm{H}$ & 3.77569800 & 1.04200900 & 0.77243700 \\
\hline$H$ & 2.84964400 & 0.19903400 & -1.51439900 \\
\hline $\mathrm{H}$ & 3.74276900 & 1.51313500 & -2.26157000 \\
\hline $\mathrm{H}$ & 5.25731200 & -0.30638600 & -2.43112800 \\
\hline $\mathrm{H}$ & 5.85304700 & 0.57362700 & -1.03618500 \\
\hline $\mathrm{H}$ & 5.90101000 & -1.71603600 & -0.36543200 \\
\hline $\mathrm{H}$ & 4.48748000 & -1.07052000 & 0.43603500 \\
\hline $\mathrm{H}$ & 3.12826200 & -1.97597900 & -1.63422700 \\
\hline $\mathrm{H}$ & 4.61117100 & -2.77966100 & -2.09222900 \\
\hline Н & 3.68088800 & -4.52784800 & -0.94108800 \\
\hline $\mathrm{H}$ & 4.41195200 & -3.76541400 & 0.45681700 \\
\hline $\mathrm{H}$ & 1.54373400 & -3.24852100 & -0.48826900 \\
\hline$\Pi$ & 1.94163700 & -4.51592100 & 0.65310500 \\
\hline $\mathrm{H}$ & 2.60873000 & -2.97434900 & 2.36567200 \\
\hline
\end{tabular}




$\begin{array}{lrrr}\mathrm{H} & 2.69242100 & -1.61441300 & 1.26526300 \\ \mathrm{H} & 0.07827200 & -2.95227500 & 2.09902900 \\ \mathrm{H} & 0.76673800 & -1.45246800 & 2.72510900 \\ \mathrm{H} & 0.82994500 & -0.71046500 & 0.19522100 \\ \mathrm{H} & -0.35627800 & -1.96876200 & -0.05050100 \\ \mathrm{H} & -6.79234800 & -1.95380900 & 0.58521900 \\ \mathrm{H} & -6.55059100 & -1.60976700 & -1.14851000 \\ \mathrm{H} & -7.00401300 & 0.52454600 & 1.02439000 \\ \mathrm{H} & -8.23317500 & -0.02676800 & -0.13333000 \\ \mathrm{H} & -6.79429000 & 0.83319500 & -0.71826800 \\ \mathrm{H} & 1.26868900 & 3.09993600 & -1.59598900 \\ \mathrm{H} & -1.00863900 & -0.20325500 & 2.25222200 \\ \mathrm{H} & -2.02948800 & 1.56121900 & 0.80686100\end{array}$


AL-05-16.1.fid

1H AL-05-16

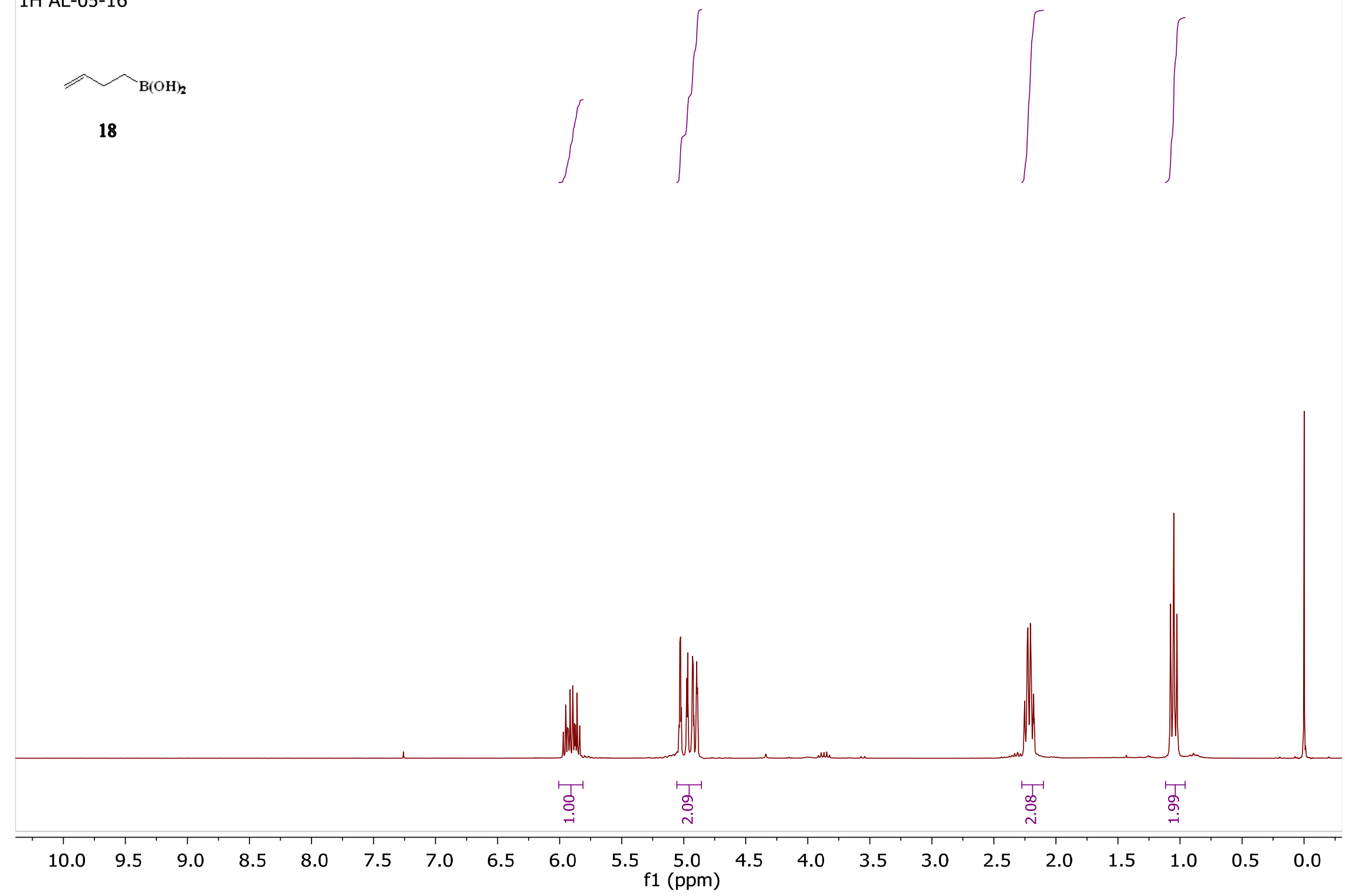


AL-05-16.2.fid

13C AL-05-16

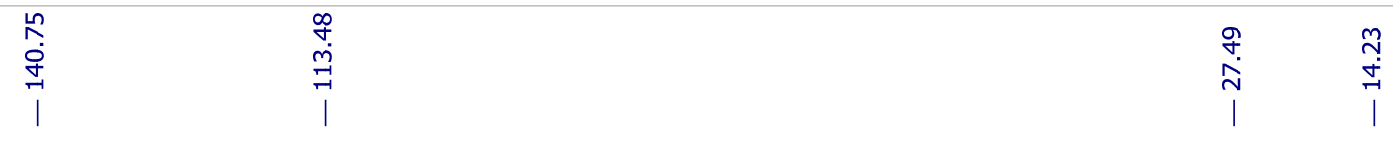

$\overbrace{\mathrm{B}(\mathrm{OH})_{2}}$

18

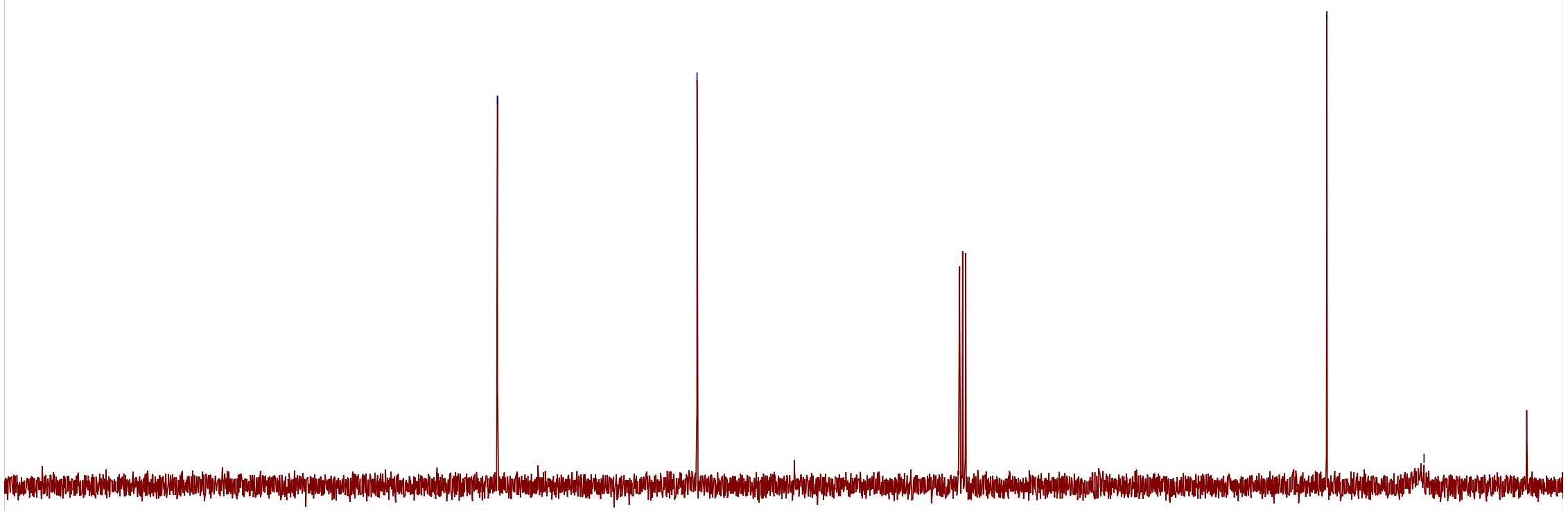

200

$190 \quad 180 \quad 170$

160

150

$140 \quad 130$

120

$\begin{array}{ll}110 & 100 \\ \mathrm{f} 1 & (\mathrm{ppm})\end{array}$

80

$70 \quad 60$

$60 \quad 50$

50

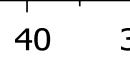

$30 \quad 20 \quad 10 \quad 0$


AL-05-24.1.fid

1H AL-05-24

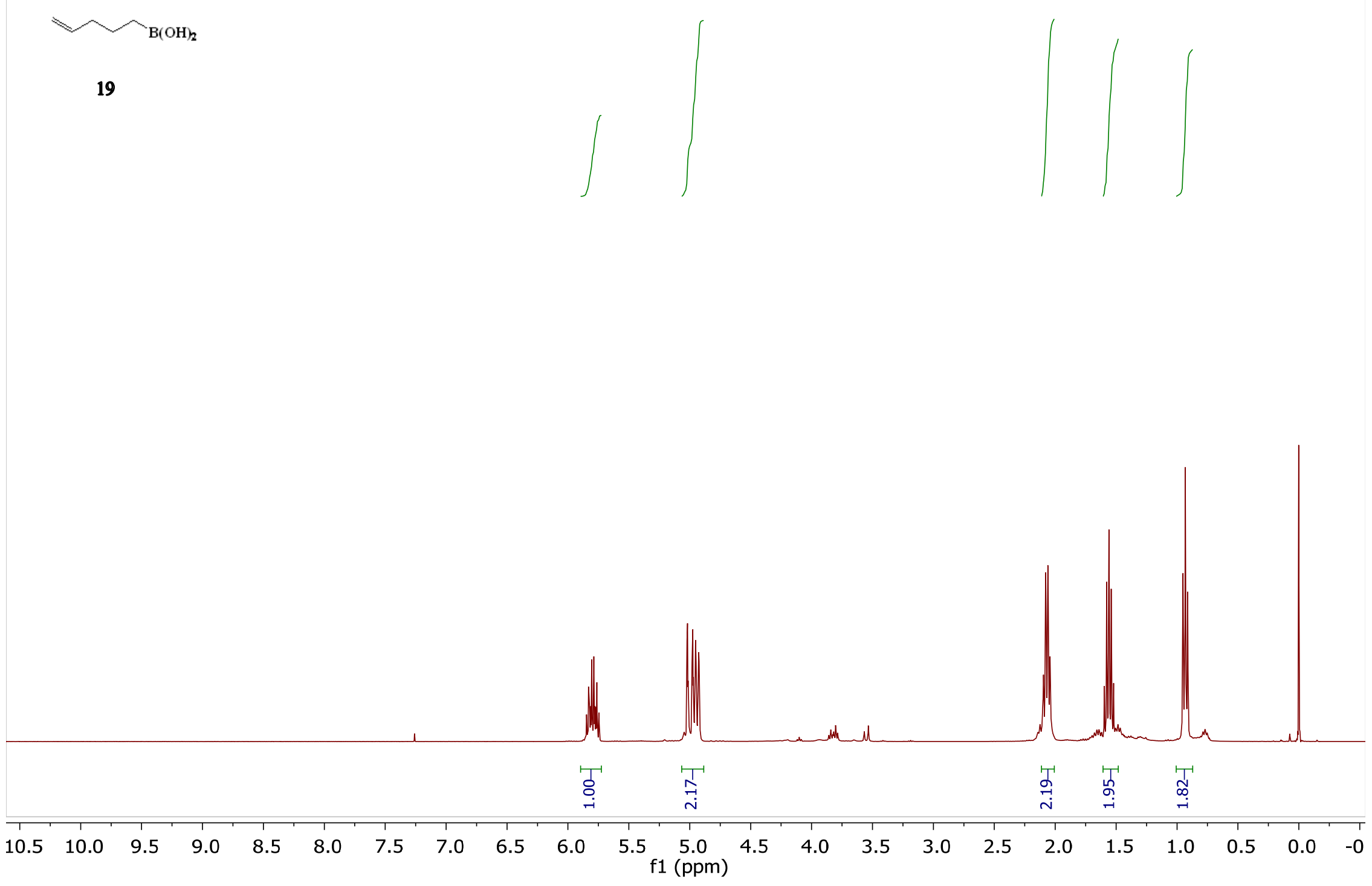




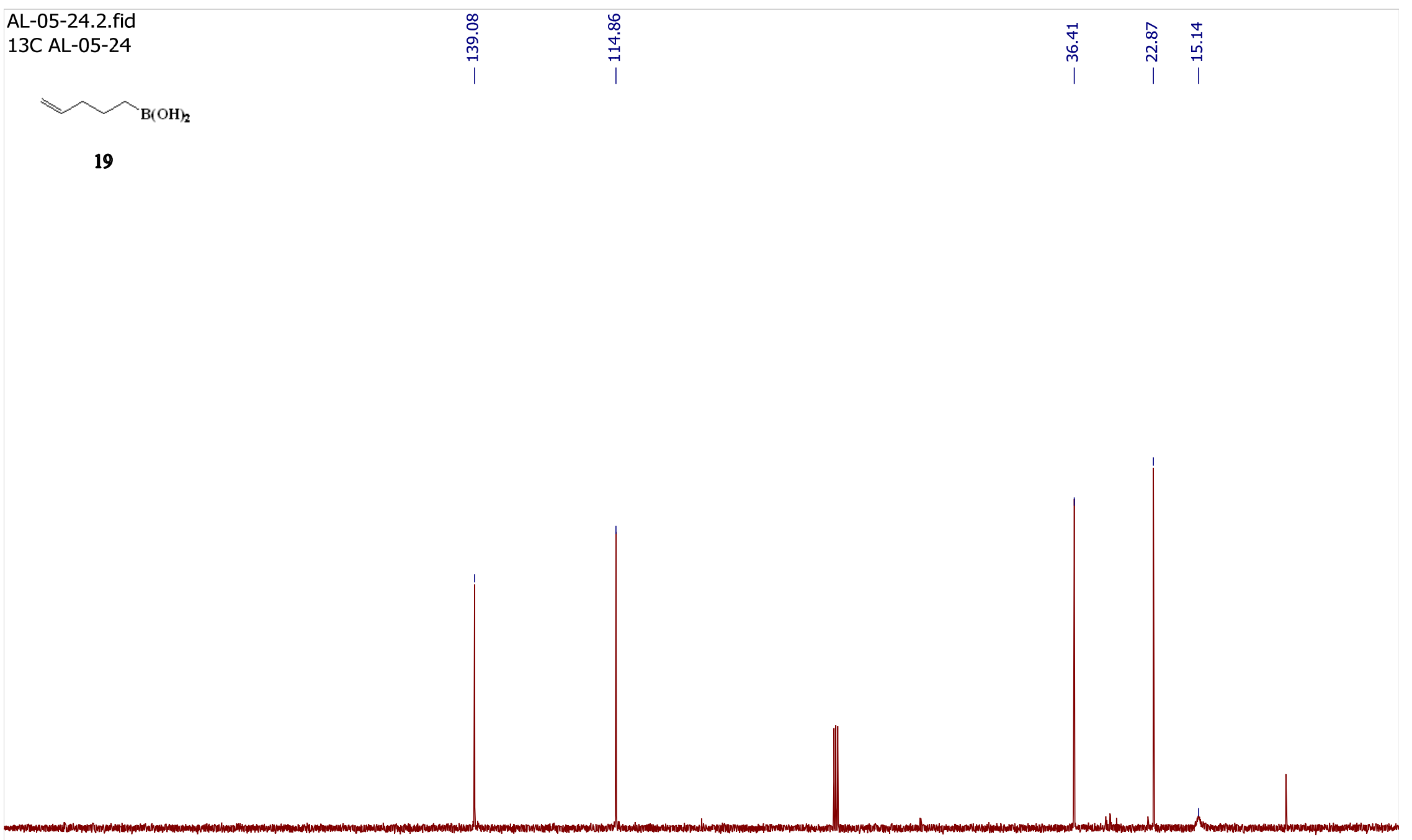

$\begin{array}{llllllllllllllllllllllllllllllllll}210 & 200 & 190 & 180 & 170 & 160 & 150 & 140 & 130 & 120 & 110 & \begin{array}{l}100 \\ \mathrm{f} 1\end{array} & 90 & 80 & 70 & 60 & 50 & 40 & 30 & 20 & 10 & 0 & -10 & \end{array}$


AL-05-32.1.fid

1H AL-05-32

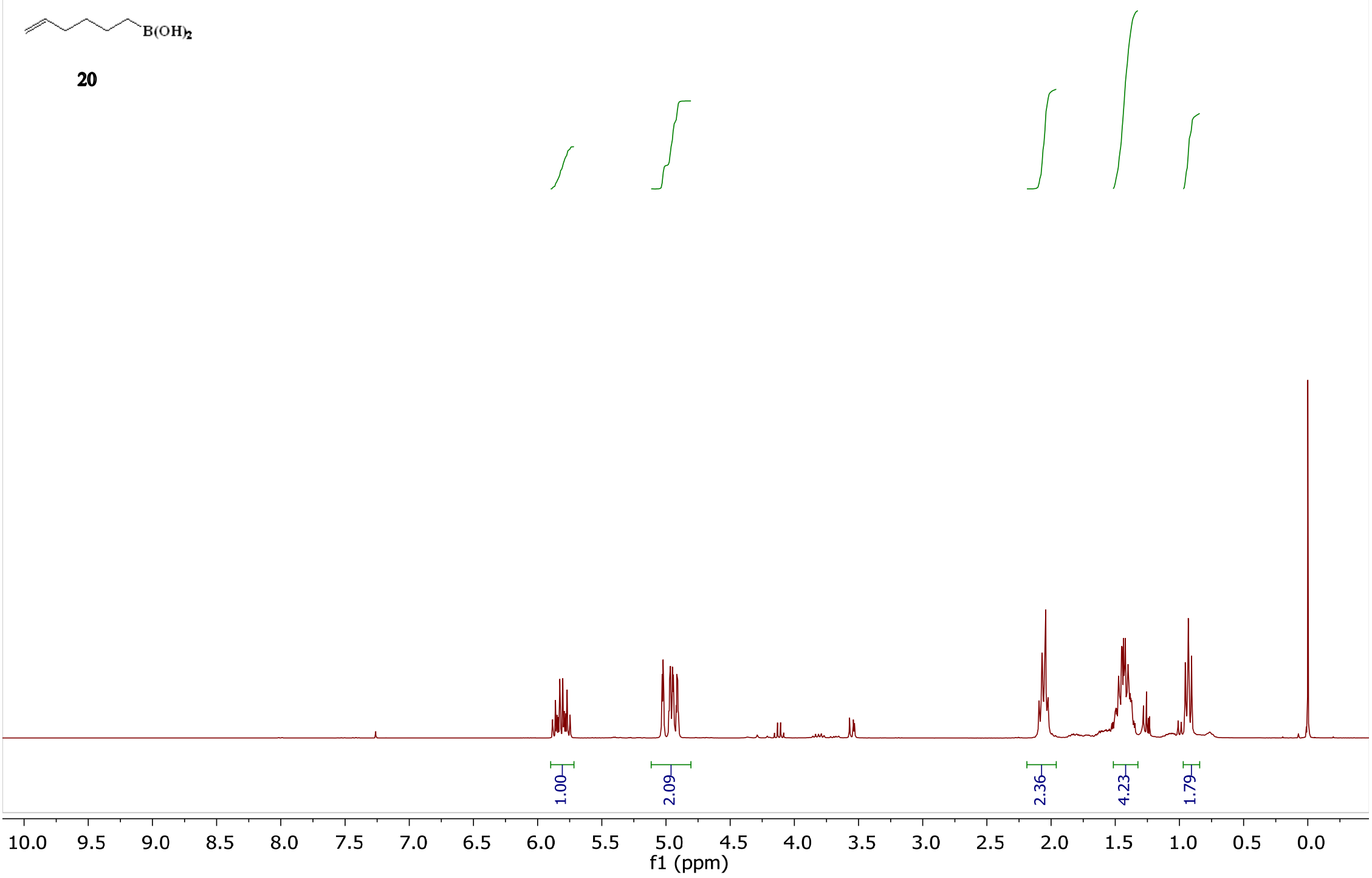


AL-05-32.2.fid

13C AL-05-32

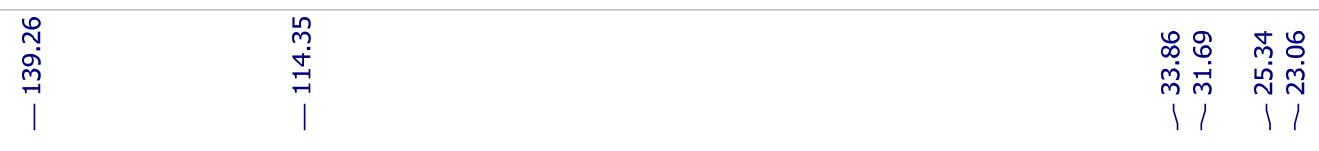

$\curvearrowright_{\mathrm{B}(\mathrm{OH})_{2}}$

20

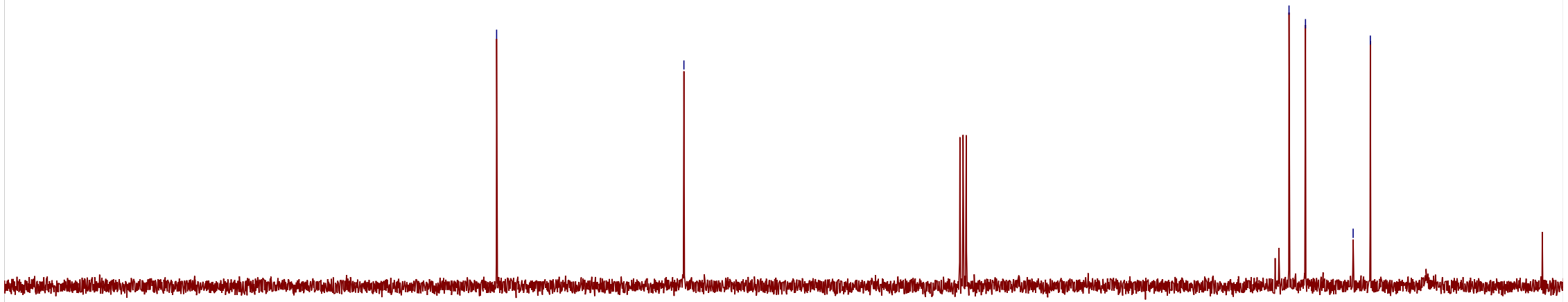

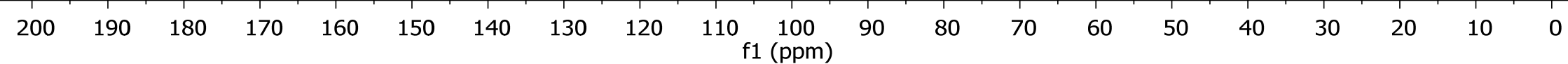


AL-05-22.1.fid

$1 \mathrm{H}$ AL-05-22

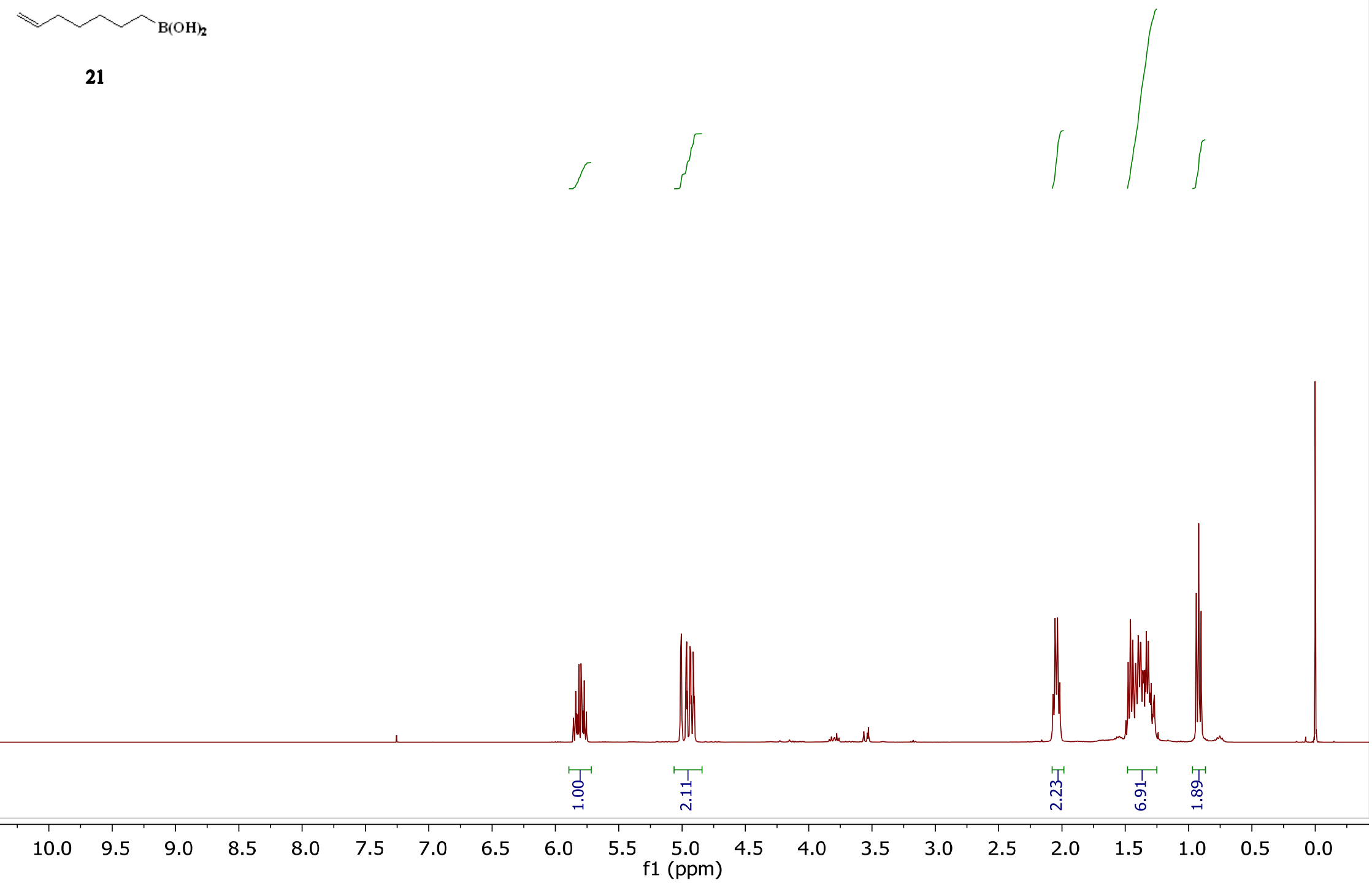




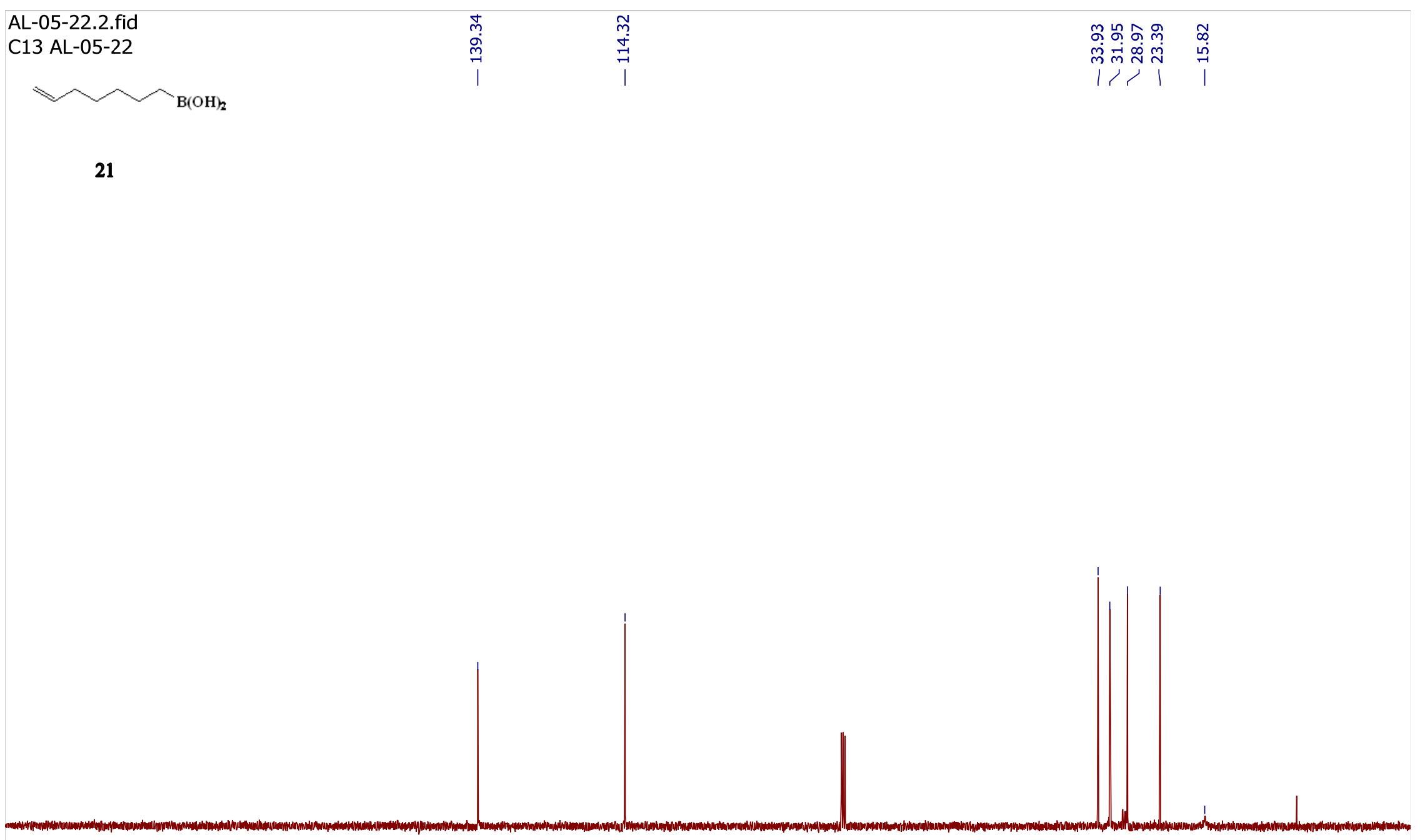

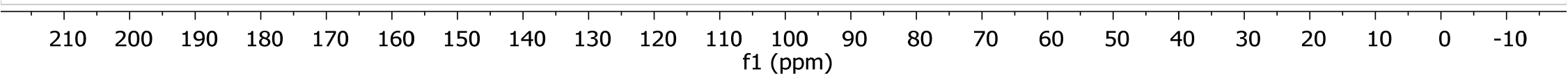


AL-05-36.1.fid

1H AL-05-36
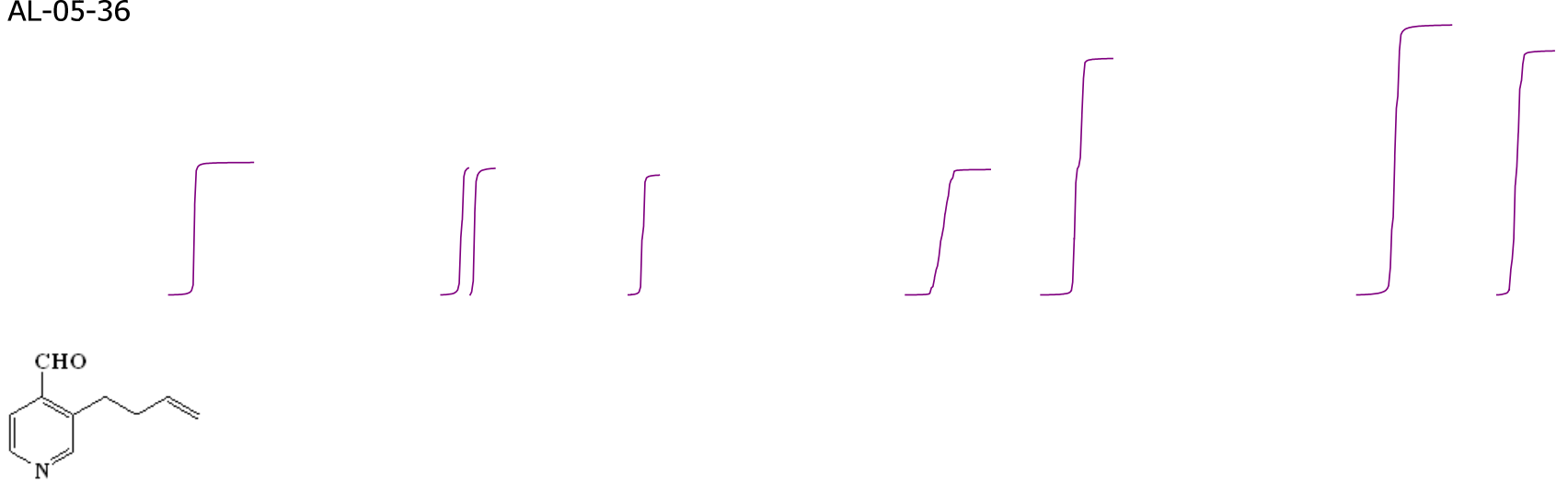

22

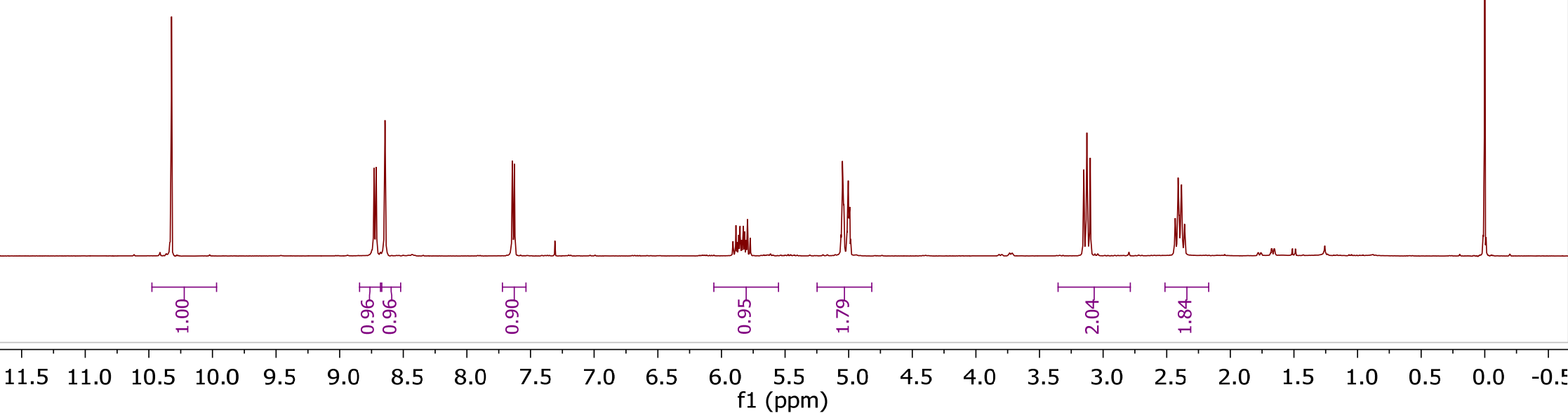


AL-05-362. fid

13C AL-05ं-36

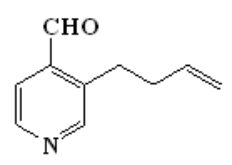

22

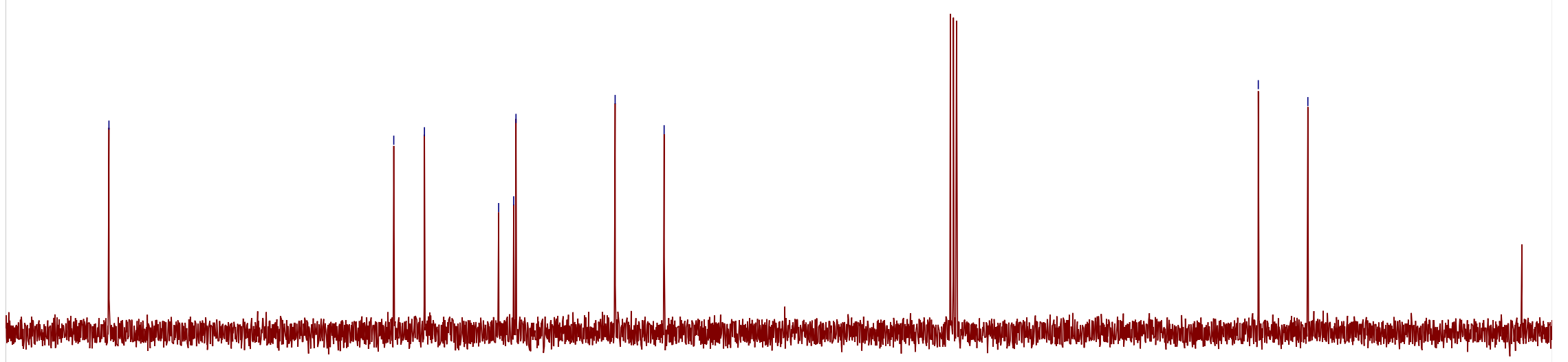

200 190

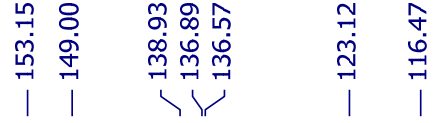

के
ஸे 
AL-04-270.1.fid

1H AL-04-270
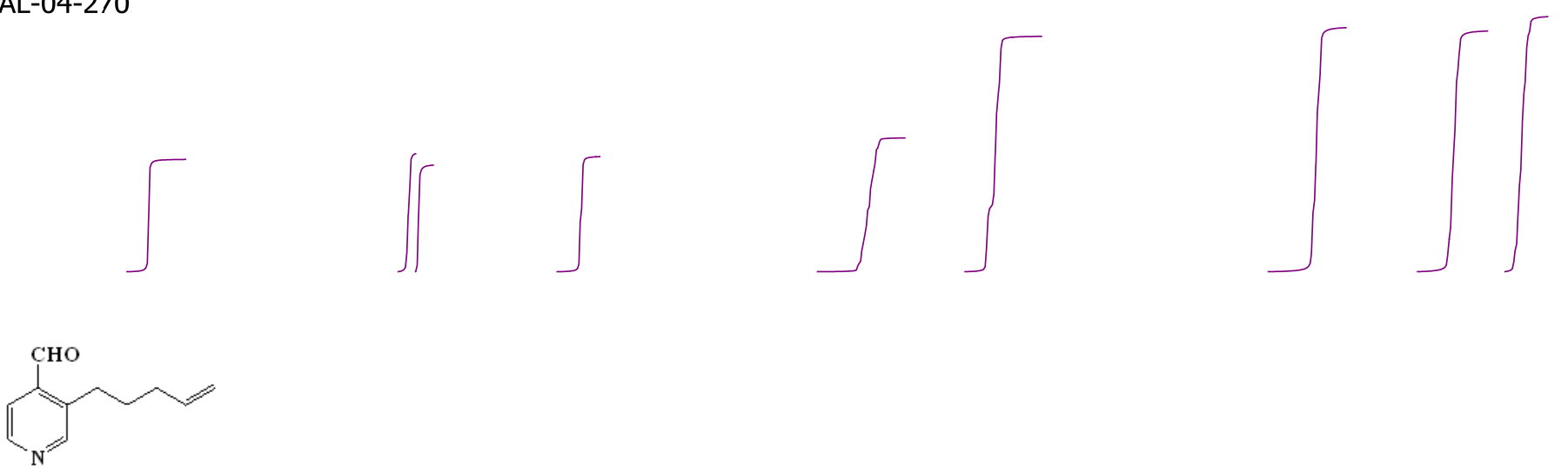

23

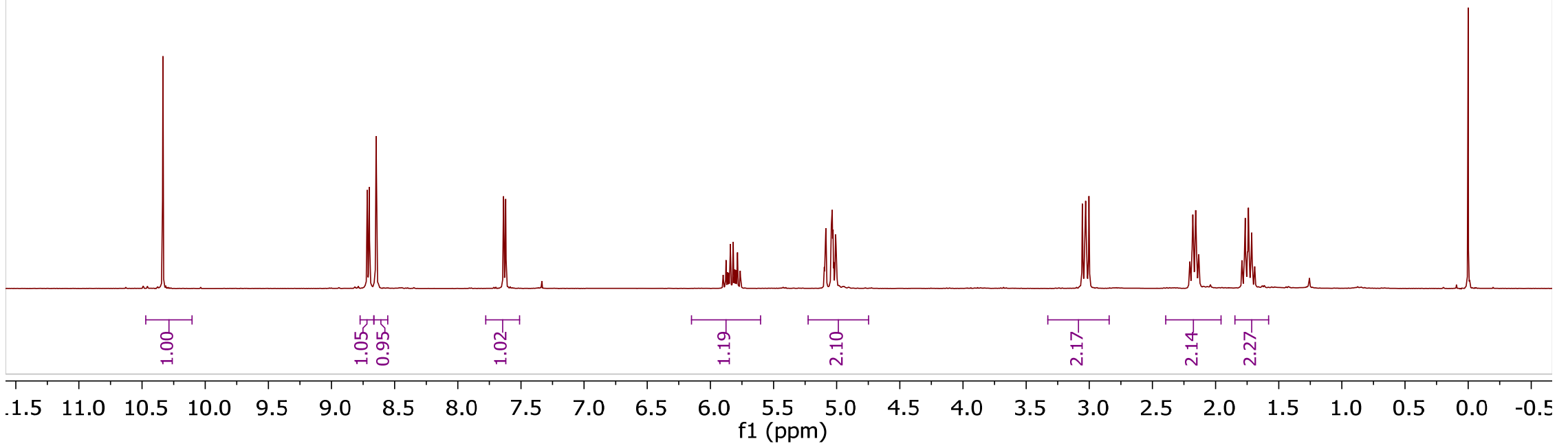




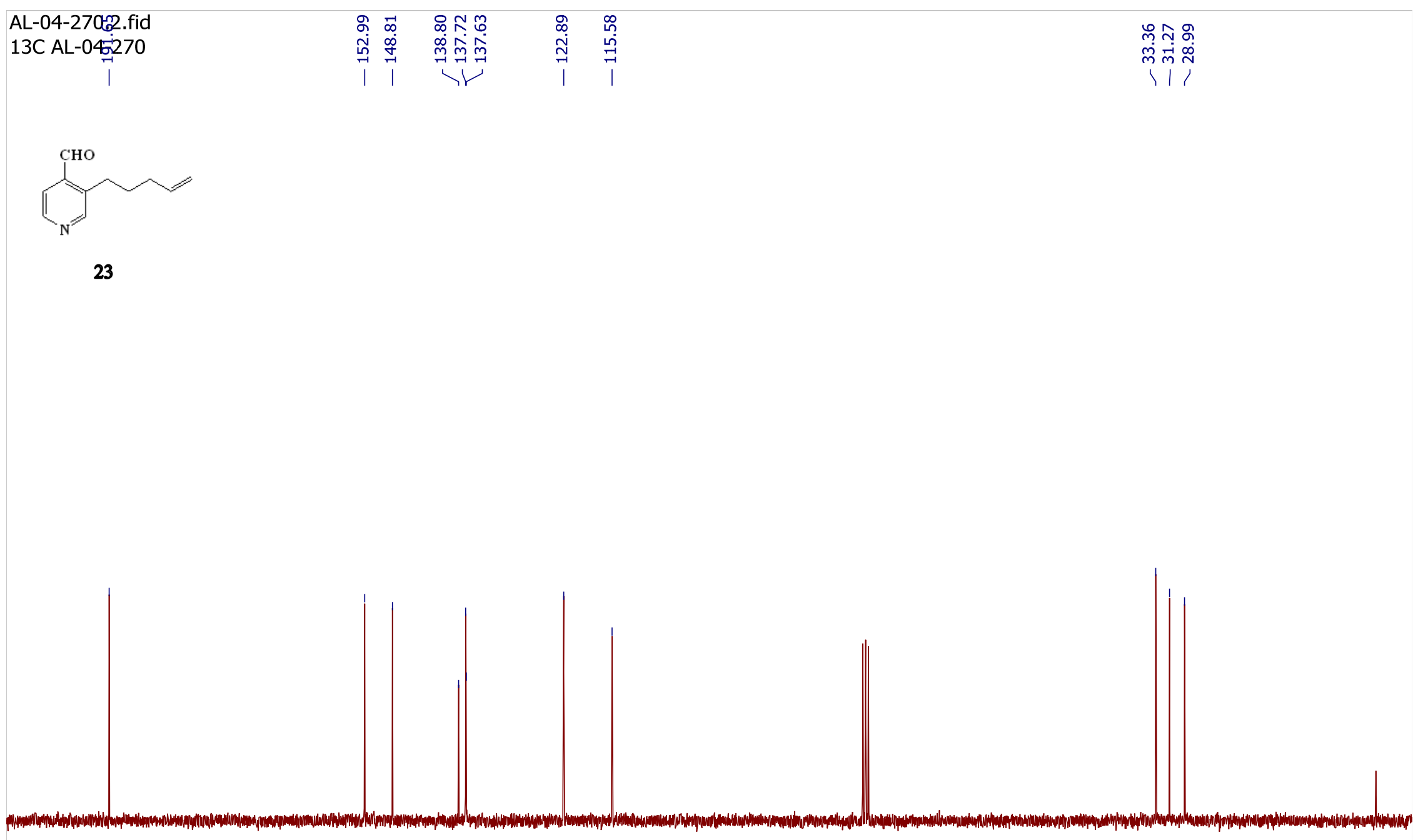

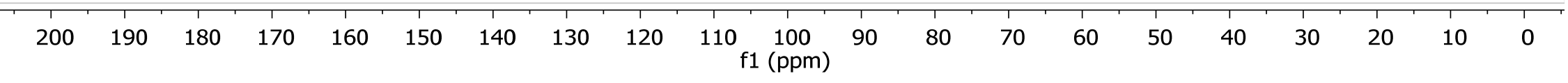


AL-05-48.1.fid

1H AL-05-48
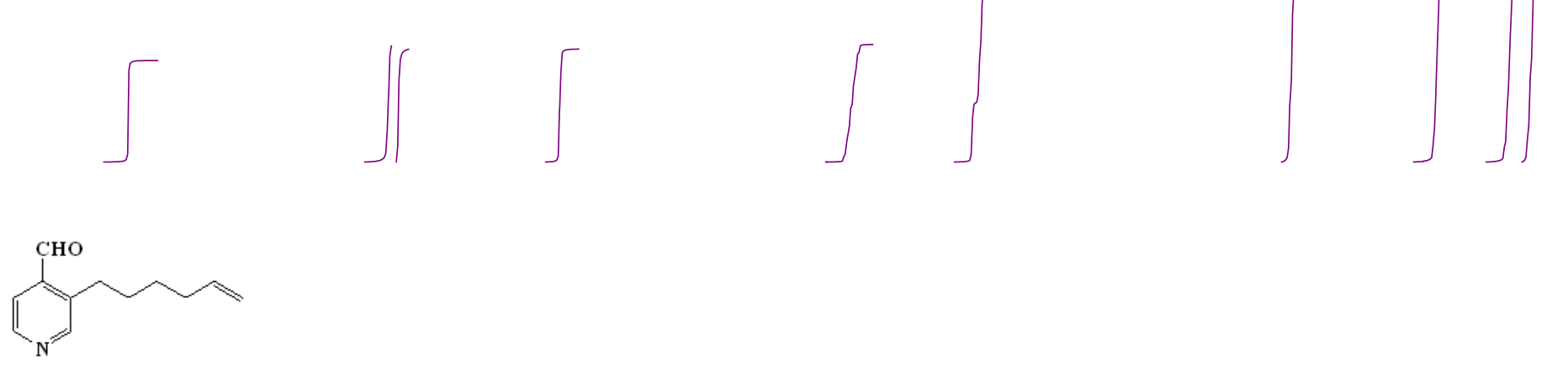

24

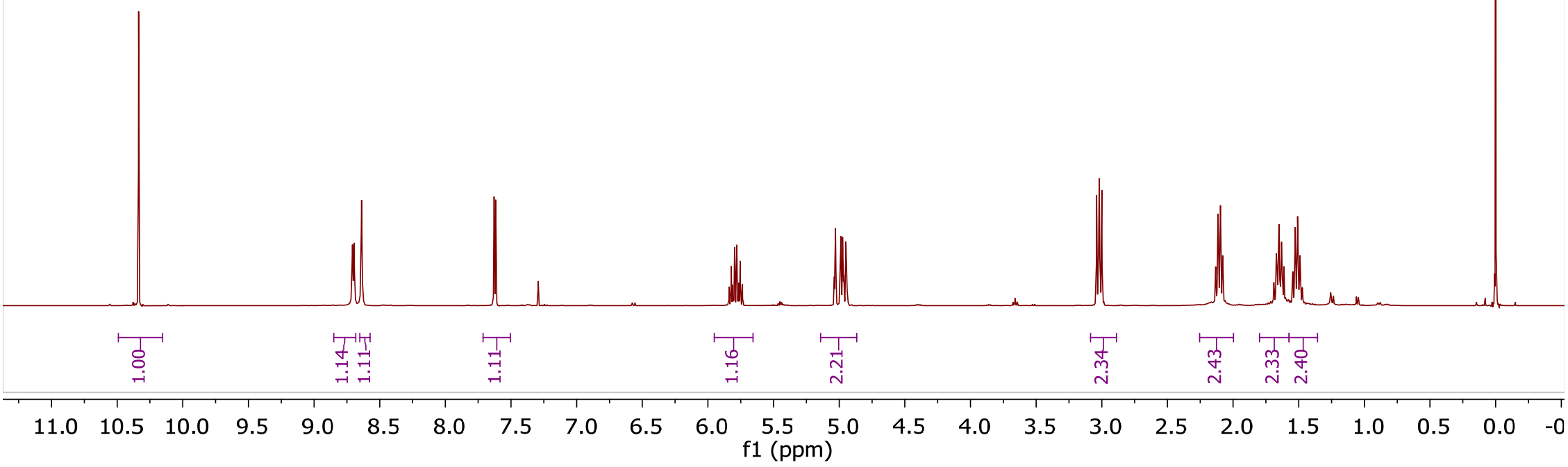




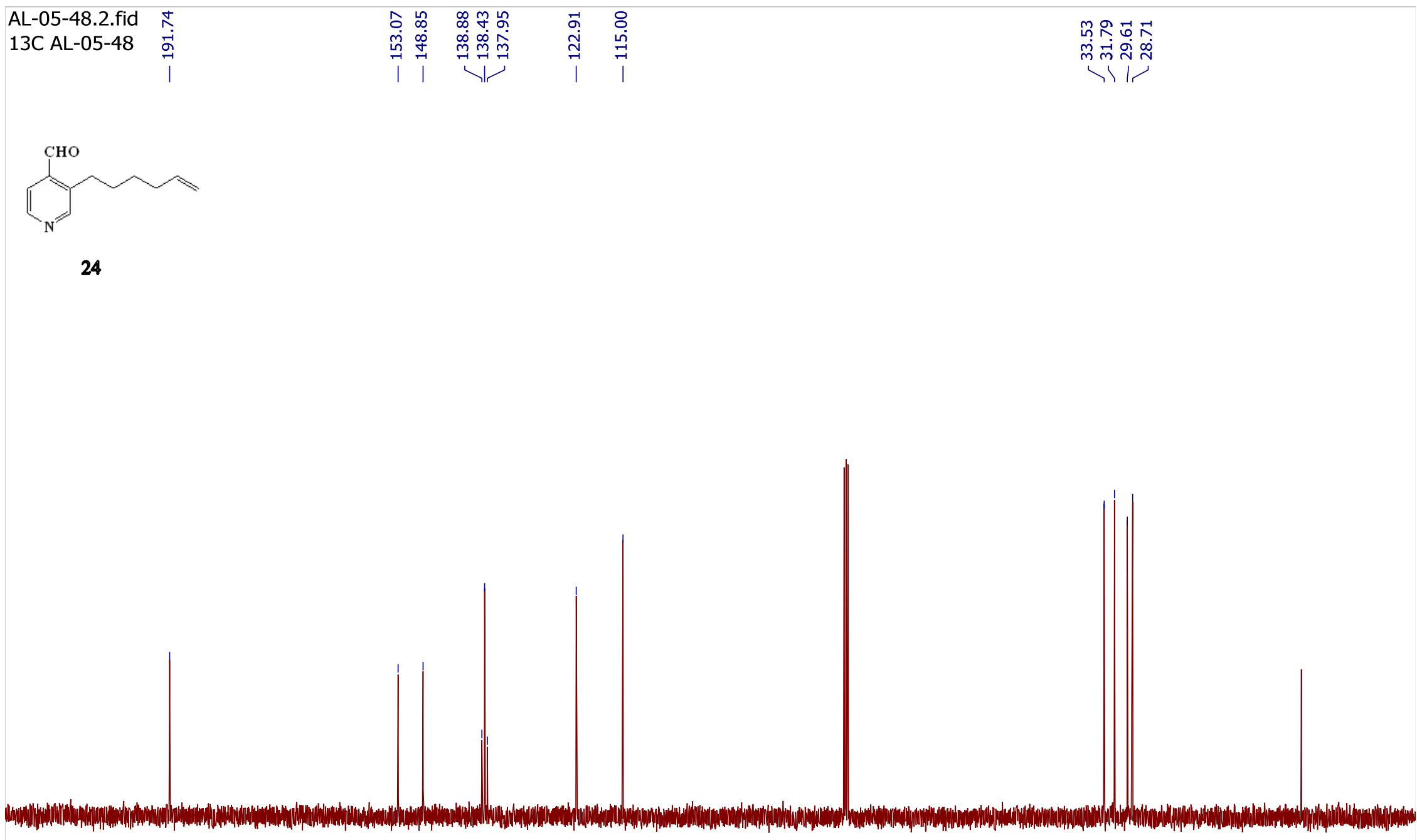

$\begin{array}{llllllllllllllllllllllll}210 & 200 & 190 & 180 & 170 & 160 & 150 & 140 & 130 & 120 & 110 & \begin{array}{l}100 \\ \mathrm{f} 1(\mathrm{ppm})\end{array} & 90 & 80 & 70 & 60 & 50 & 40 & 30 & 20 & 10 & 0 & -10 & \end{array}$


AL-06-64.1.fid

1H AL-06-64

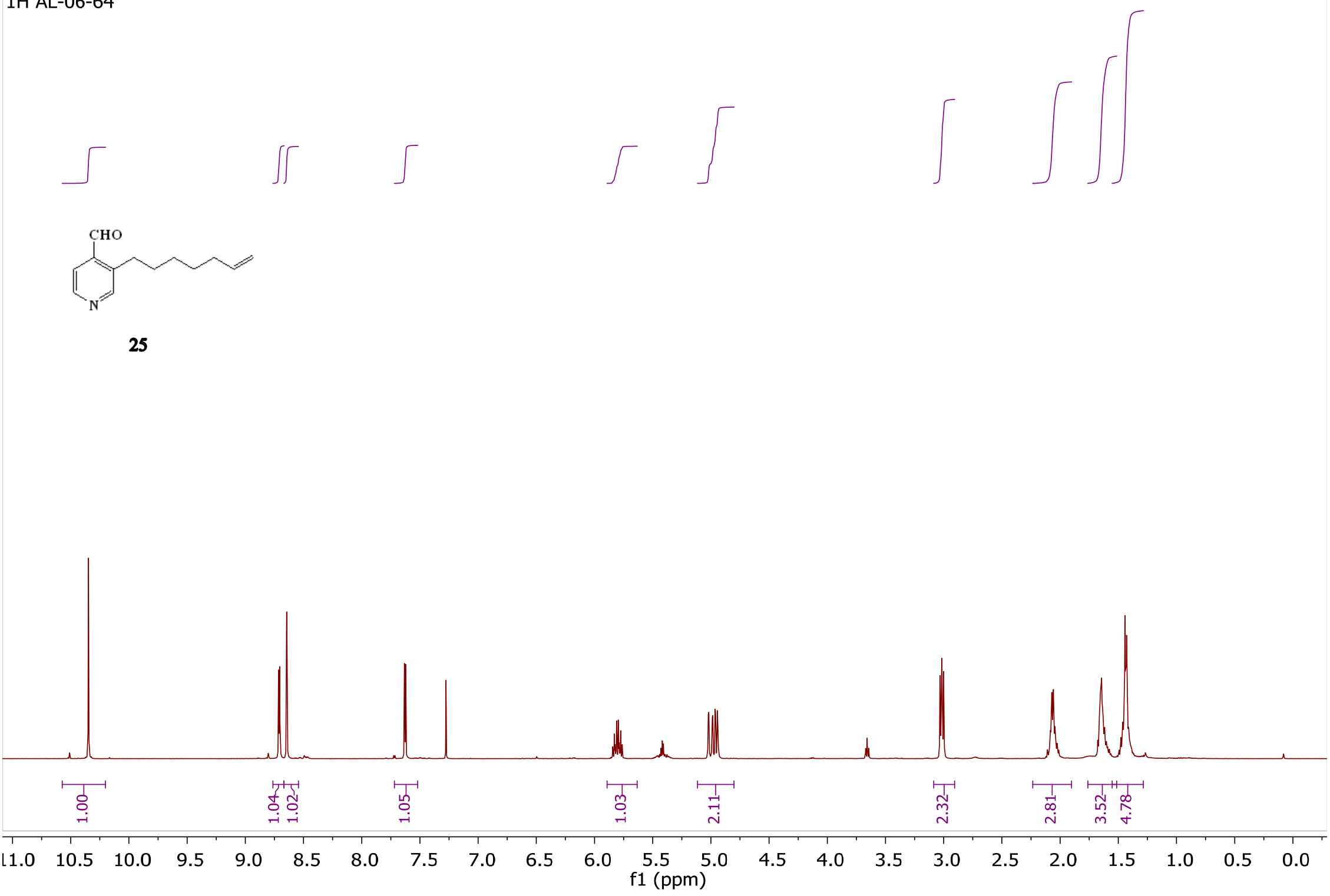




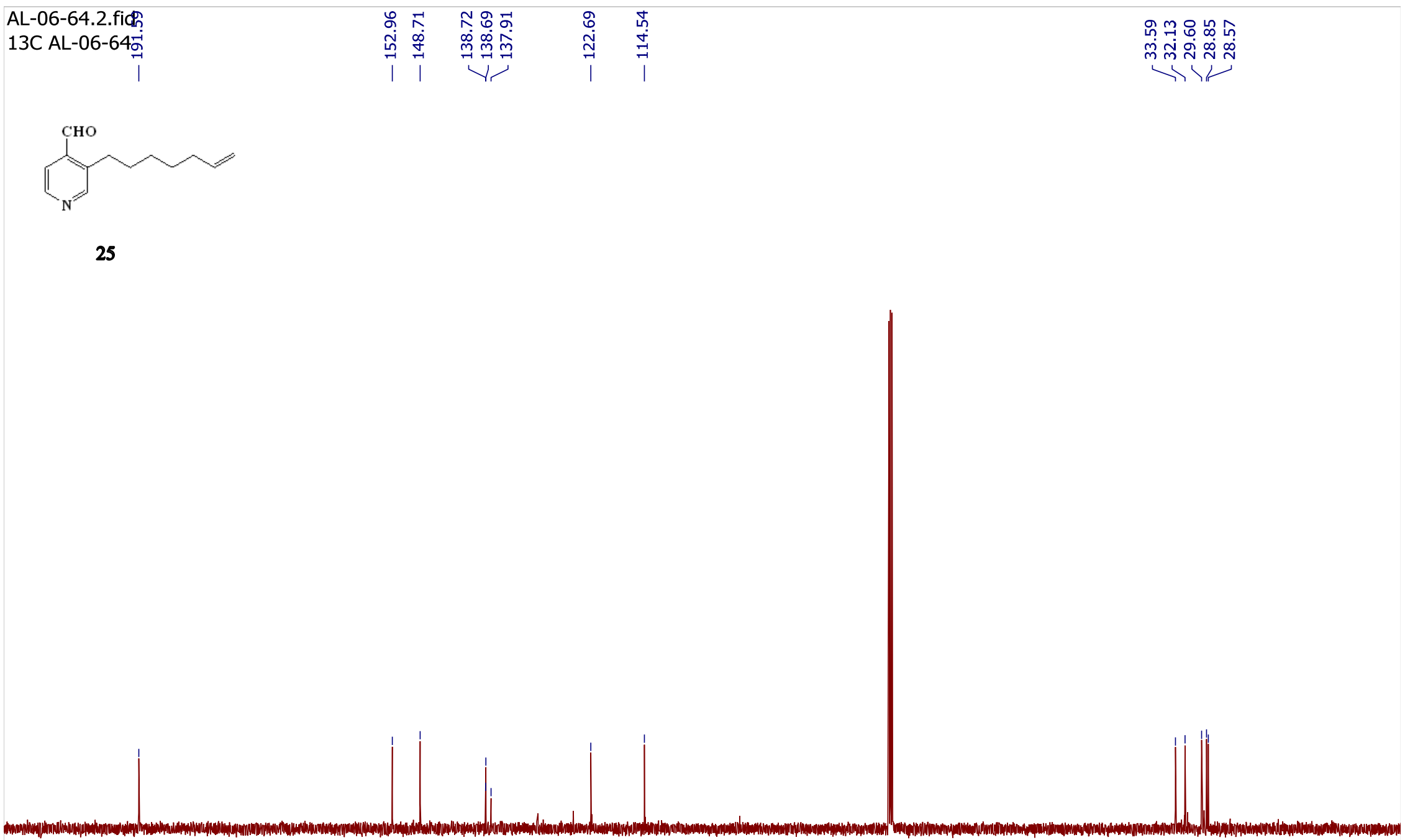

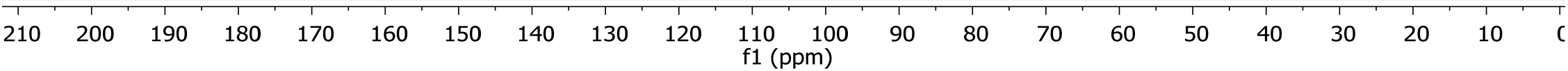


AL-05-238.1.fid

1H AL-05-238

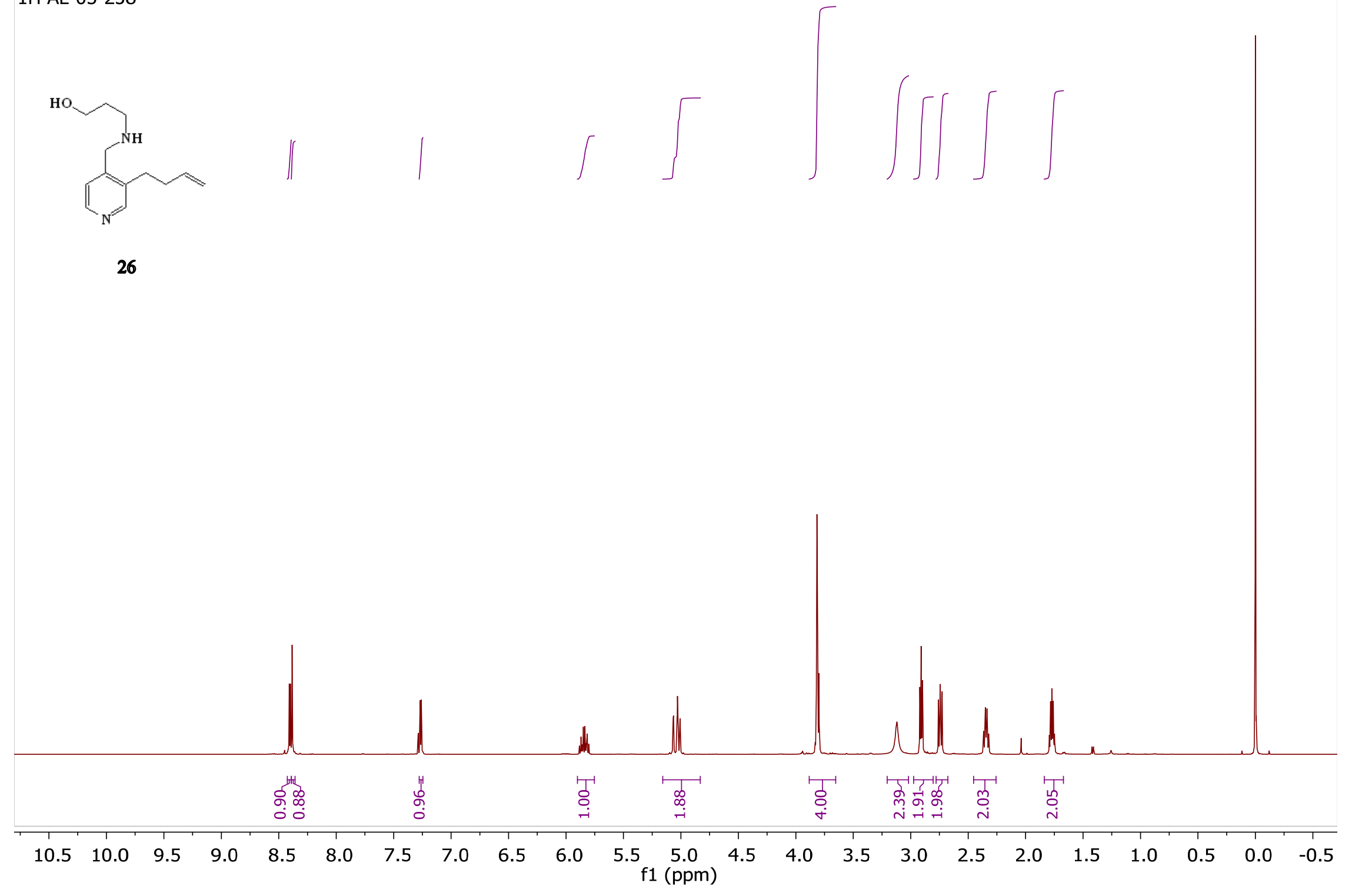



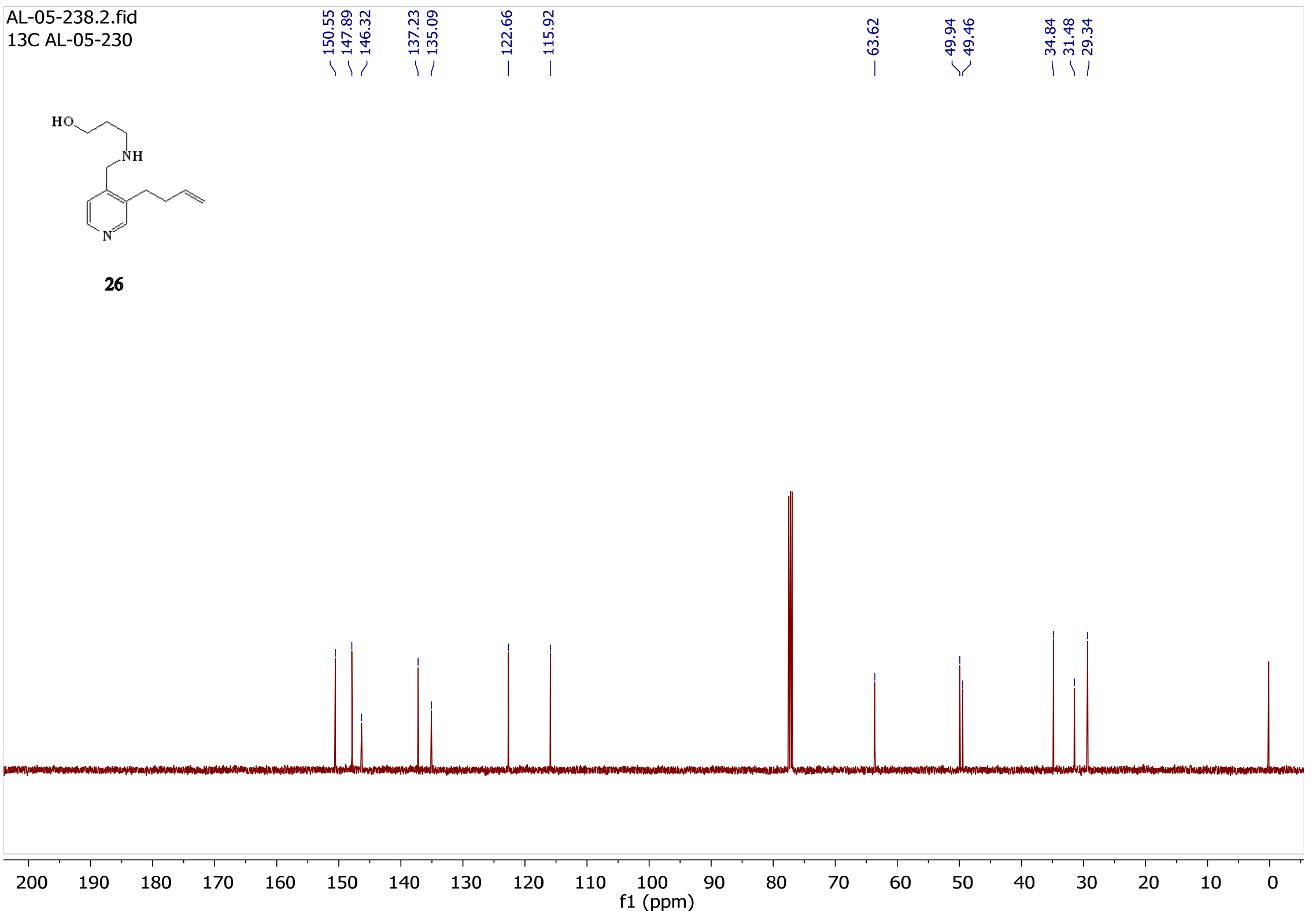
AL-04-292.1.fid

1H AL-04-292
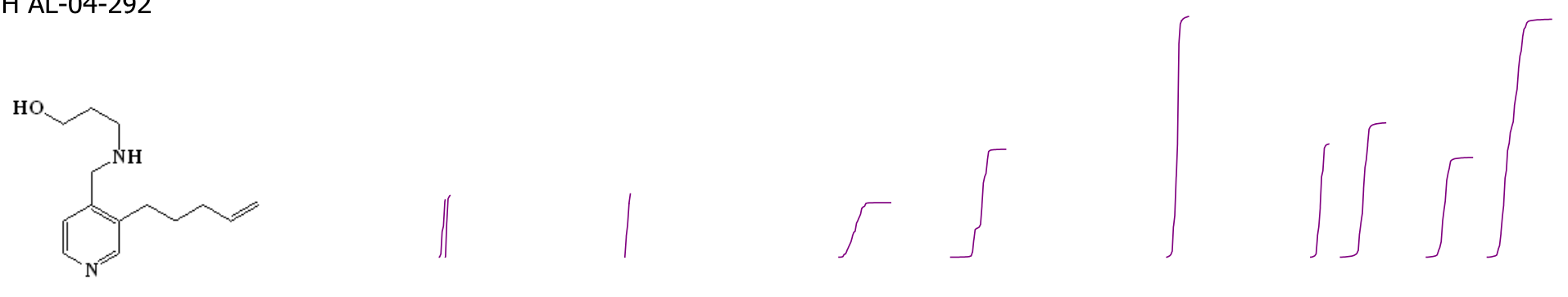

27

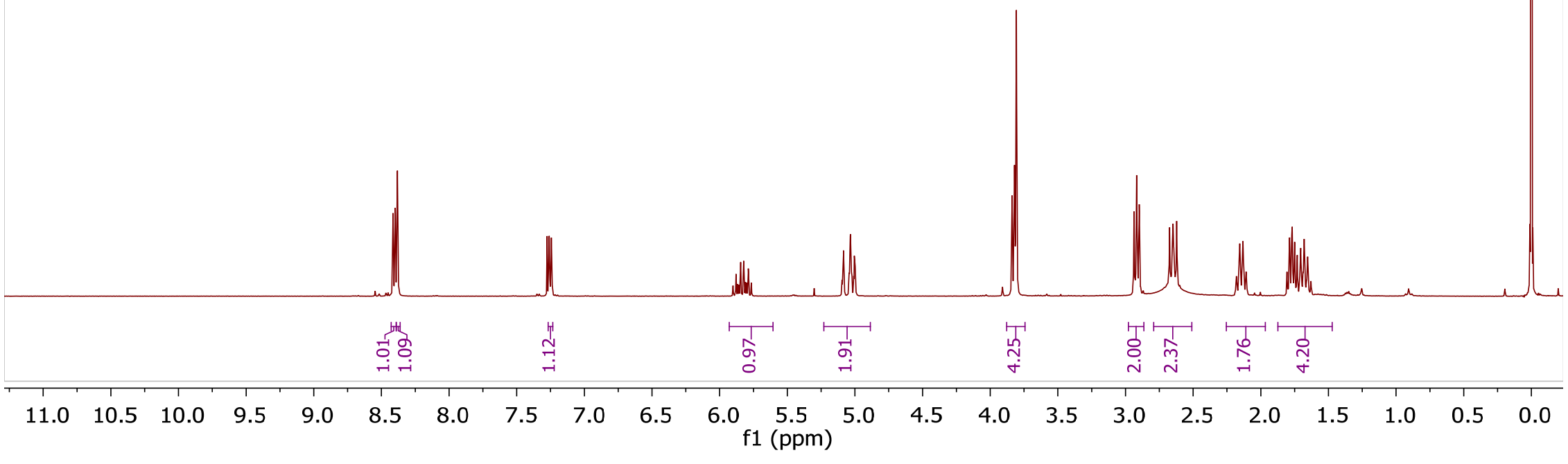




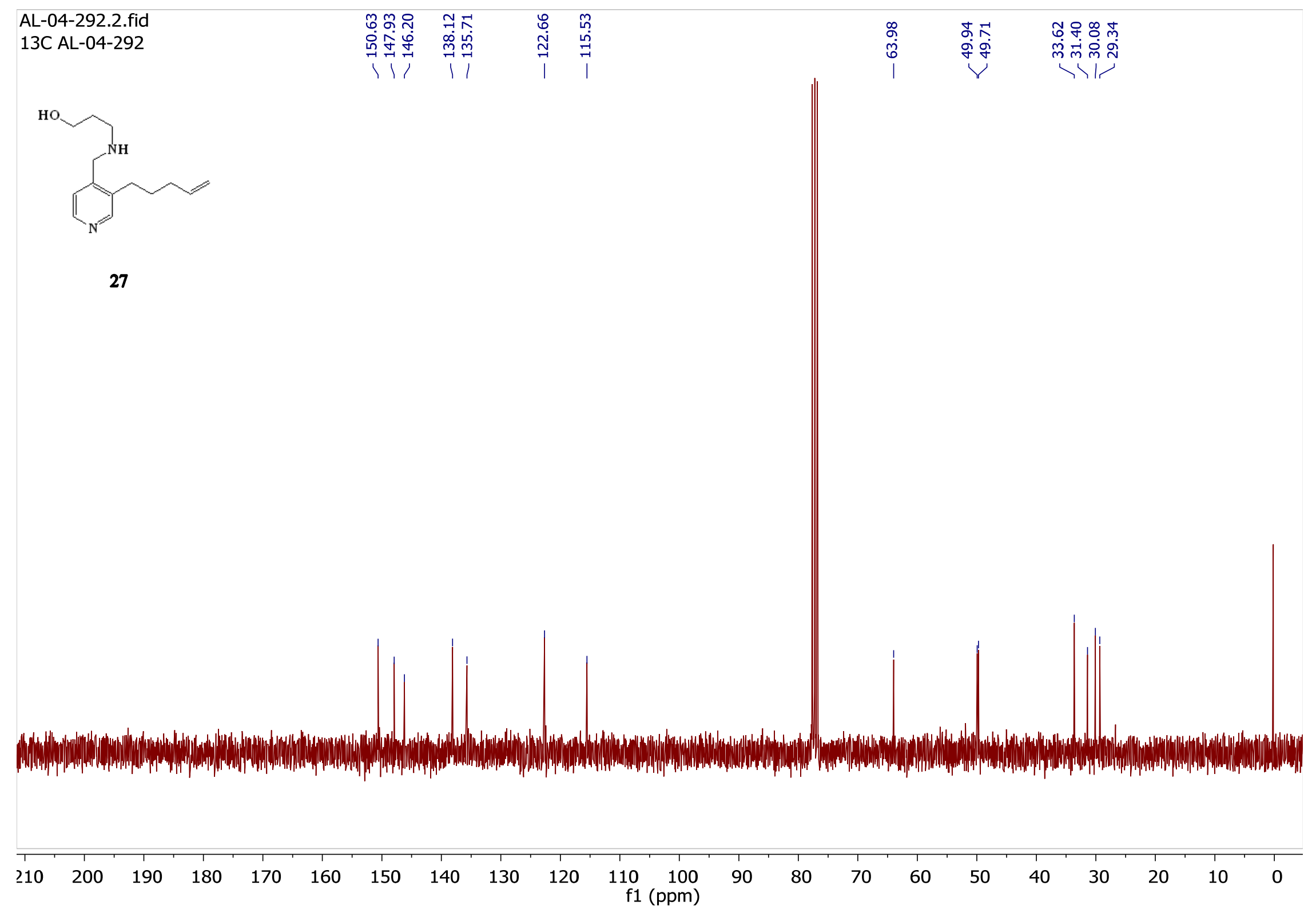


AL-05-212.1.fid

1H AL-05-212

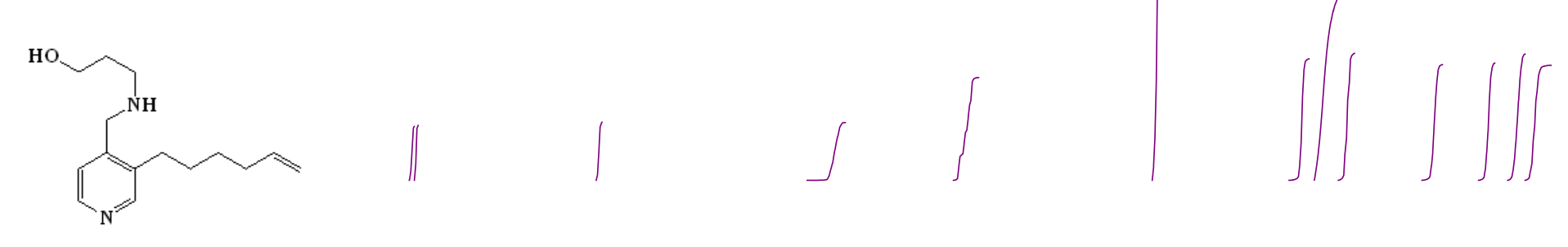

28

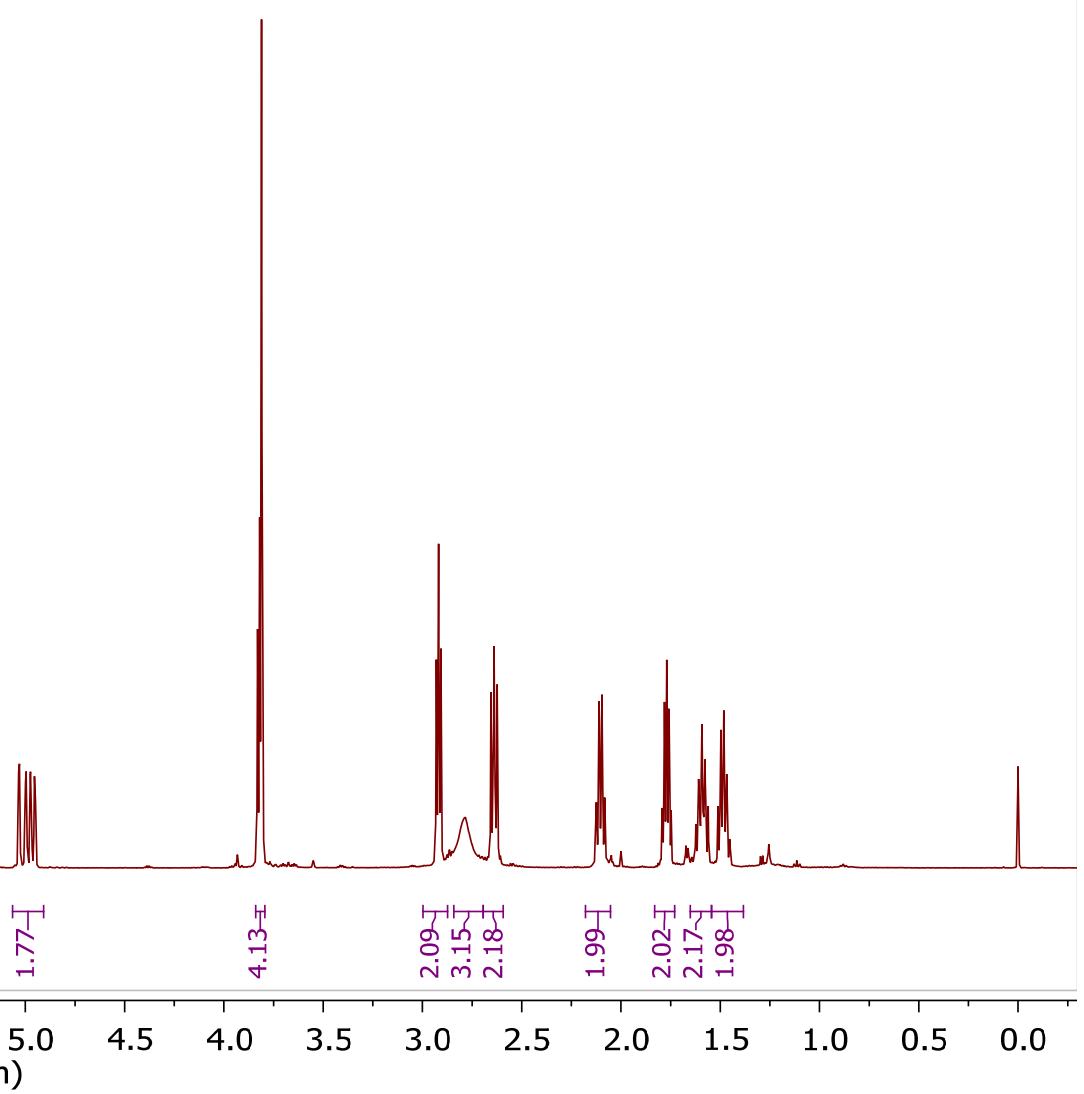




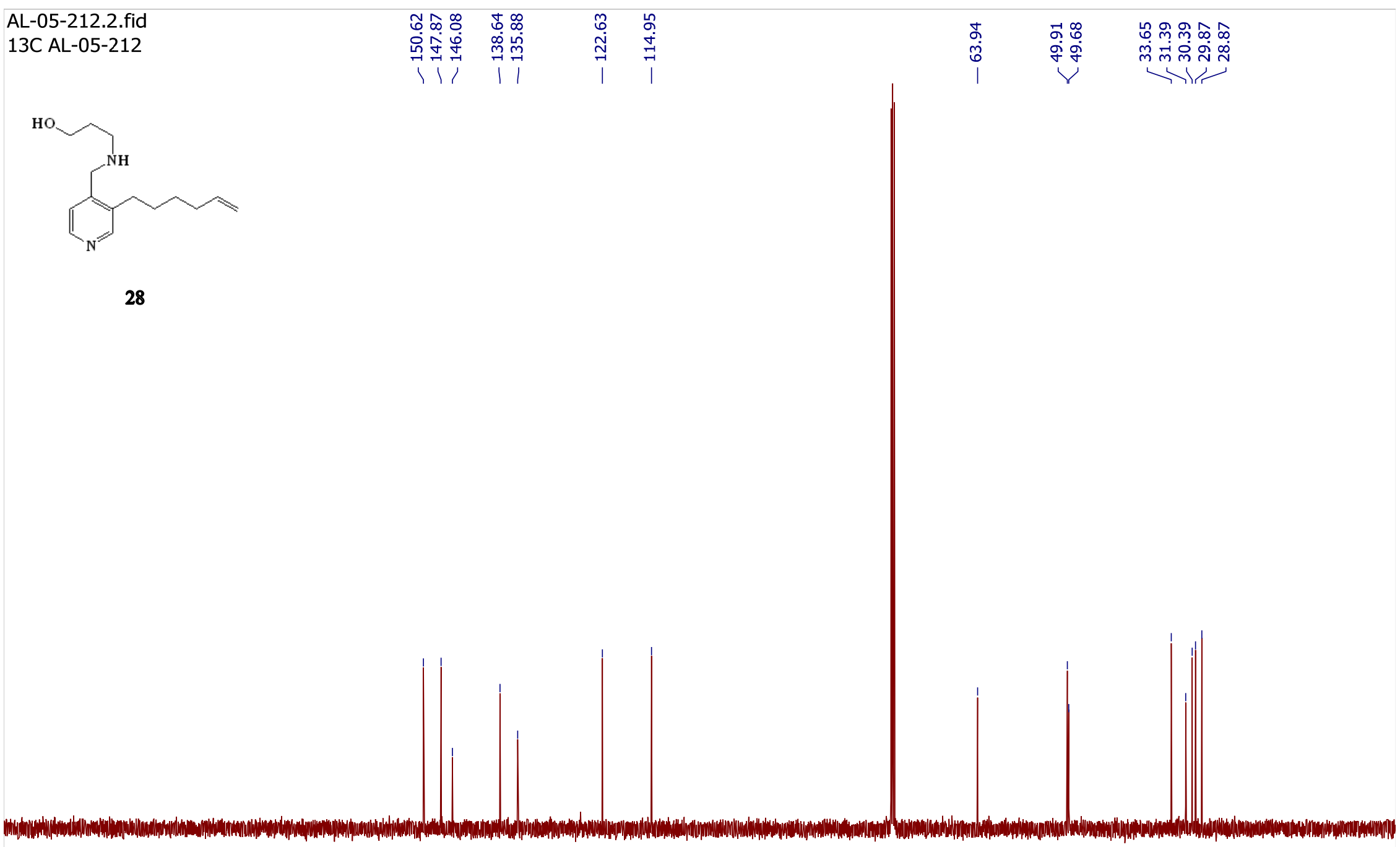

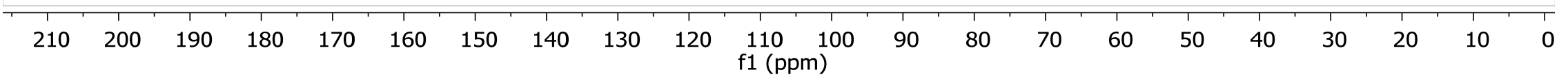


AL-11-84.1.fid

AL-11-84

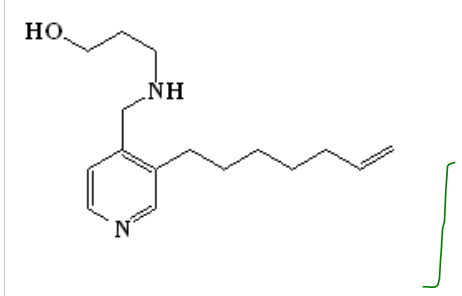

29
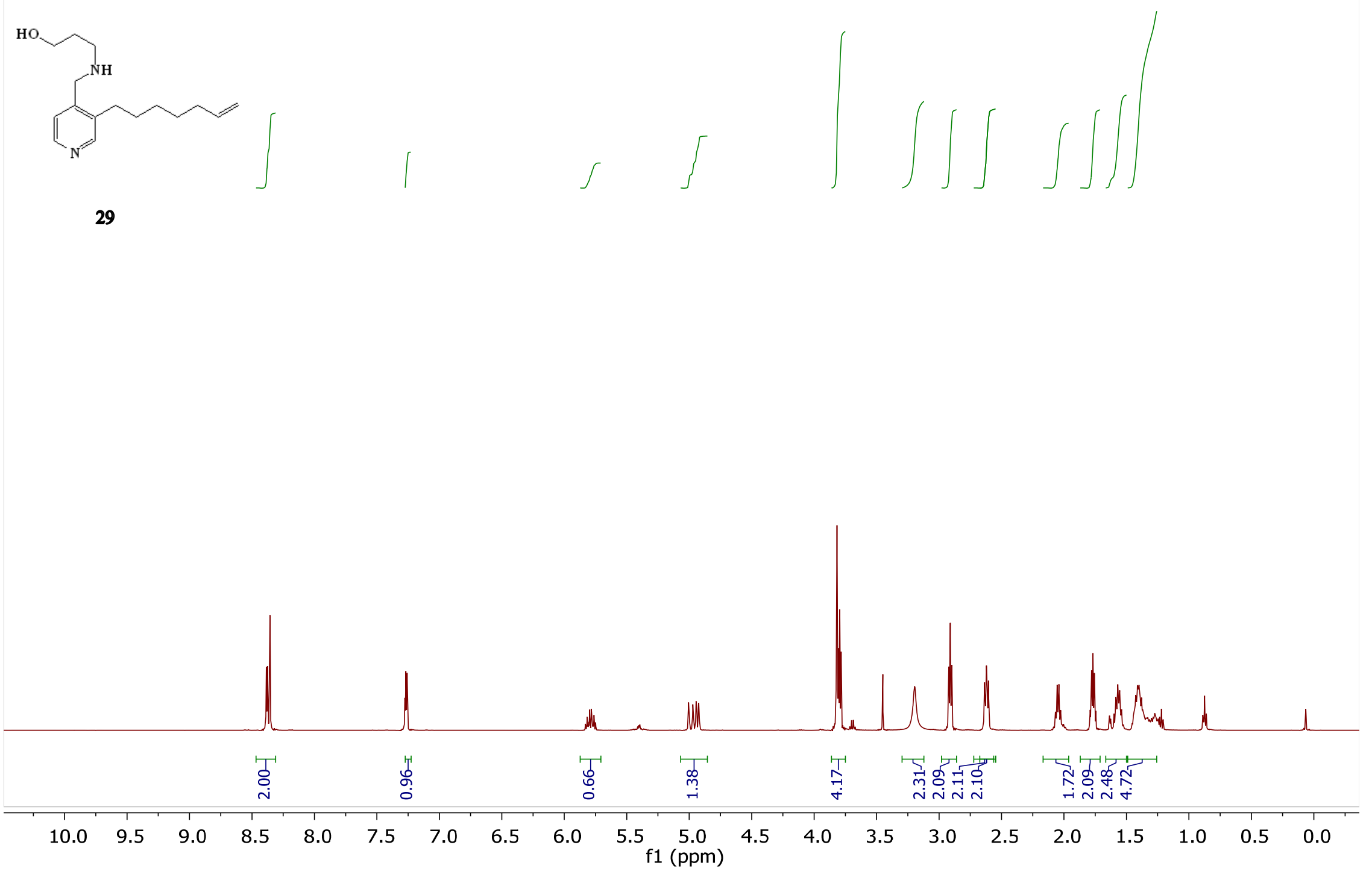

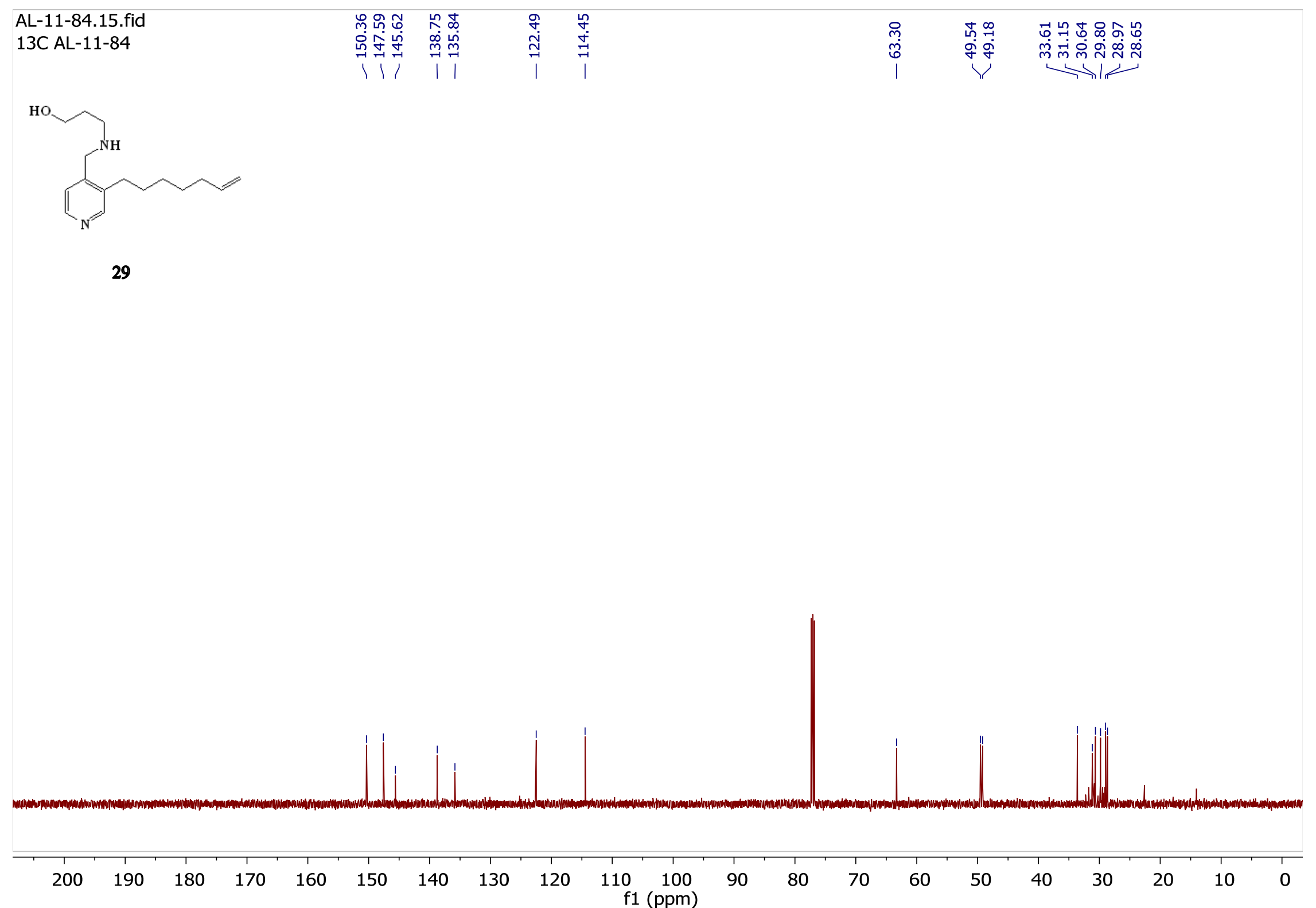


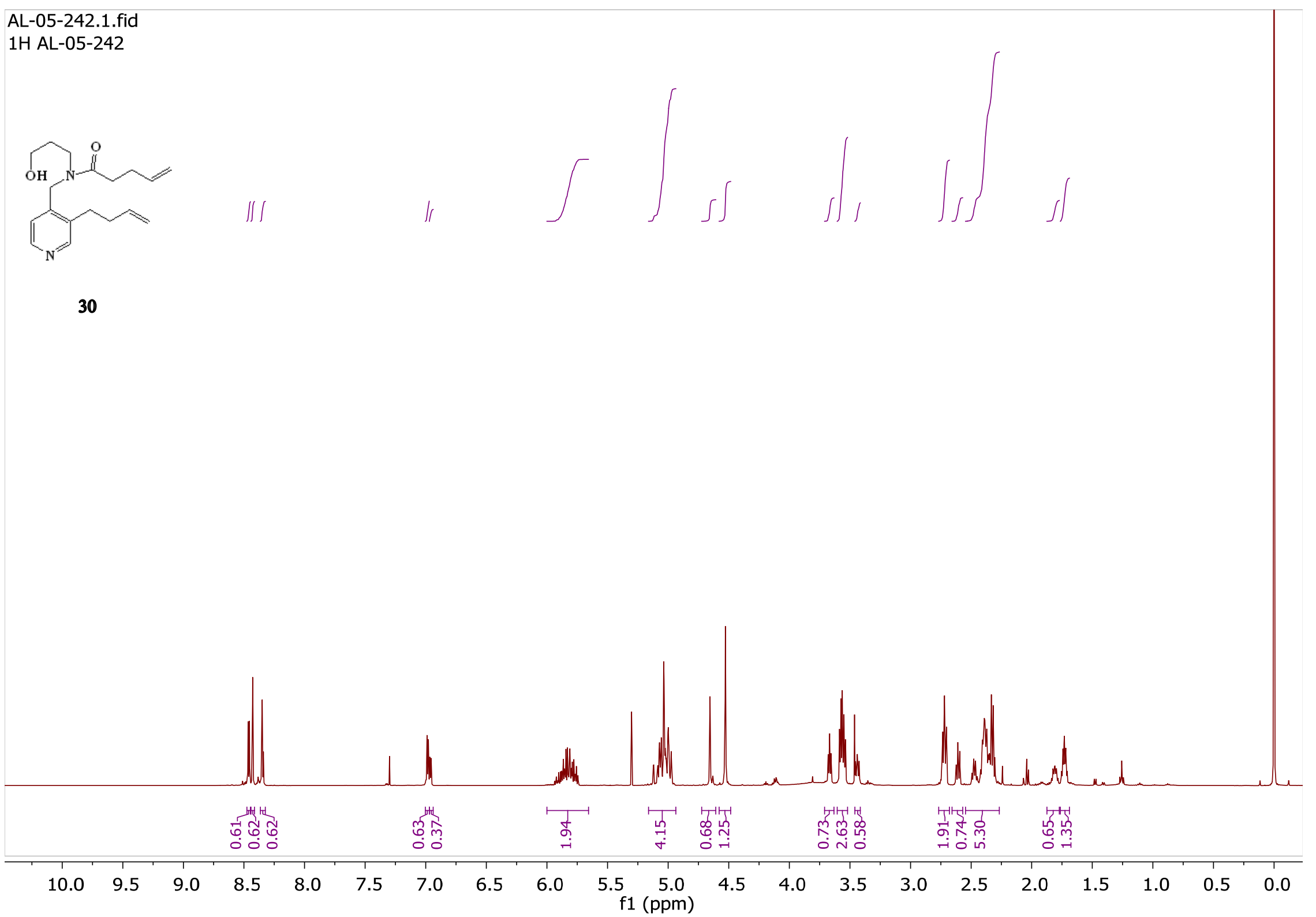


AL-05-242.2.fid 13C AL-05-242

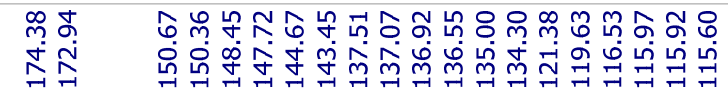

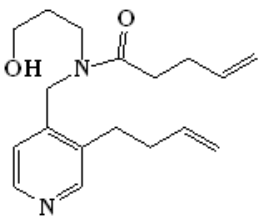

30

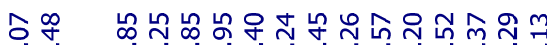

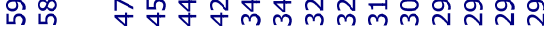

i) $\backslash, 4$

\begin{tabular}{|c|c|c|c|c|c|c|c|c|c|c|c|c|c|c|c|c|c|c|c|c|}
\hline 10 & 200 & 190 & 180 & 170 & 160 & 150 & 140 & 130 & 120 & 110 & $\begin{array}{r}100 \\
(\mathrm{ppm})\end{array}$ & 90 & 80 & 70 & 60 & 50 & 40 & 30 & 20 & 10 \\
\hline
\end{tabular}




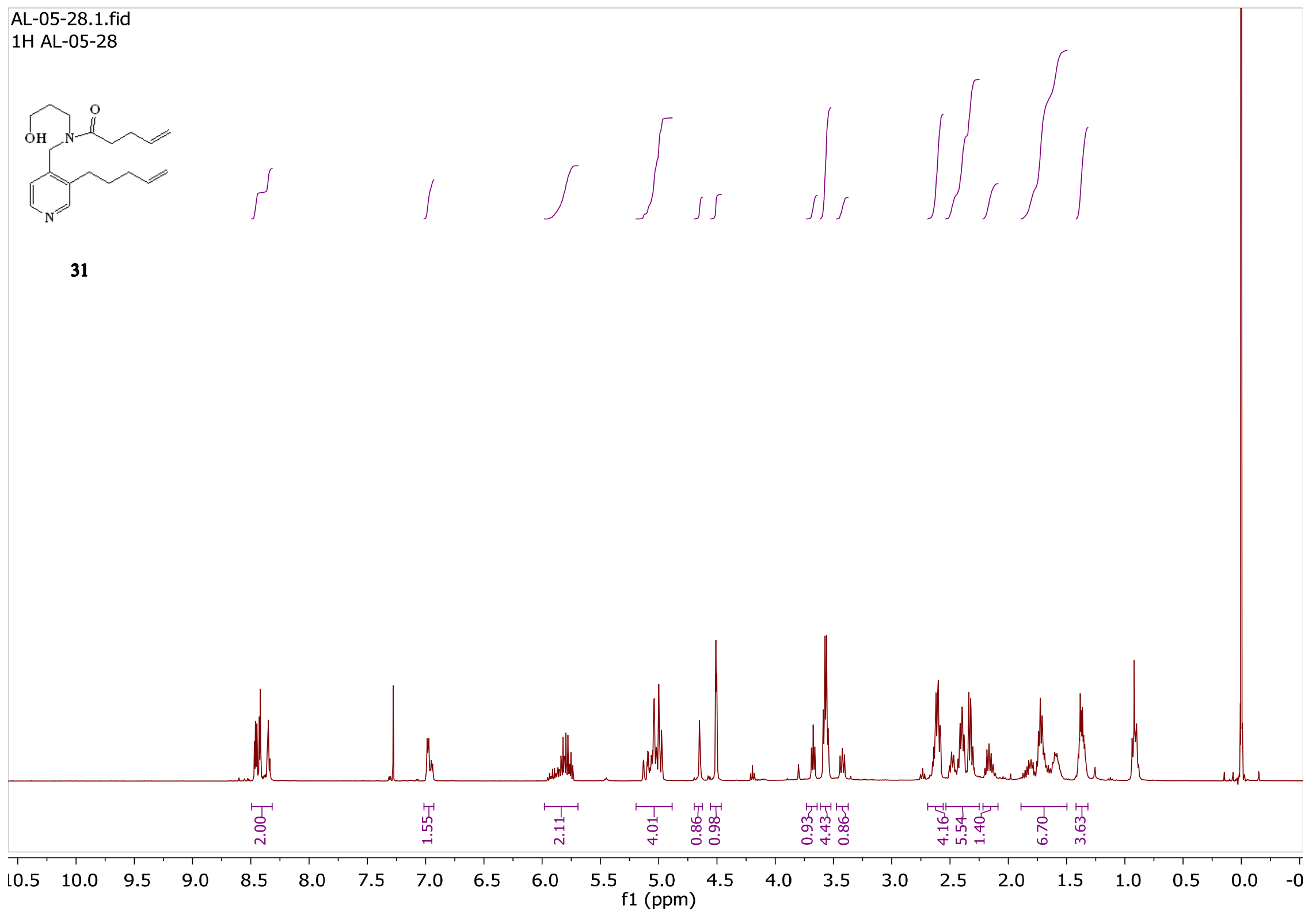

S41 


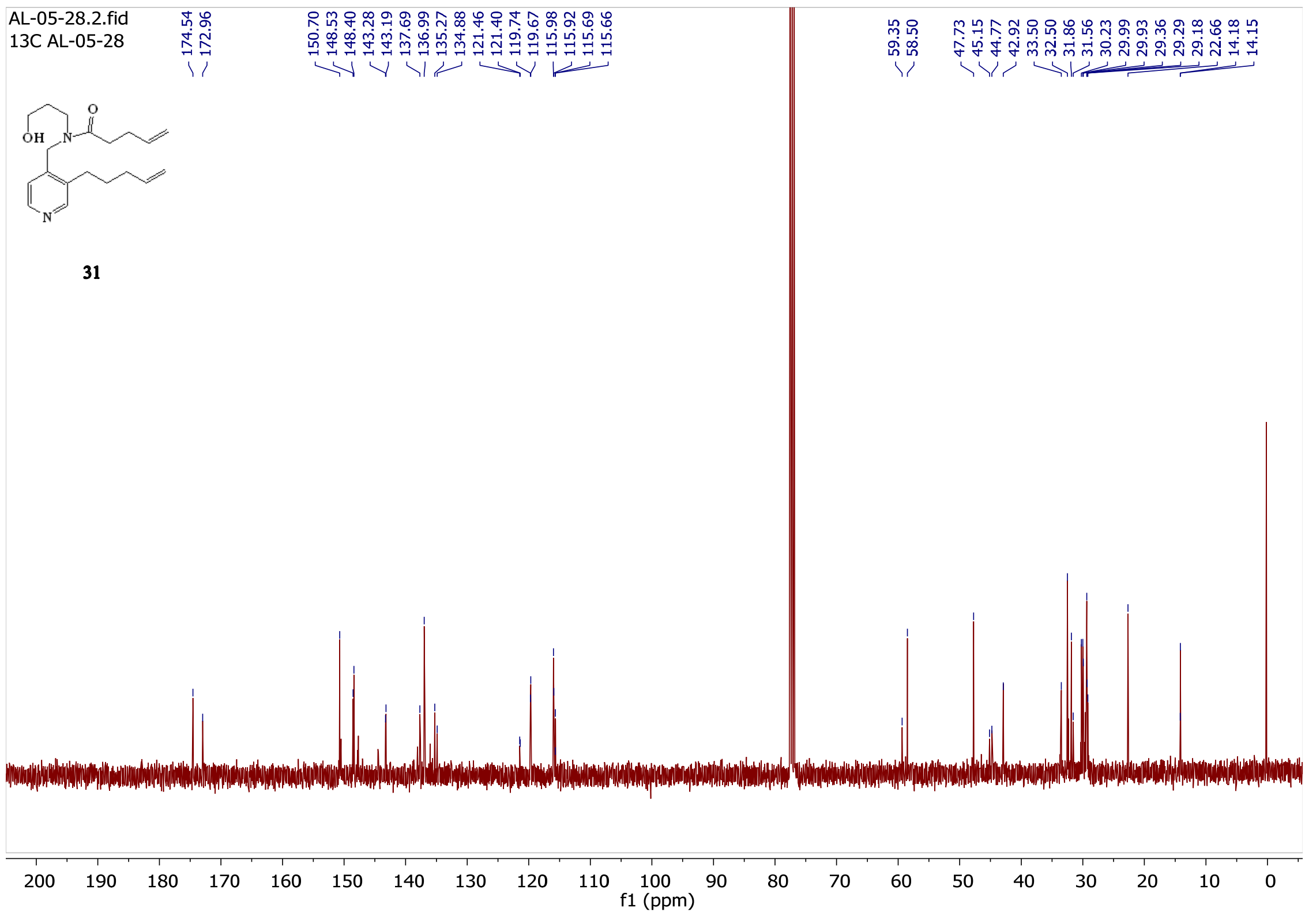




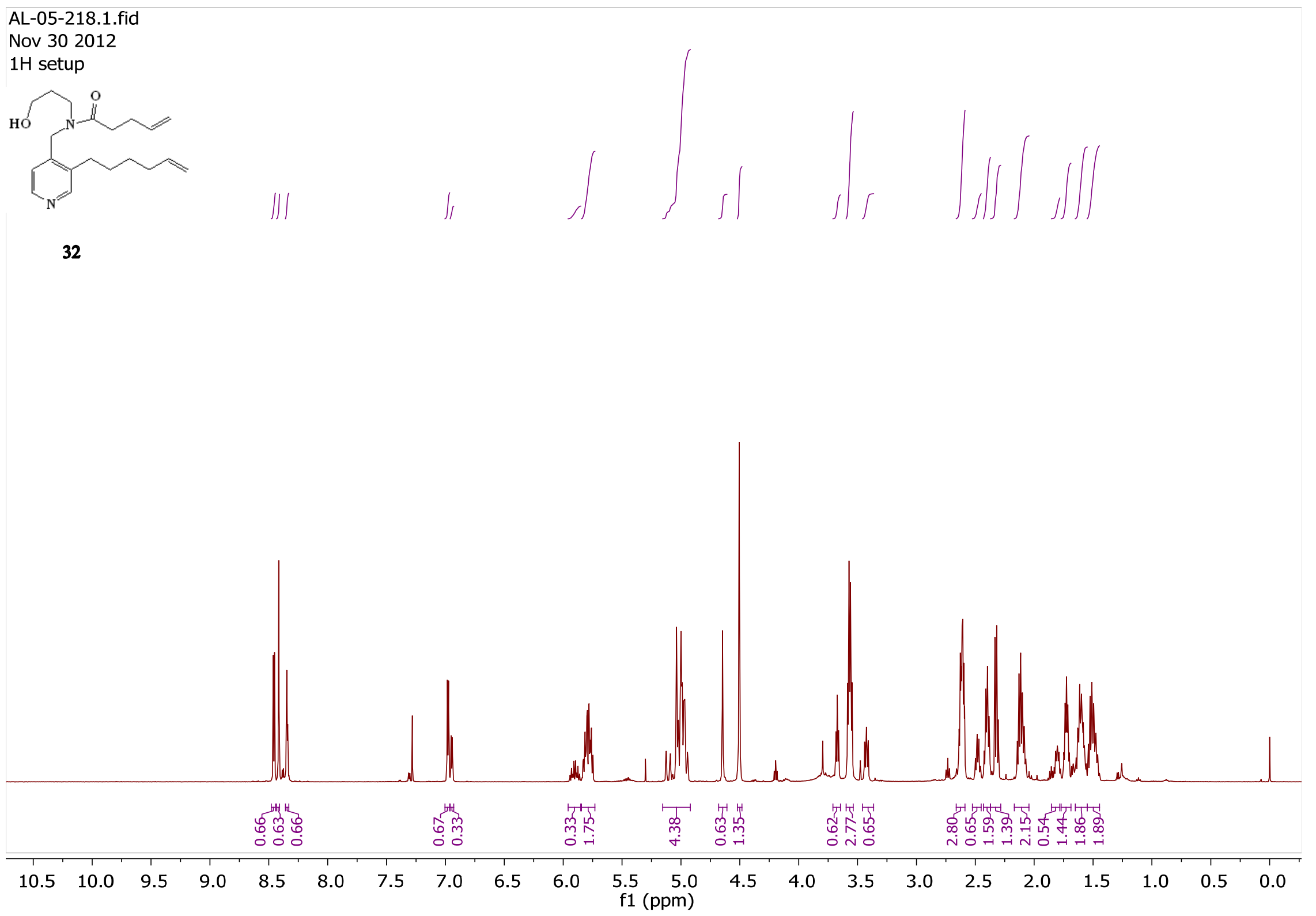


AL-05-218.2.fid 13C AL-05-218

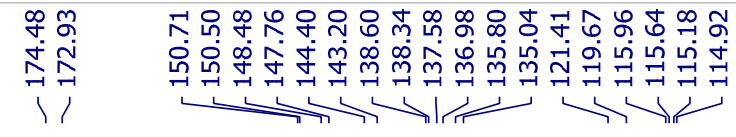

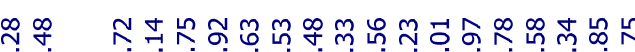

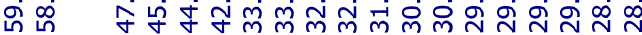

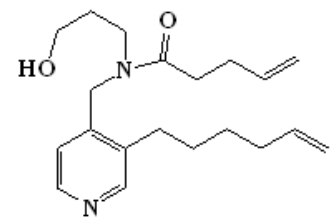

32 


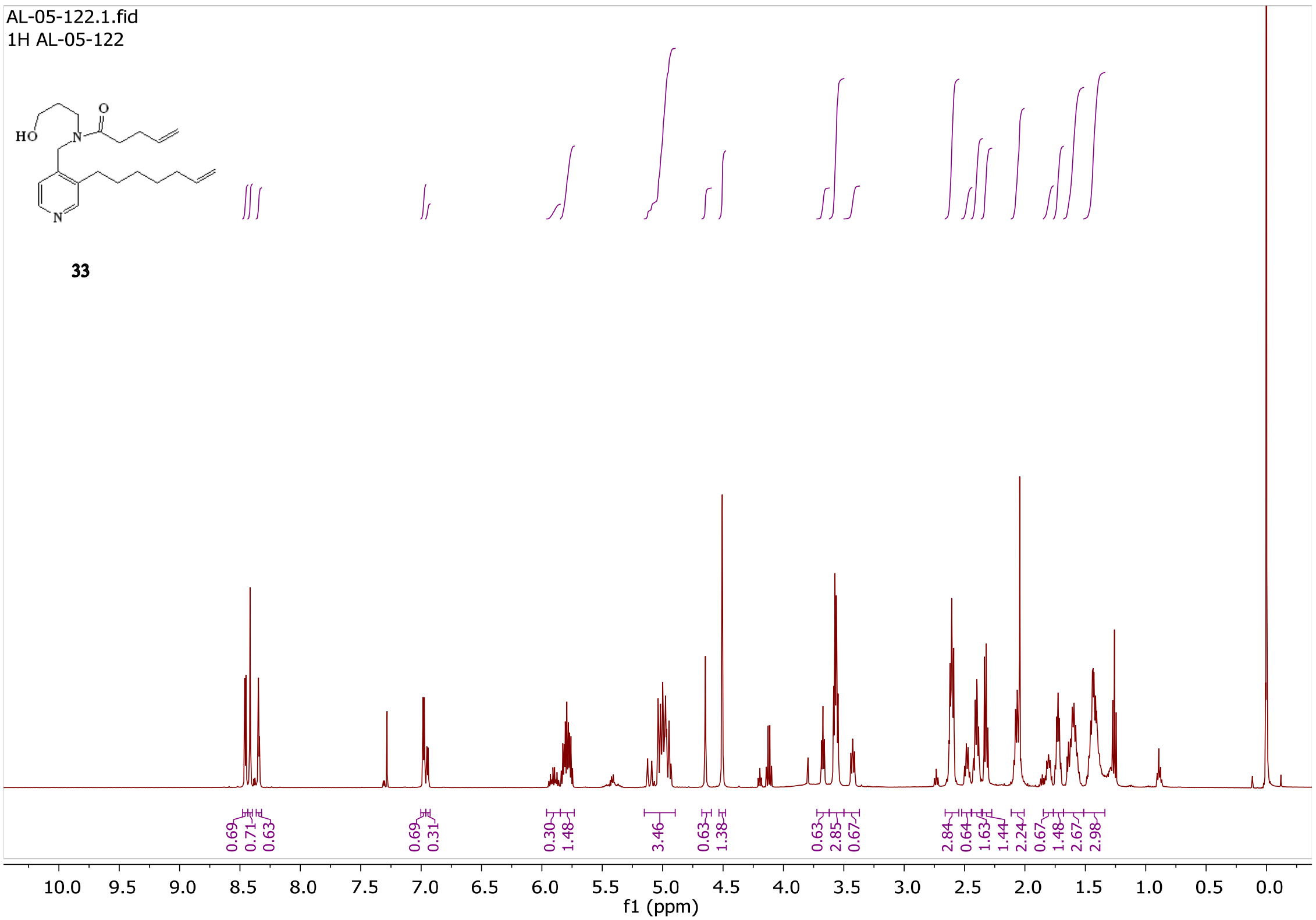




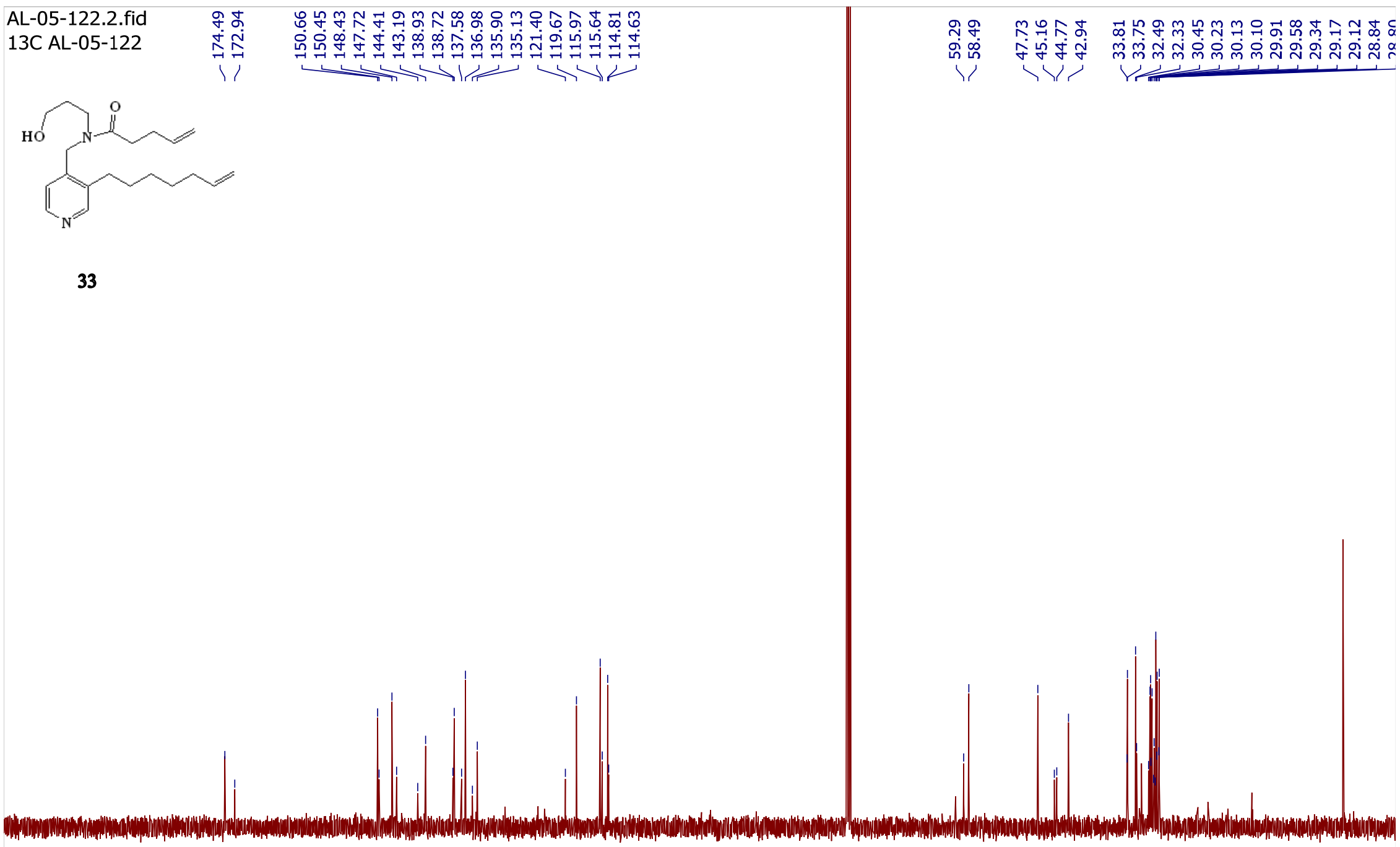

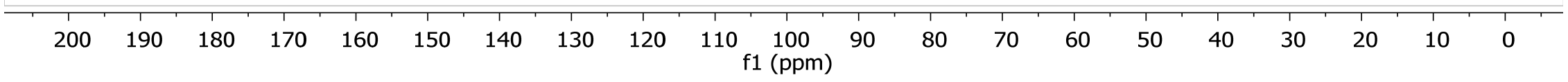


AL-06-60 Spot 2.1.fid 1H AL-06-60 Spot 2
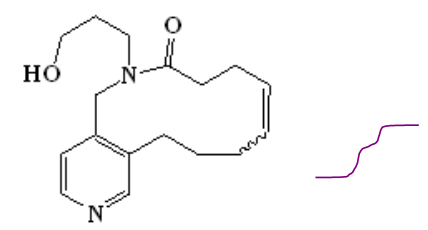

36

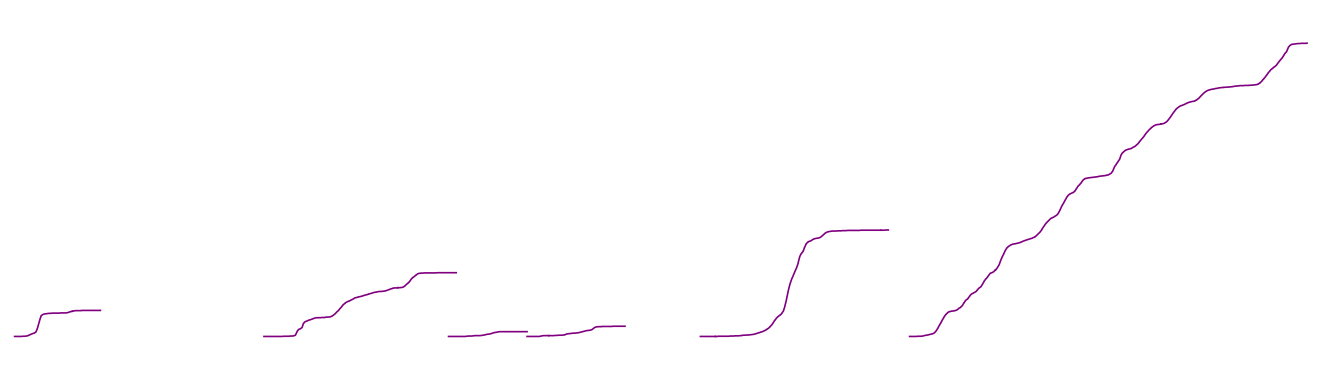

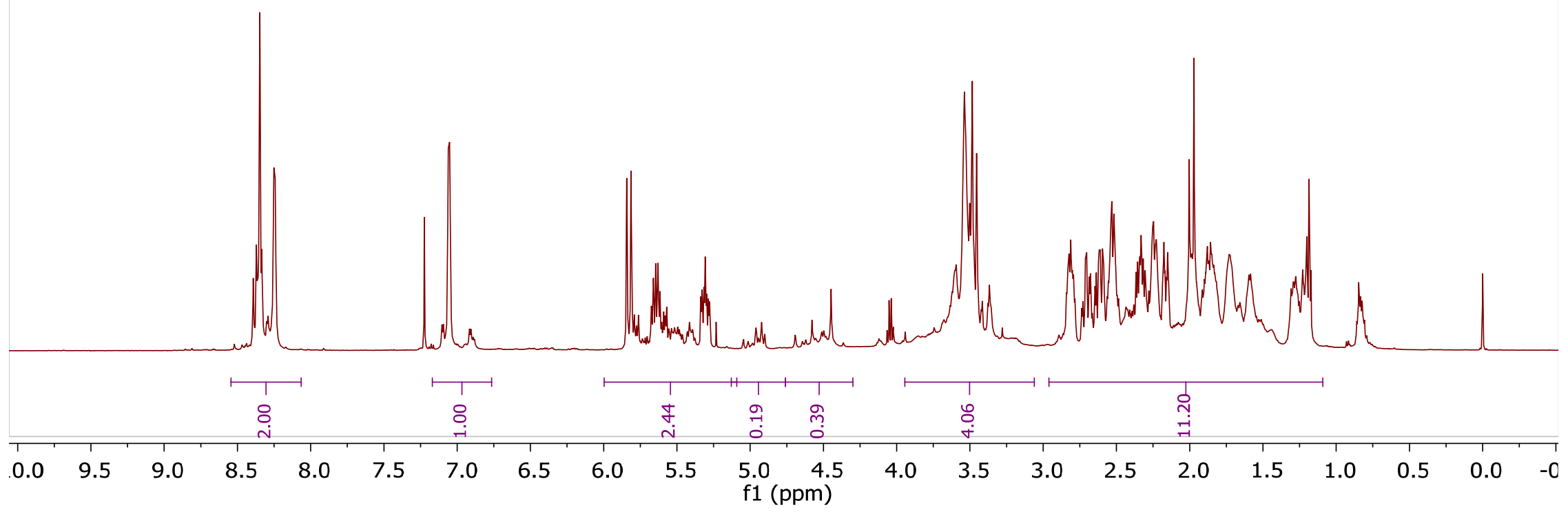

S47 
AL-06-60 Spot 2.2.fid 13C AL-06-60 Spot 2

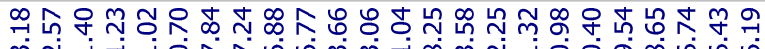

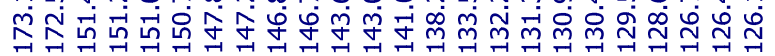

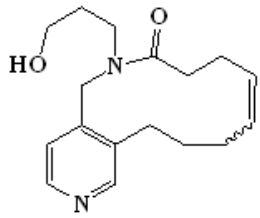

36

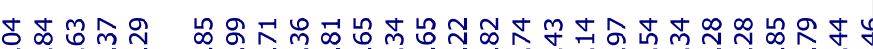

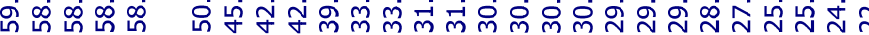

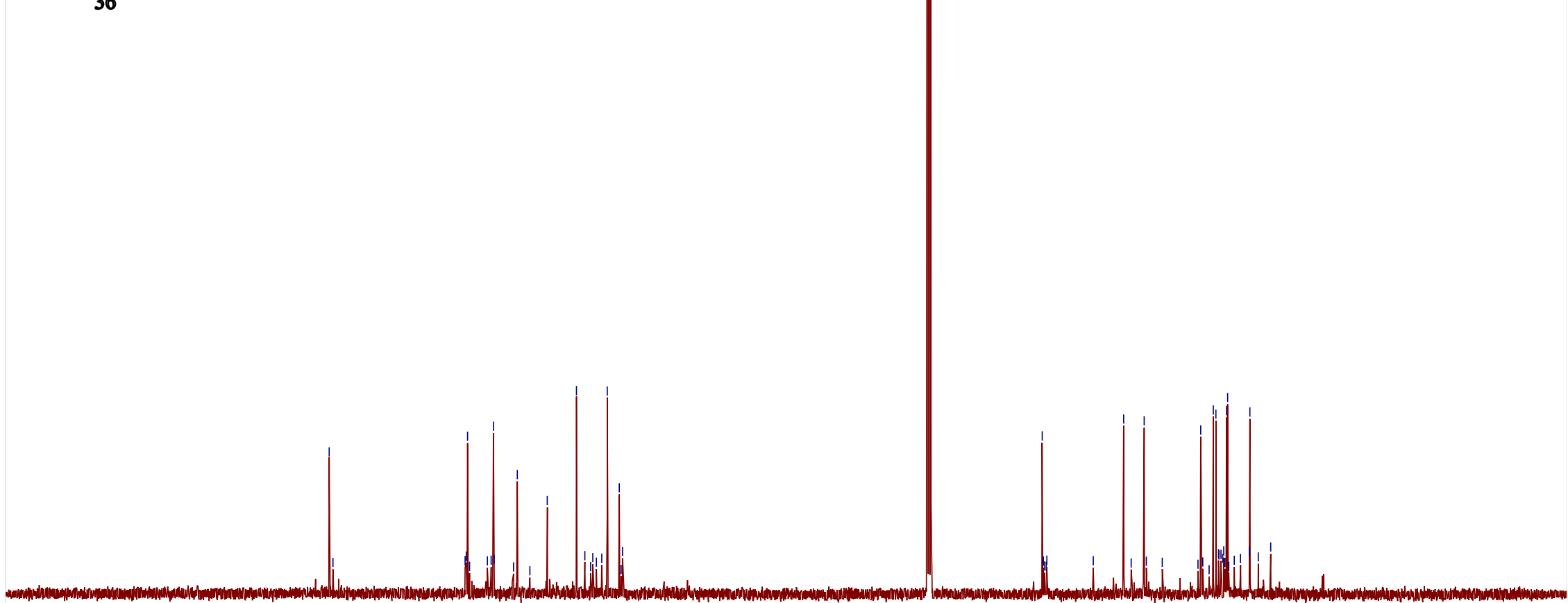

$\begin{array}{lllllllllllllllllllllllll}220 & 210 & 200 & 190 & 180 & 170 & 160 & 150 & 140 & 130 & 120 & 110 & \begin{array}{l}100 \\ \mathrm{f} 1(\mathrm{ppm})\end{array} & 90 & 80 & 70 & 60 & 50 & 40 & 30 & 20 & 10 & 0 & -10 & -20\end{array}$


AL-05-230.1.fid

1H AL-05-230

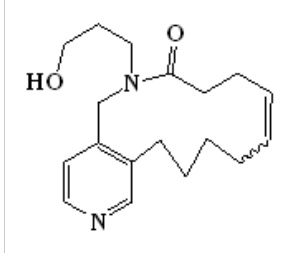

37
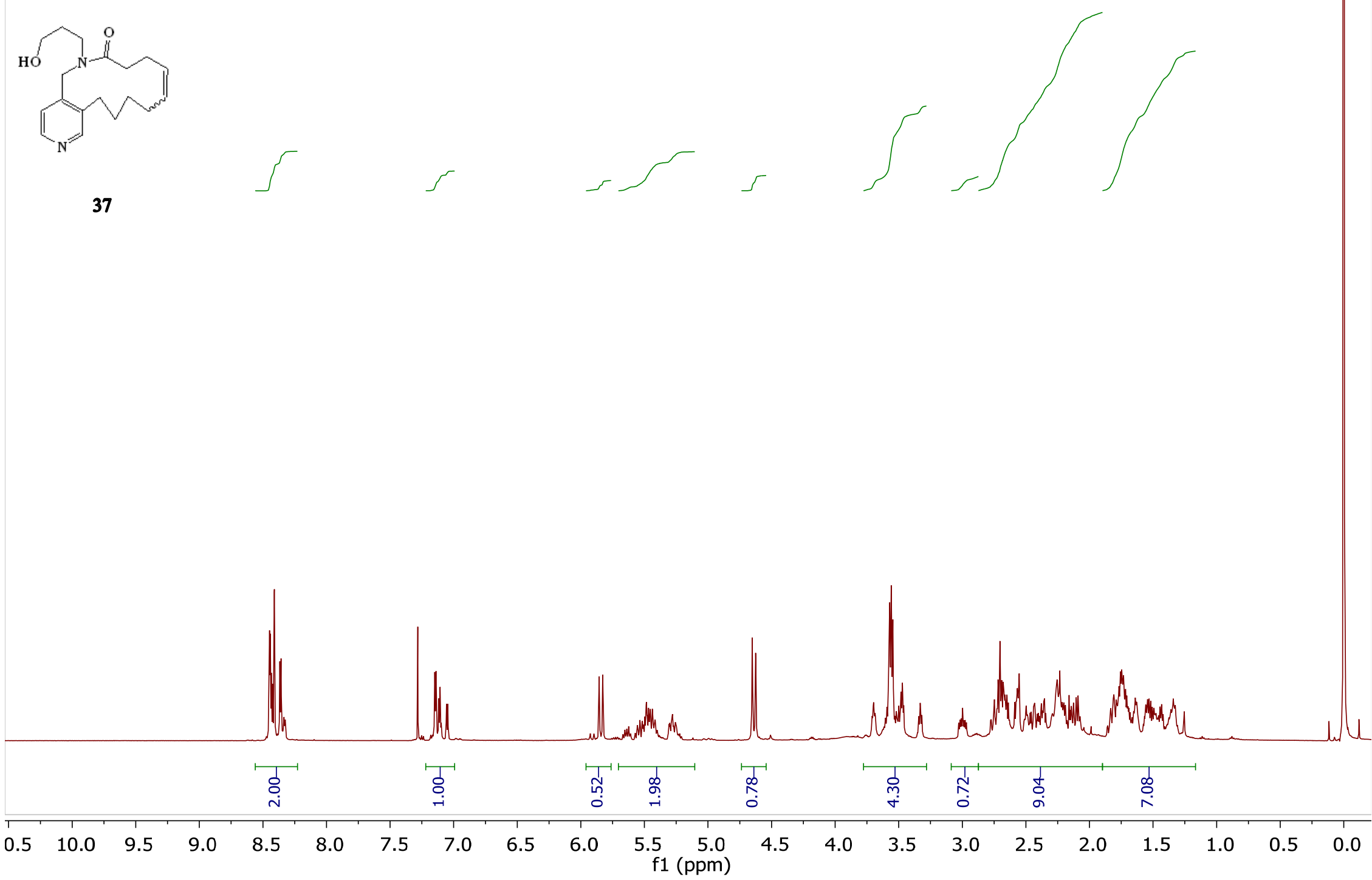
AL-05-230/2

13C AL-05-230

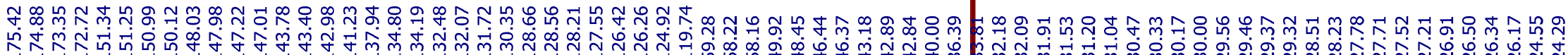
न<smiles>O=C(CC/C=C\CCCCc1cnccc1CN(CCO)CCCO)N1CCCCC1</smiles>

37

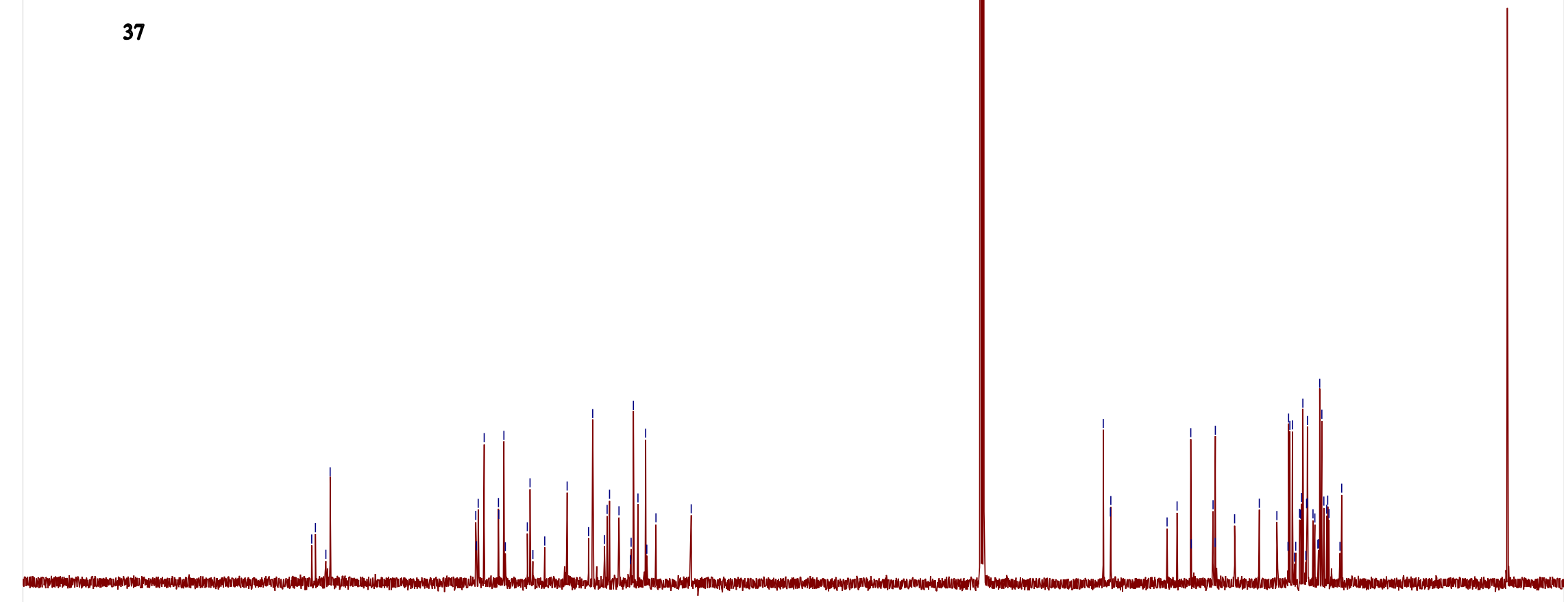

190180

$170 \quad 160$

150

$140 \quad 130$

120

$\begin{array}{cr}110 & 100 \\ \mathrm{f} 1(\mathrm{ppm})\end{array}$

90

80

7060

$50 \quad 40$
0

3020

0 


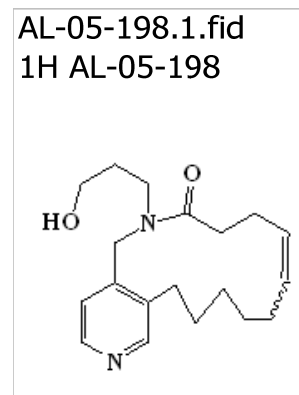

34

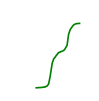

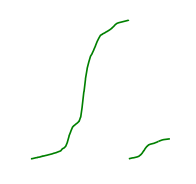

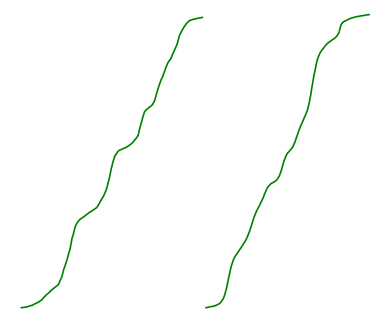

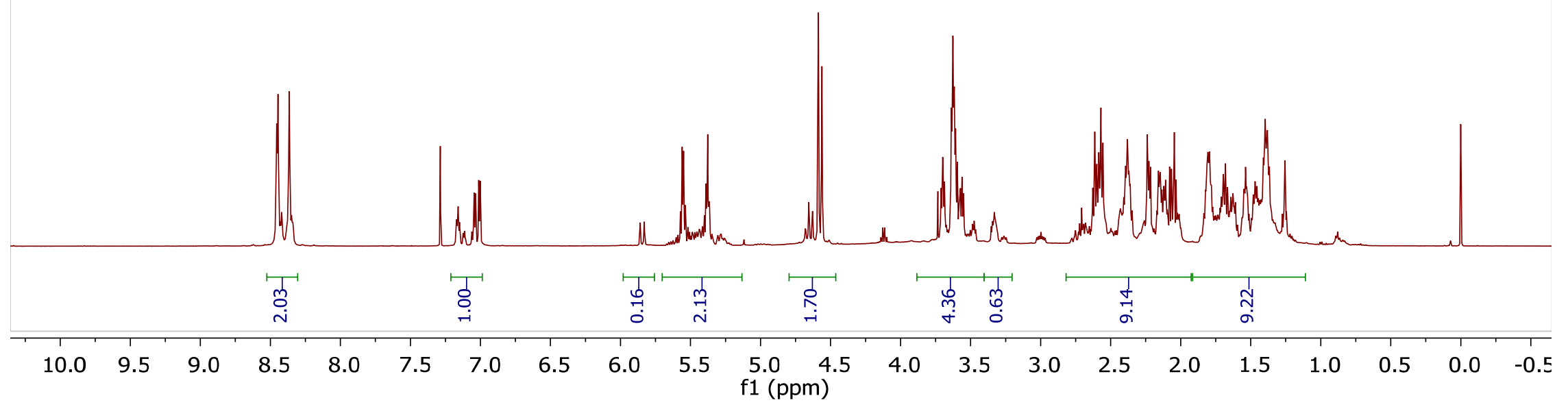

S51 
AL-05-198.2.fid

13C AL-05-198

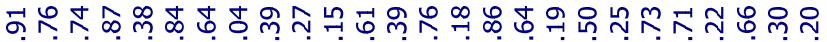

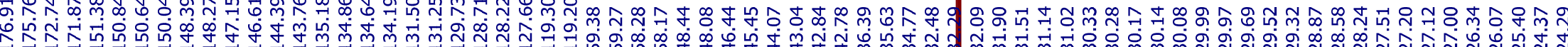

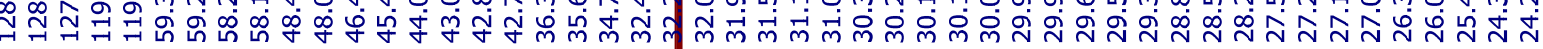

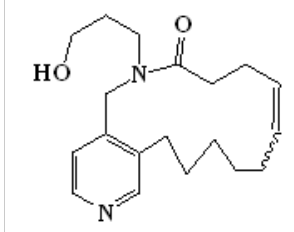

34

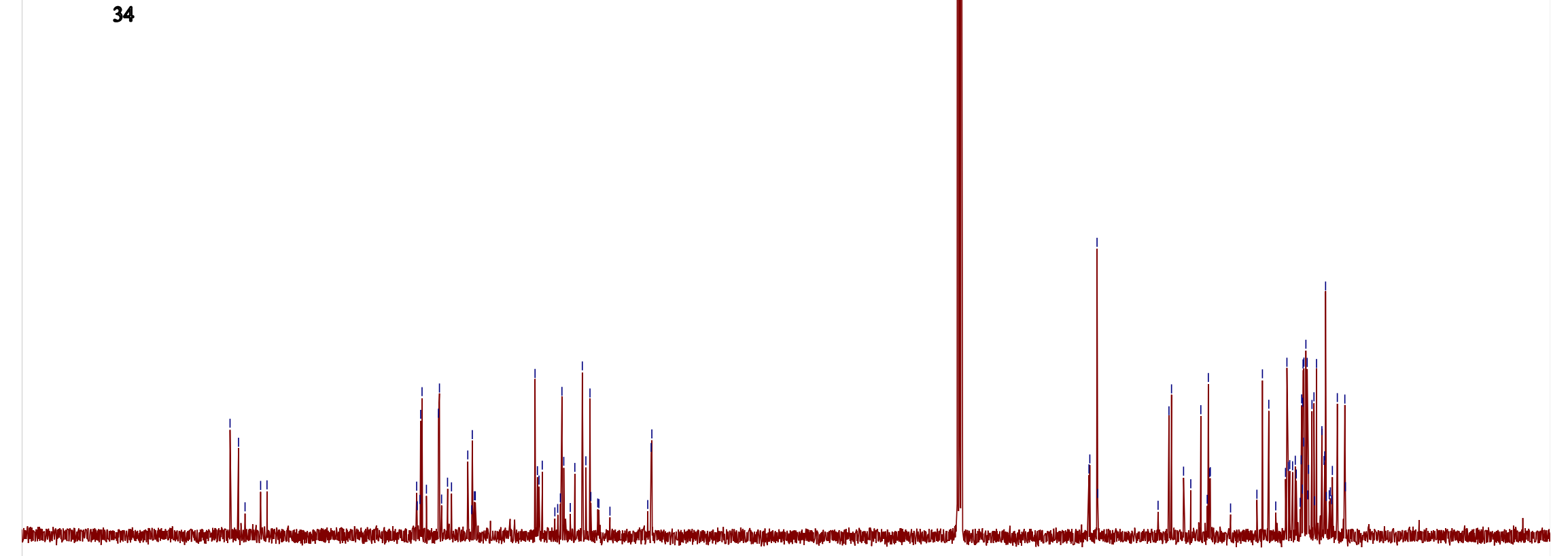

200

190

180

70160

150

140

130

120

$110 \begin{gathered}100 \\ \mathrm{f} 1(\mathrm{ppm})\end{gathered}$

80

70

60

50

$40 \quad 30$

20

10 
AL-06-66.1.fid

1H AL-06-66

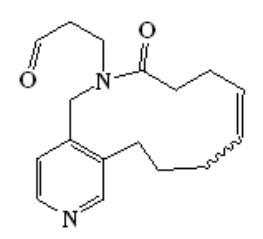

38

$38-$

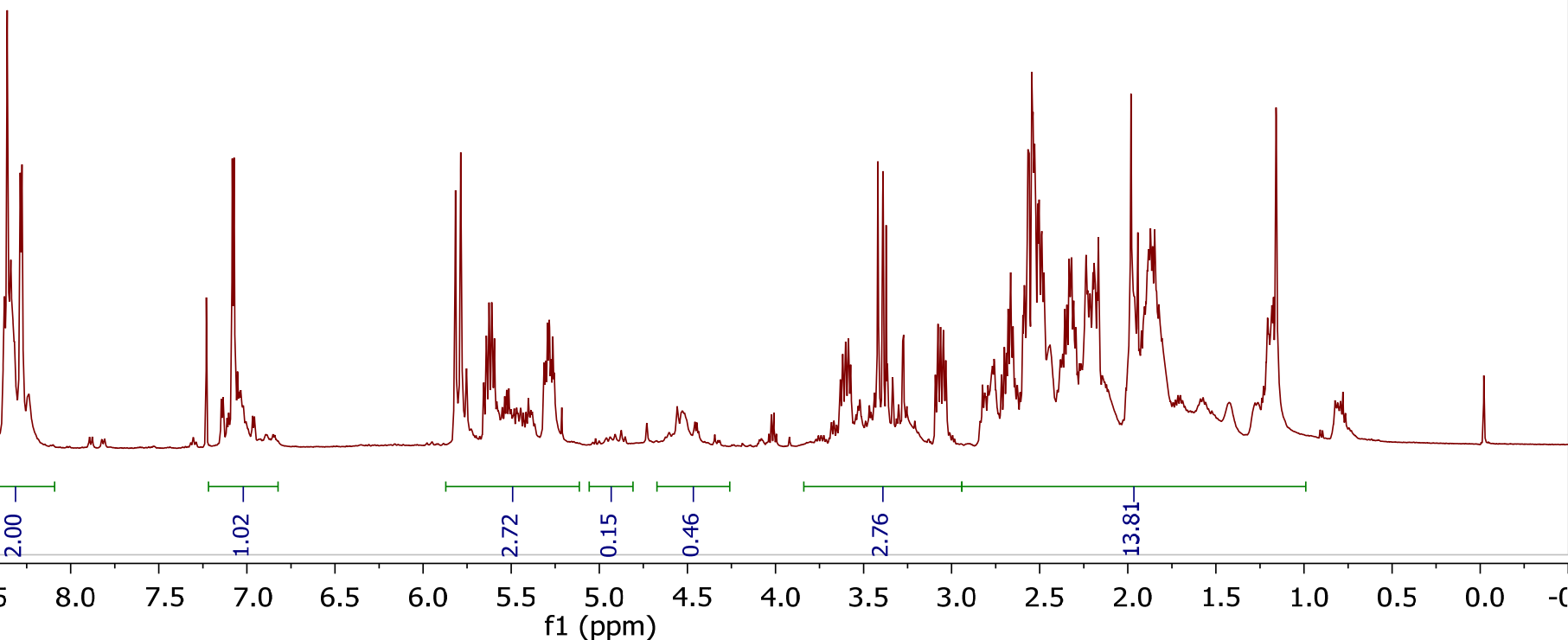


AL-06-66.2.fid

13C AL-06-66

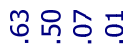

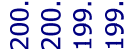

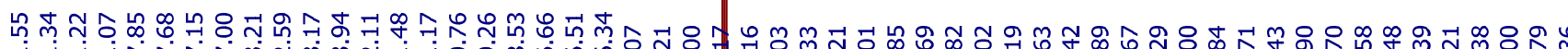

4

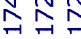

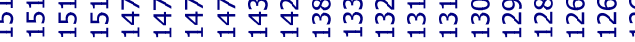
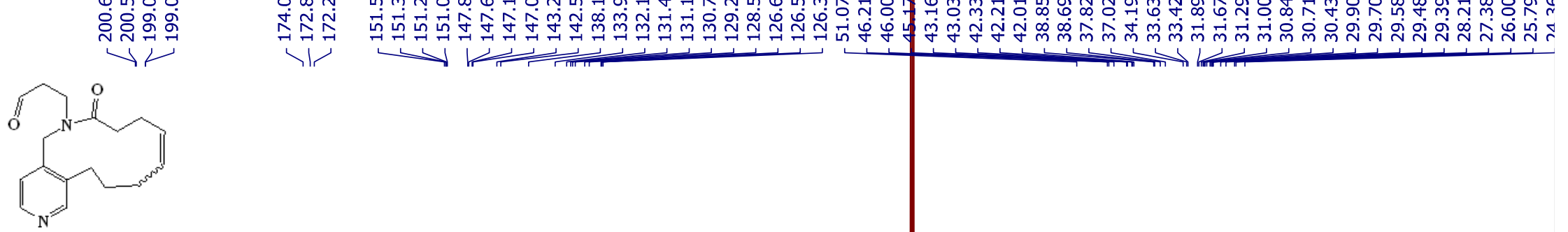

4

38

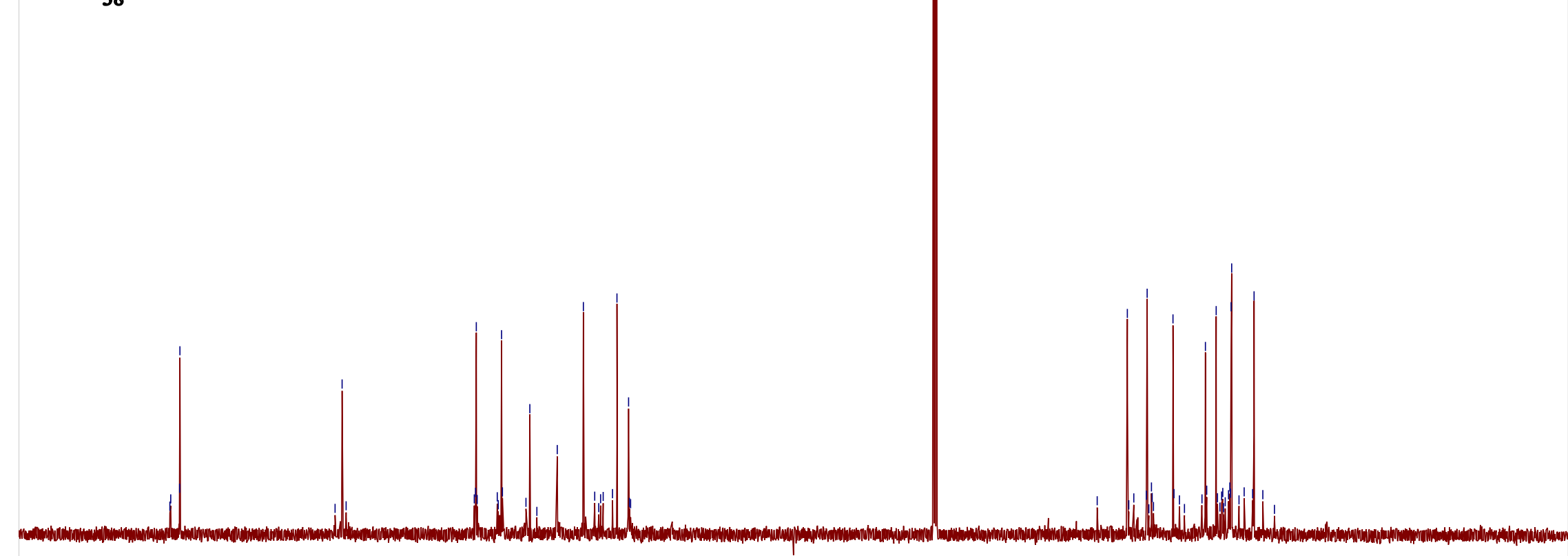

220

2102

$\begin{array}{lll}00 & 190 \quad 180\end{array}$

$170 \quad 160$

160150

$140 \quad 130$

120

$110 \underset{f 1(\mathrm{ppm})}{100} 90$

80

7060

50

40

3020

10

$\begin{array}{lll}0 & -10 & -20\end{array}$ 
AL-05-170.1.fid

1H AL-05-170

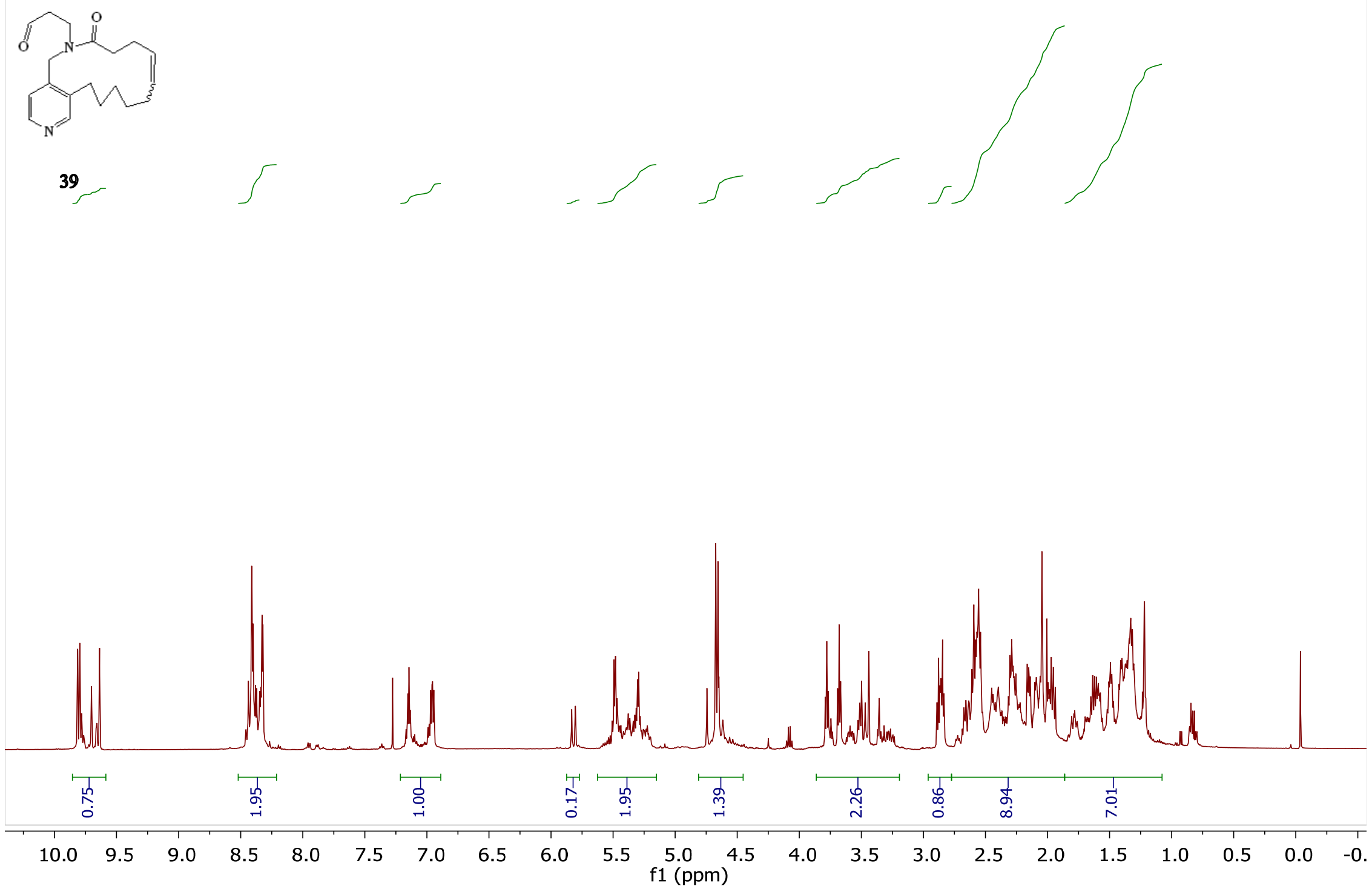




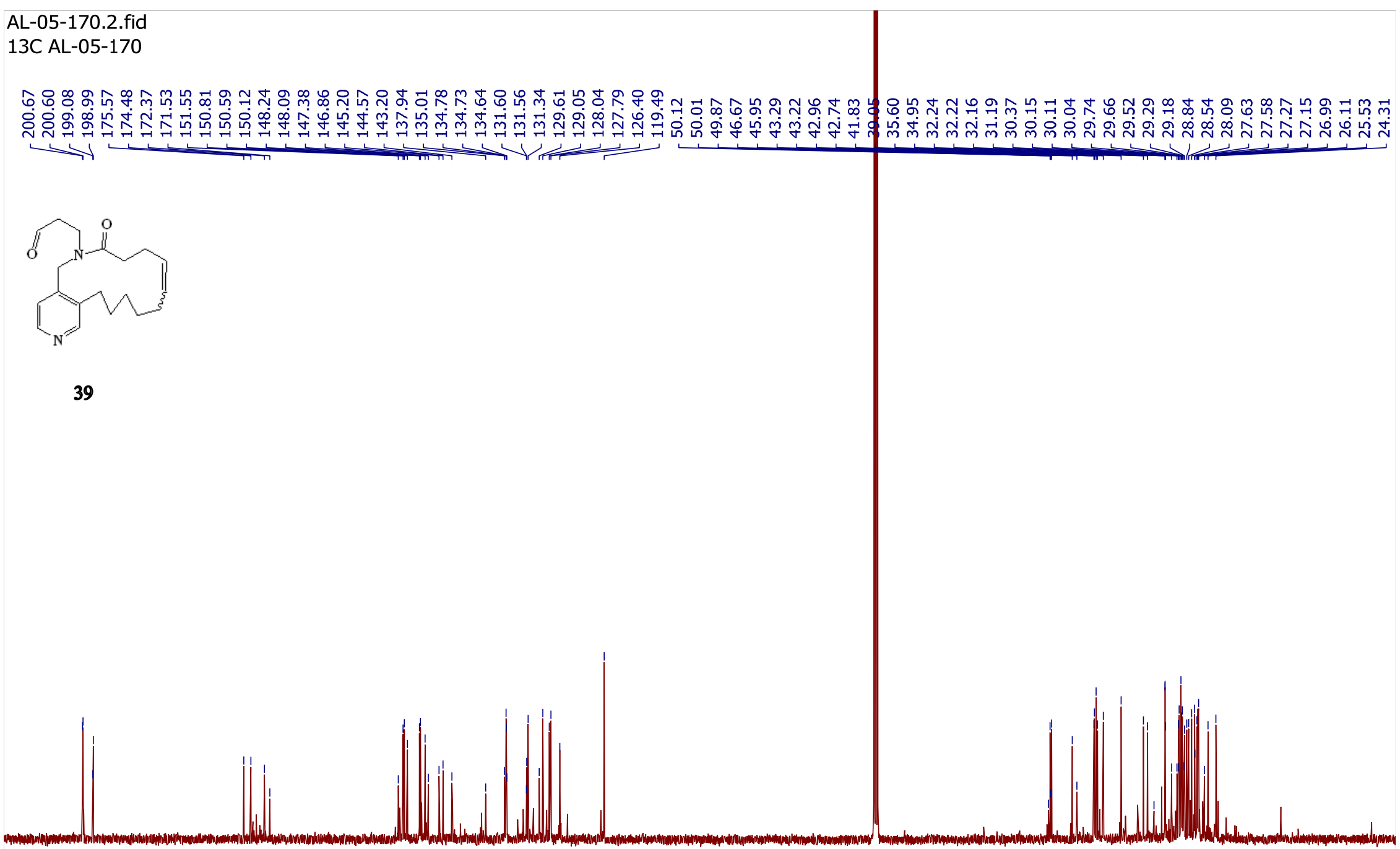

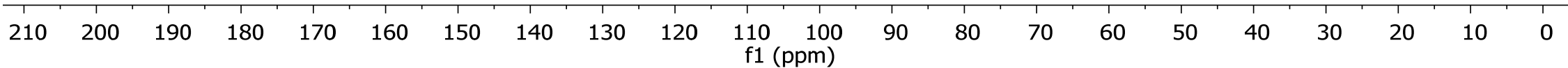


AL-06-86.1.fid

1H AL-06-86
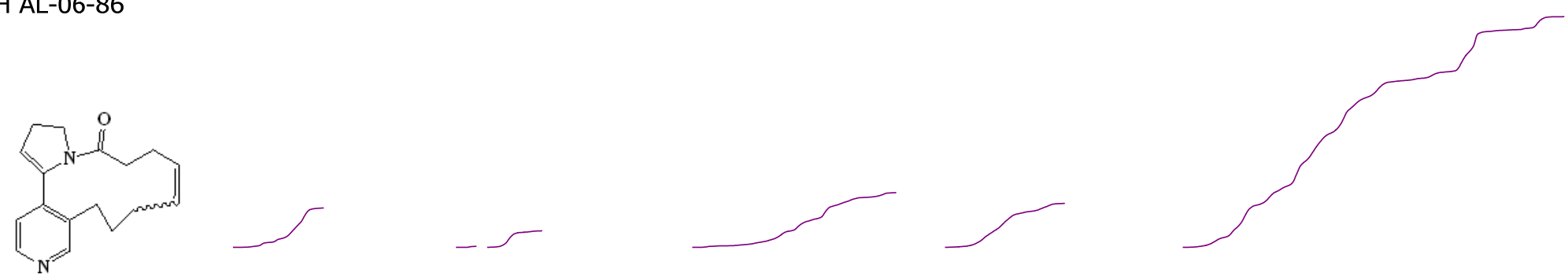

40

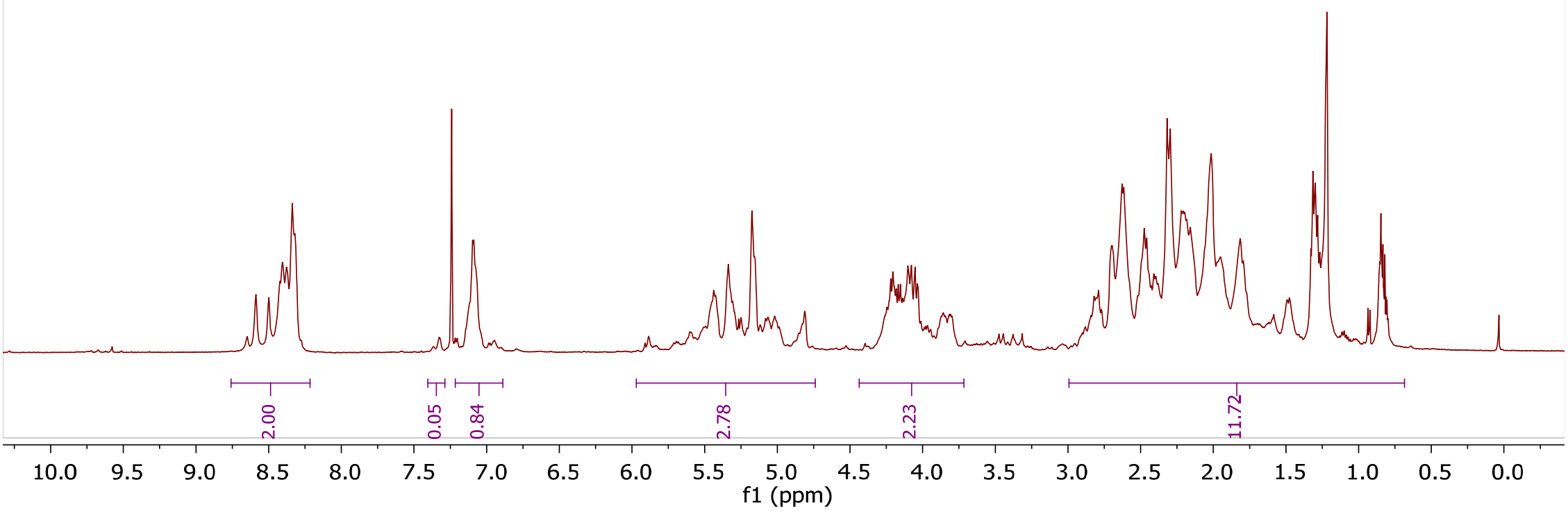


AL-06-86.2.fid

$13 \mathrm{C}$

Nov 302012

Santhana

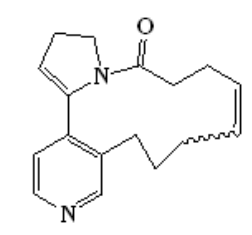

40

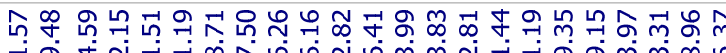

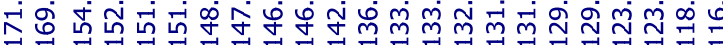

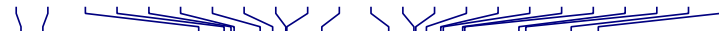

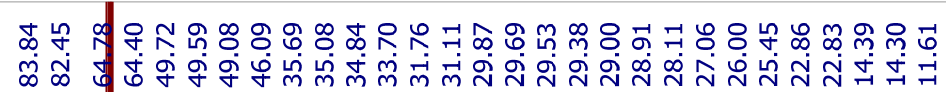

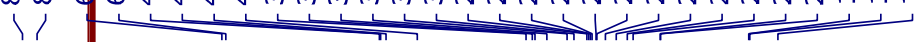

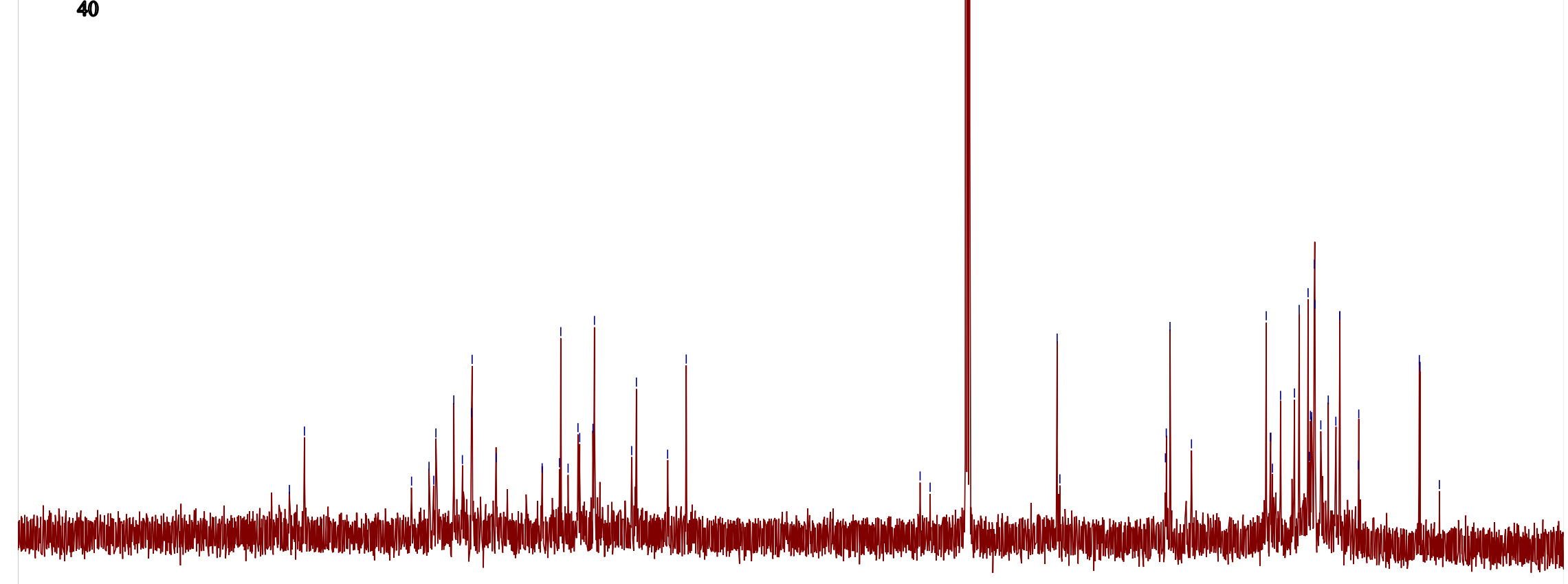

200190

$180 \quad 170 \quad 160$

150

140

130

120

$\begin{array}{ll}110 & 100 \\ \mathrm{f} 1 & (\mathrm{ppm})\end{array}$

90

80

70

60

$50 \quad 40$

30

20

100 
AL-05-226 Spot 1.1.fid

1H AL-05-226 Spot 1
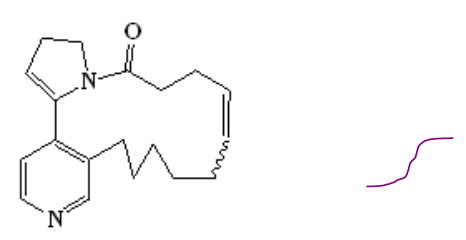

41
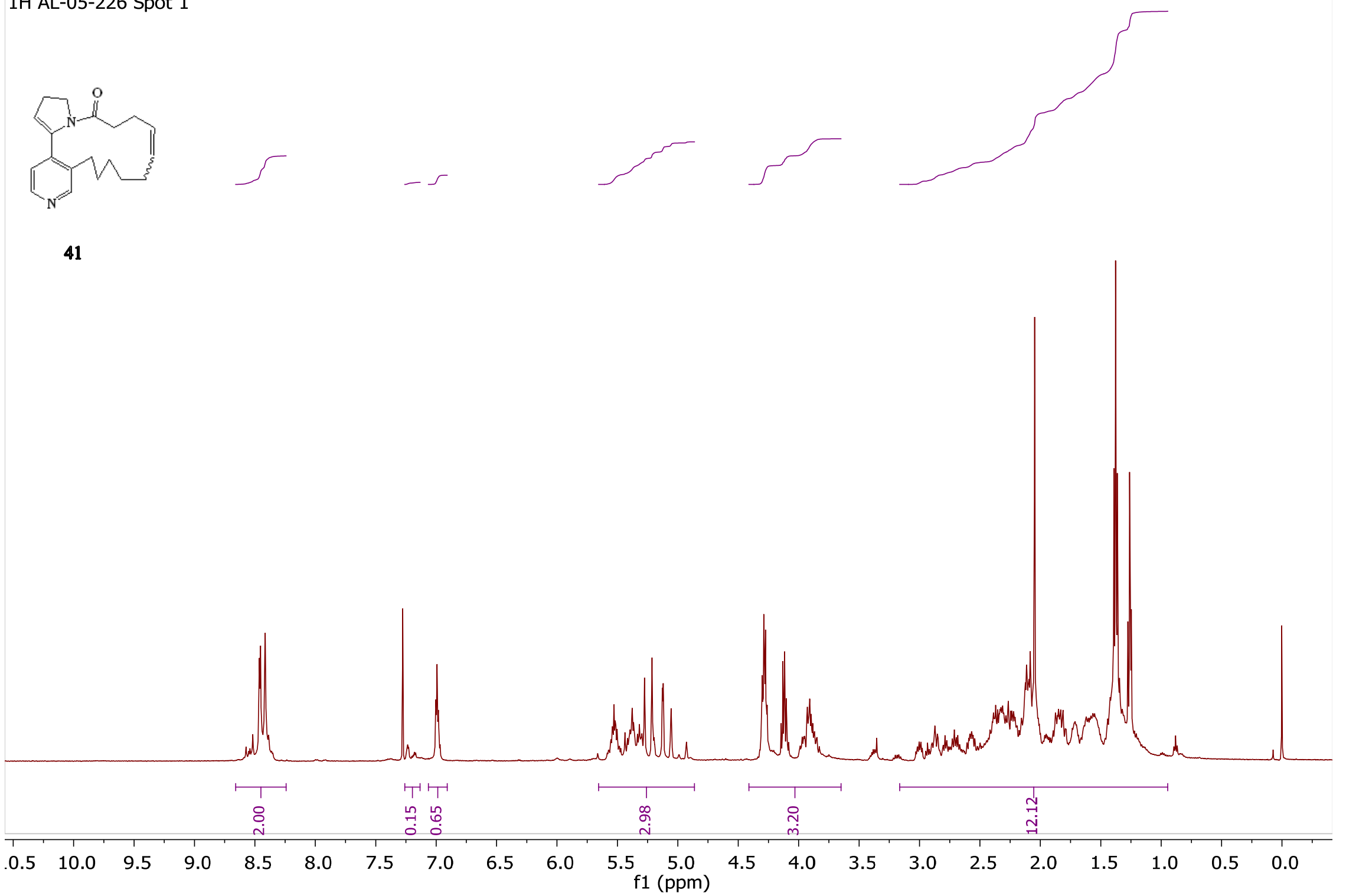


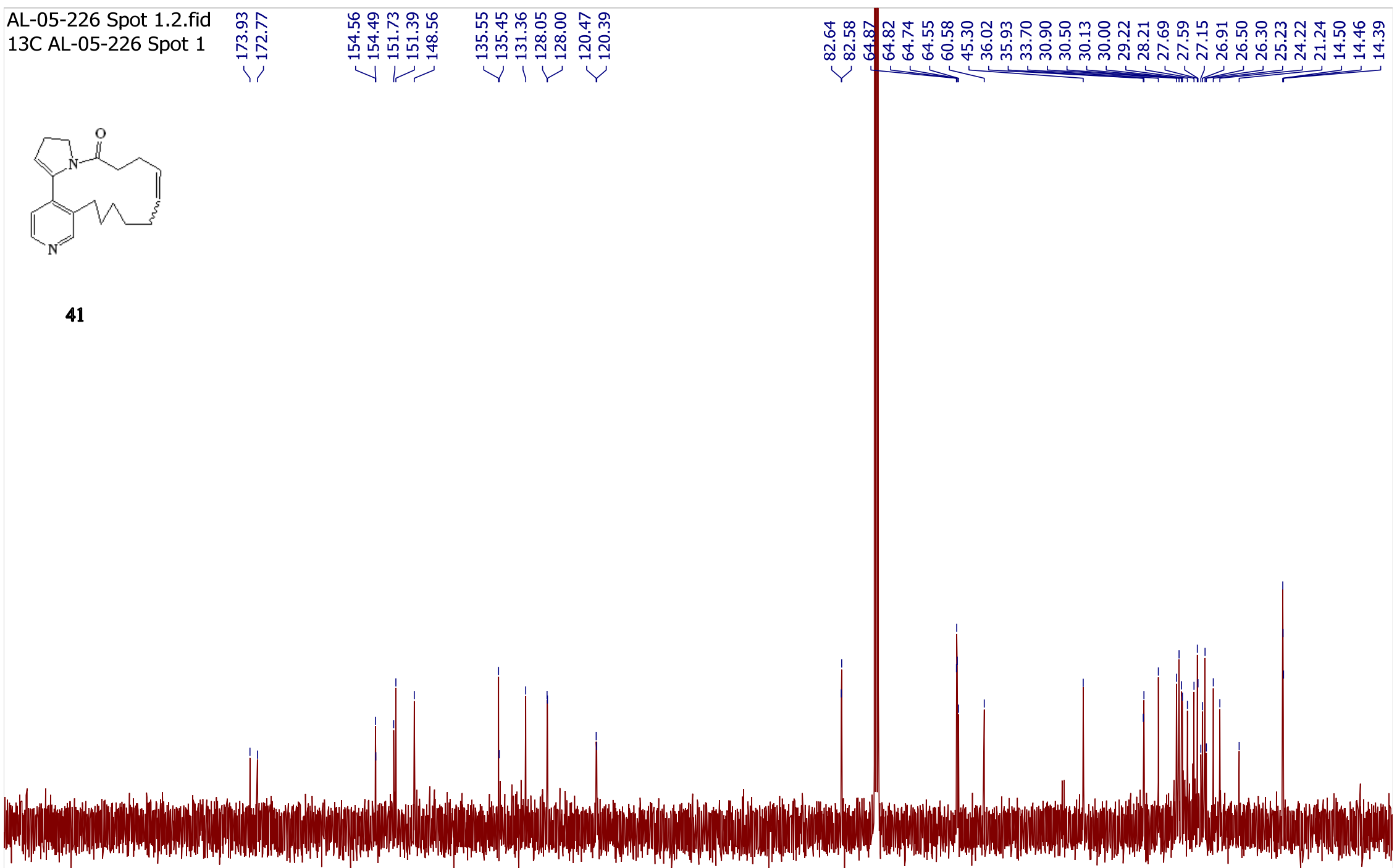

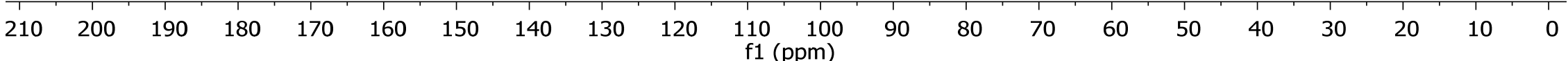




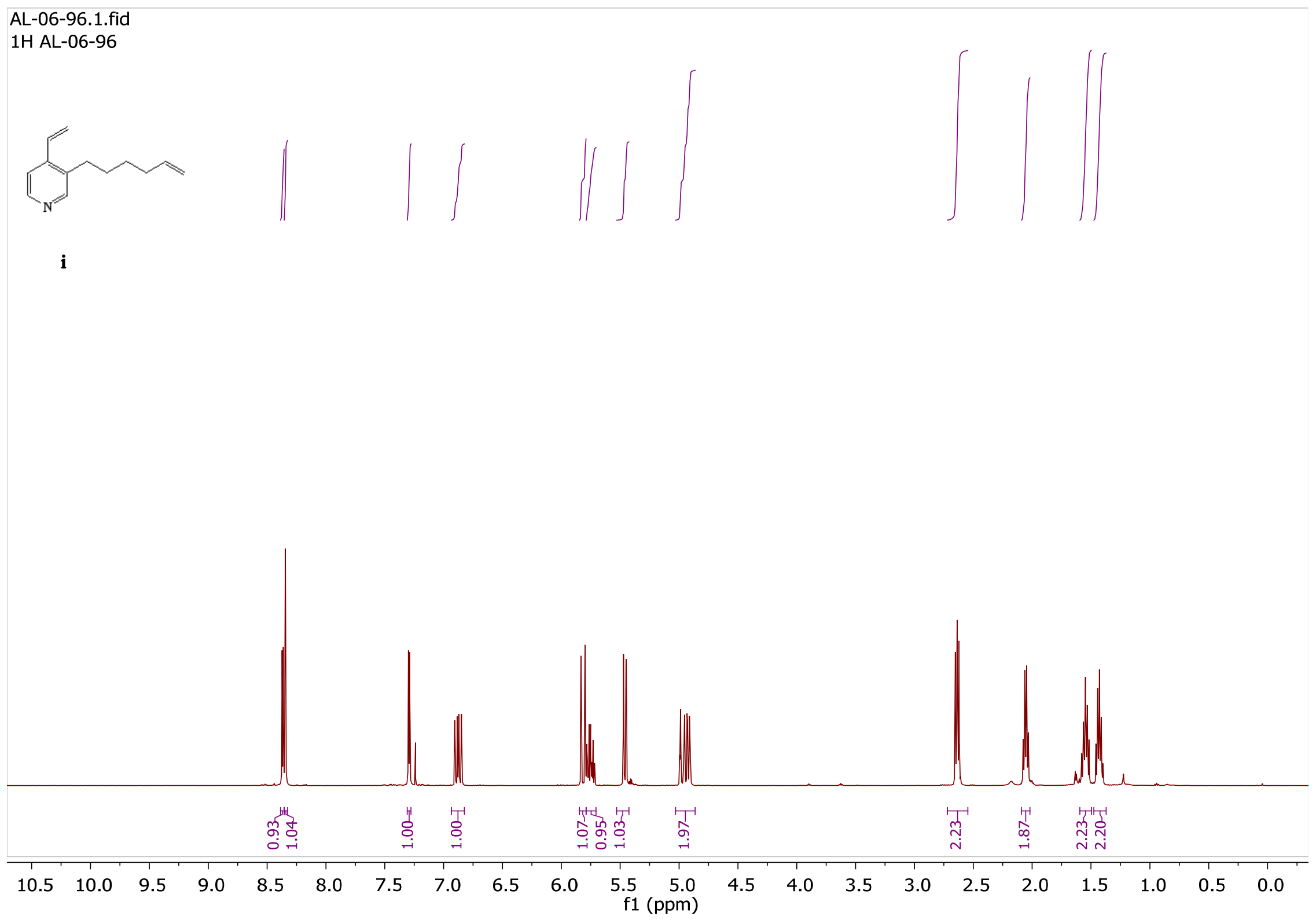

S61 


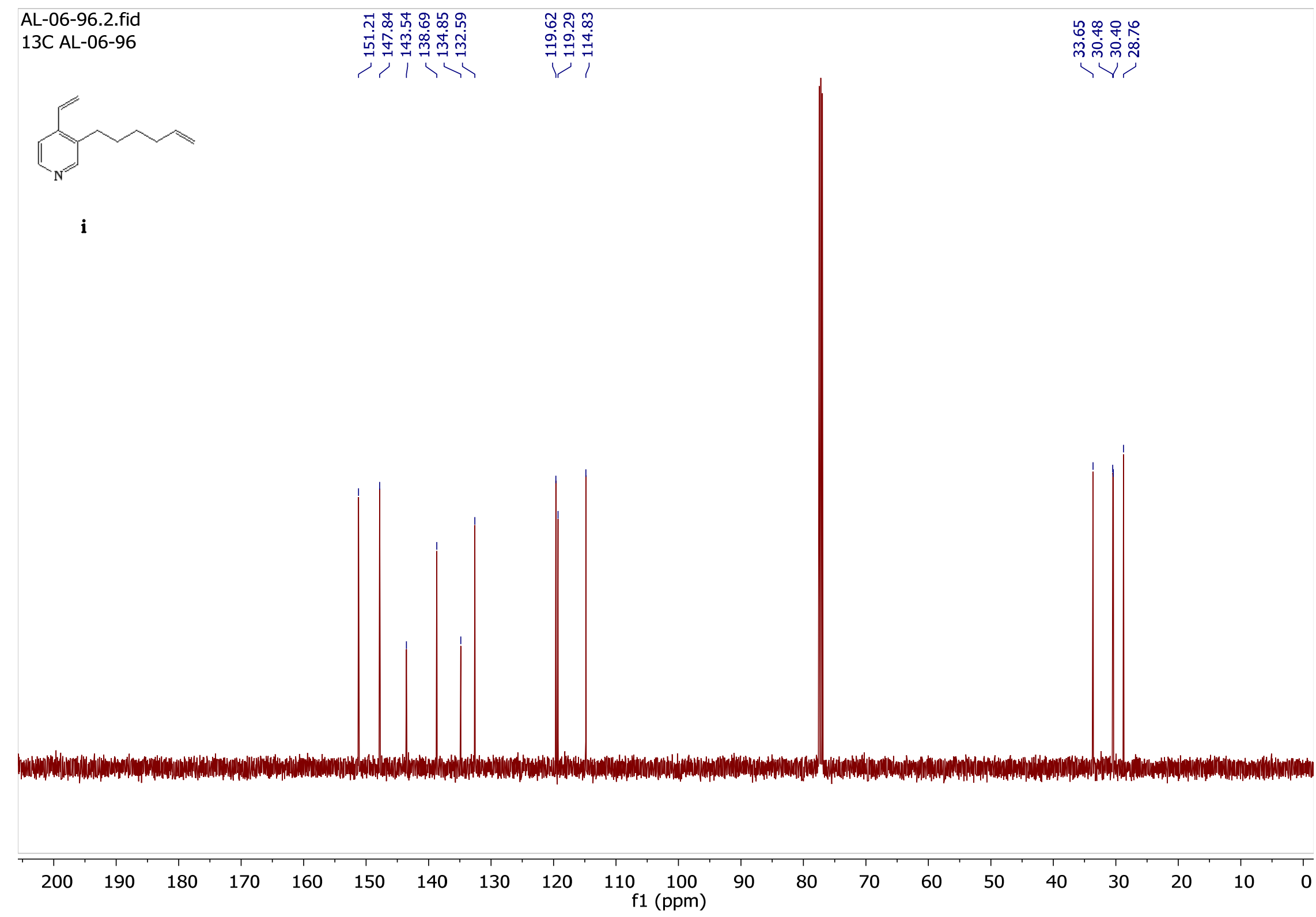


AL-06-102.1.fid

1H AL-06-102
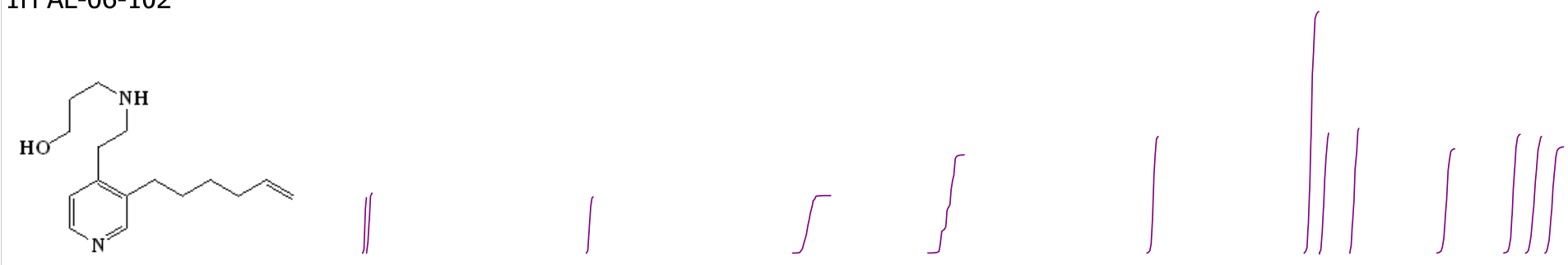

42

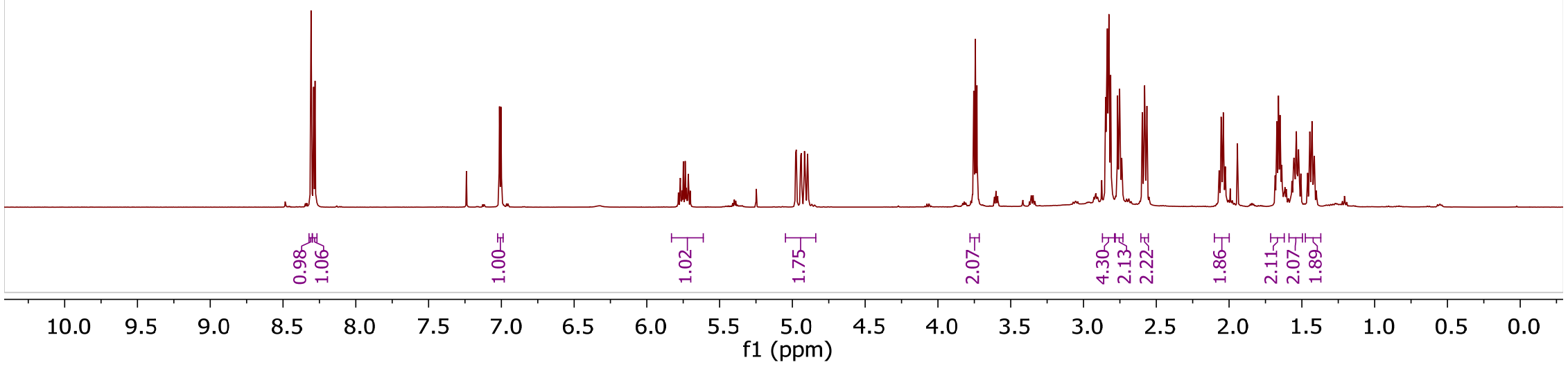




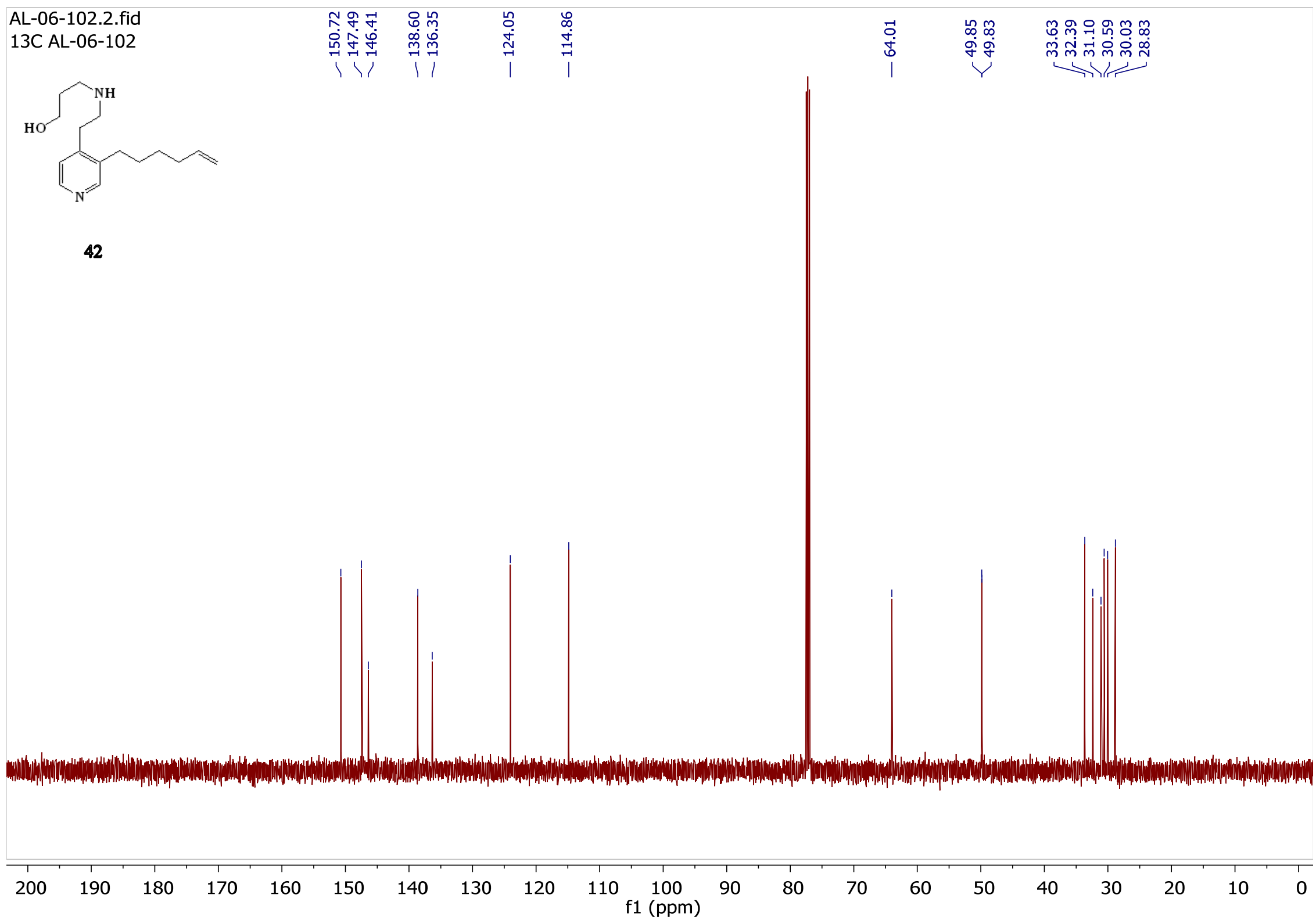




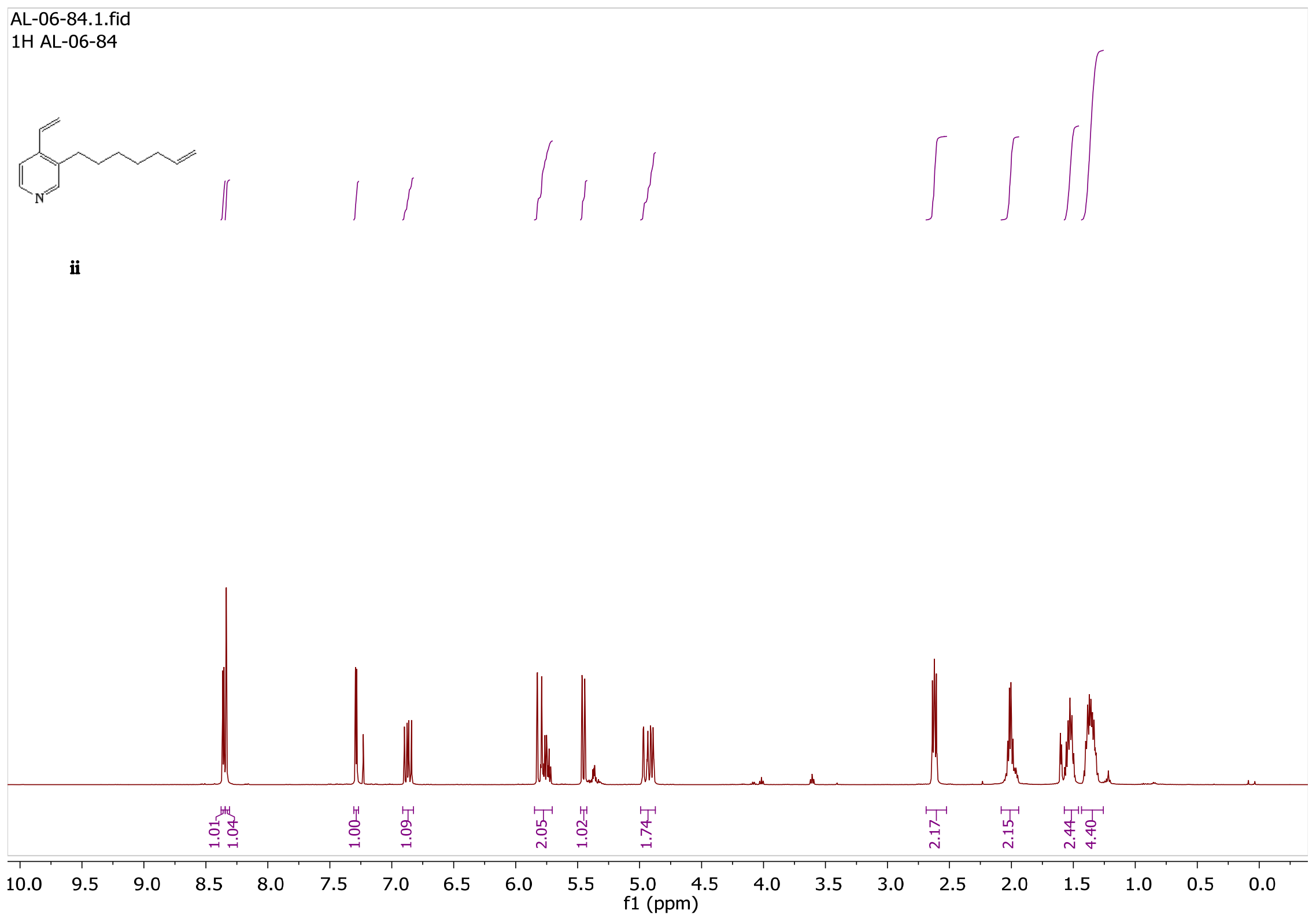

S65 
AL-06-84.2.fid

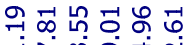

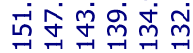

ભํํ요

芯的员む范

13C AL-06-84

न)। न्न

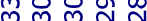

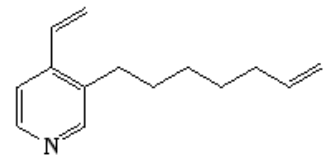

ii

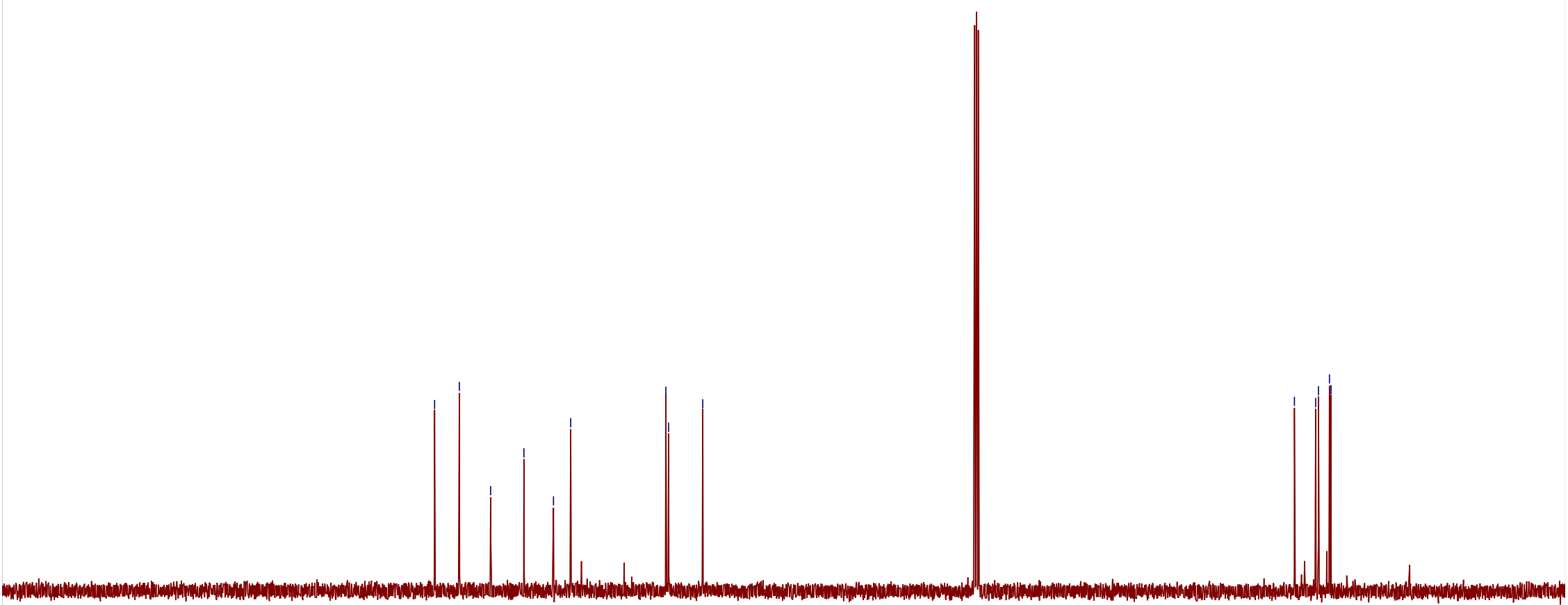

$\begin{array}{llll}10 & 200 & 190 & 180\end{array}$

$\begin{array}{llll}170 & 160 & 150 & 140\end{array}$

130

120

$110 \quad 100$

90

80

70

60

50

40

30

20

100 


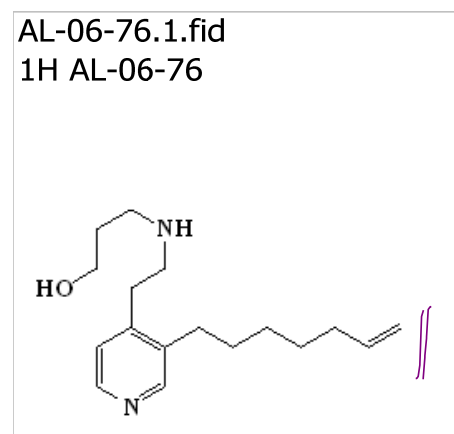

43

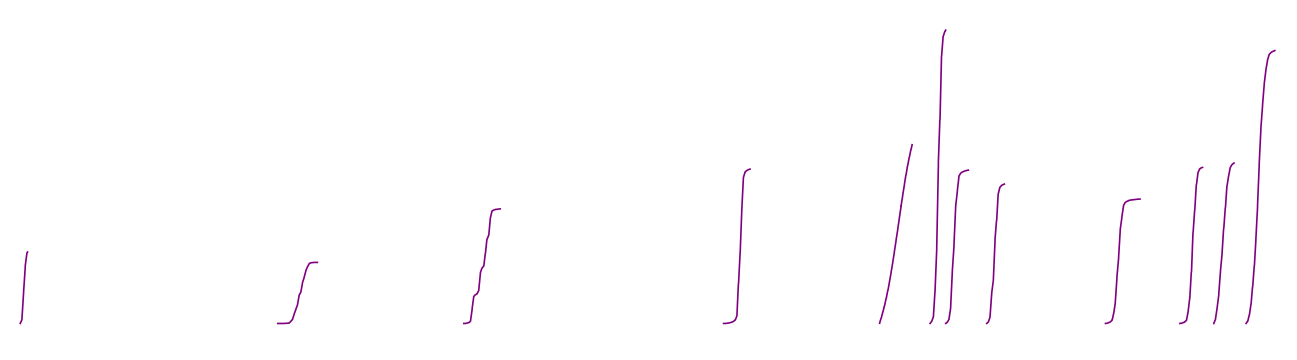




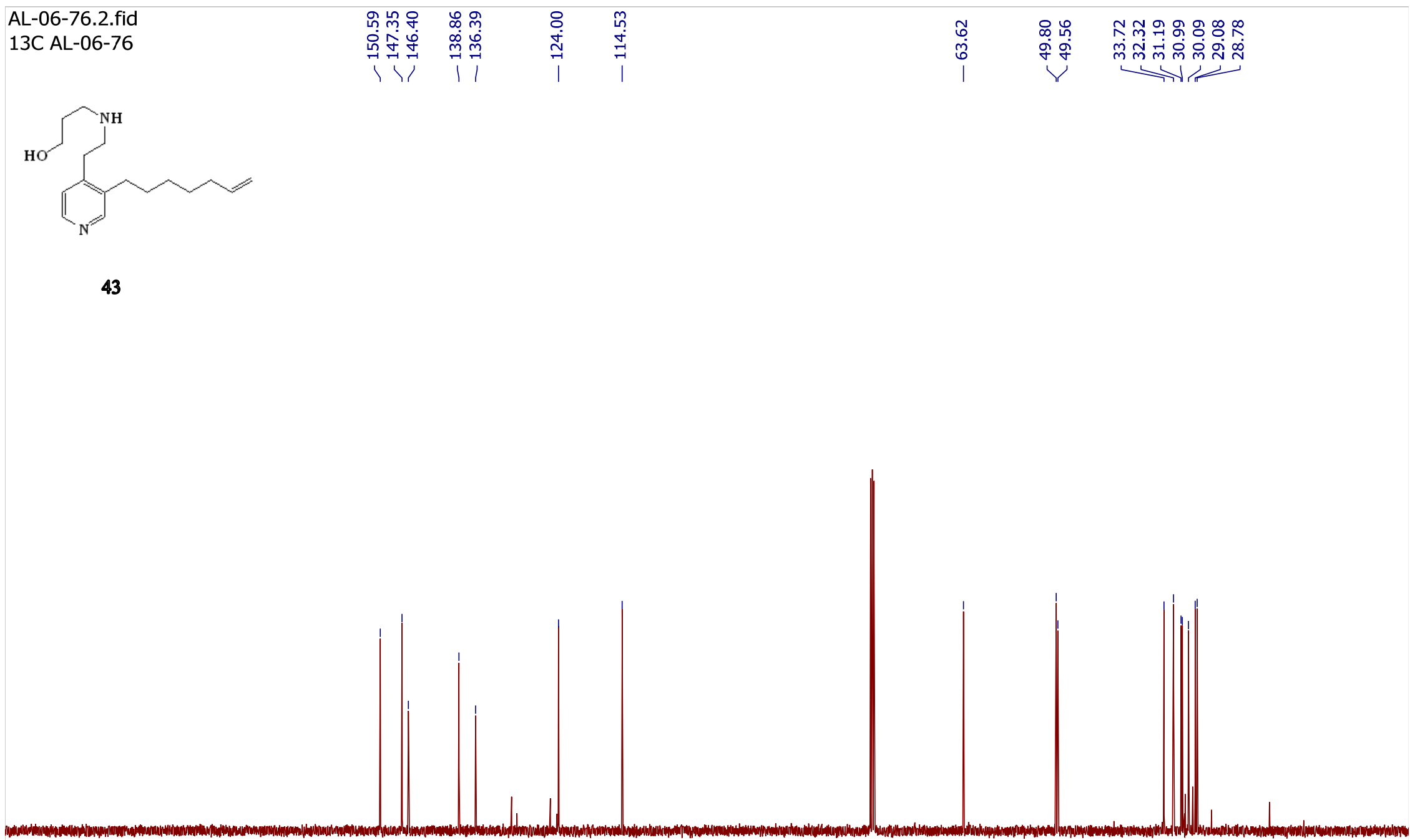

$\begin{array}{lllllllllllllllllllllll}200 & 190 & 180 & 170 & 160 & 150 & 140 & 130 & 120 & 110 & \begin{array}{l}100 \\ \mathrm{f} 1(\mathrm{ppm})\end{array} & 90 & 80 & 70 & 60 & 50 & 40 & 30 & 20 & 10 & 0\end{array}$




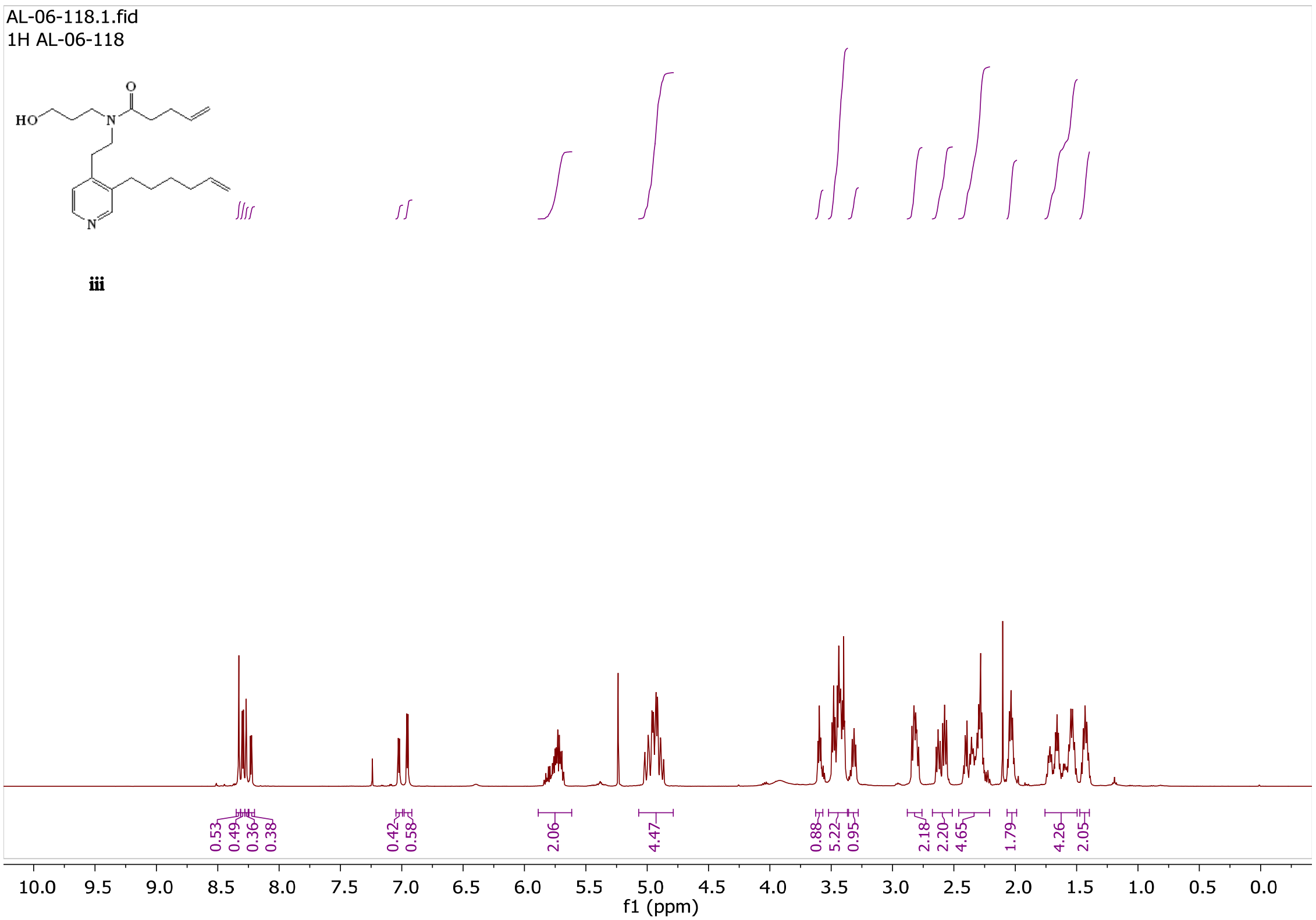




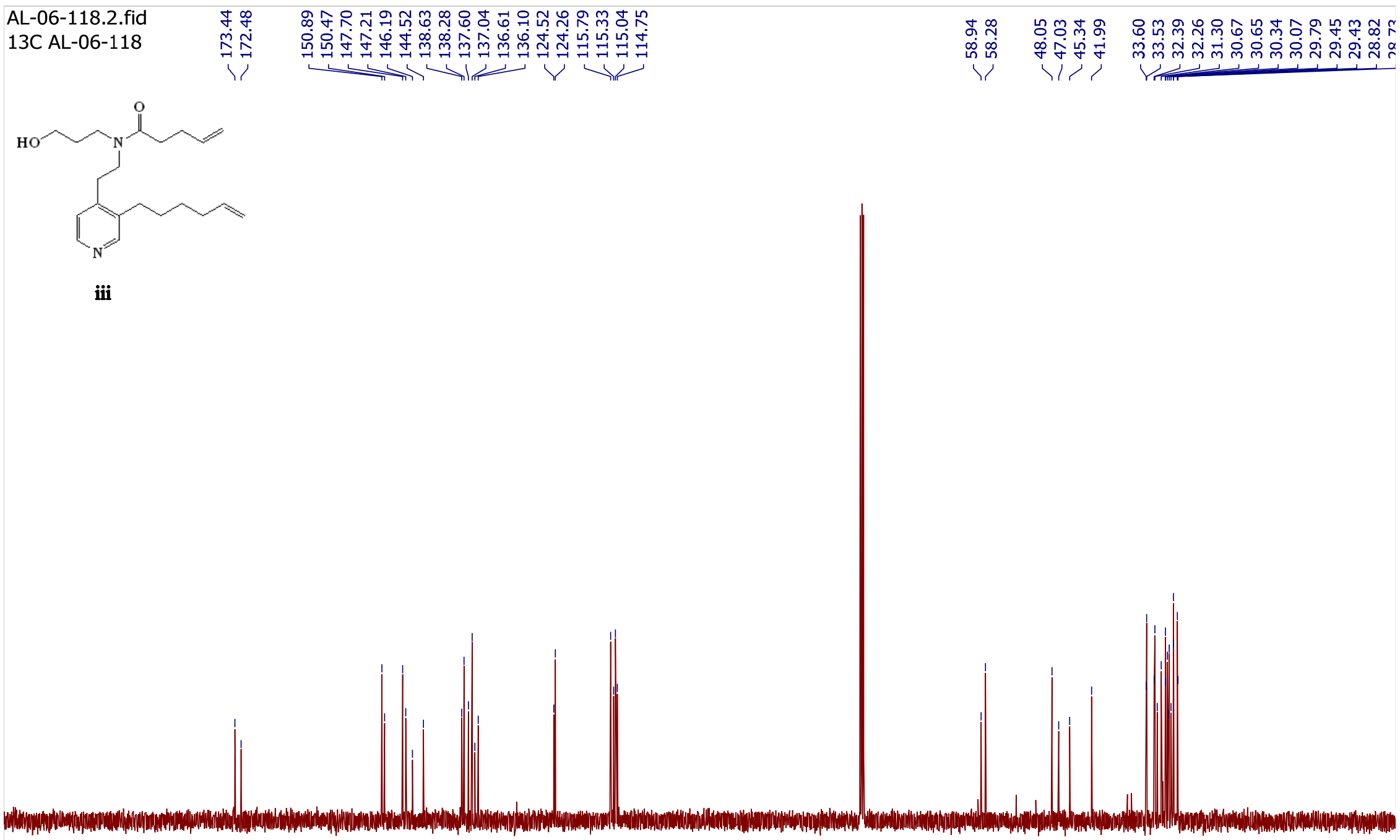

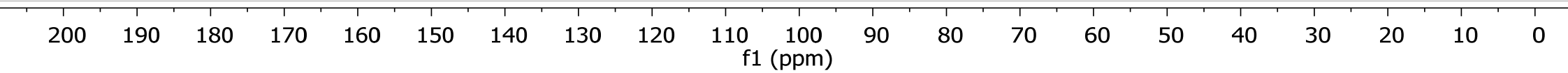




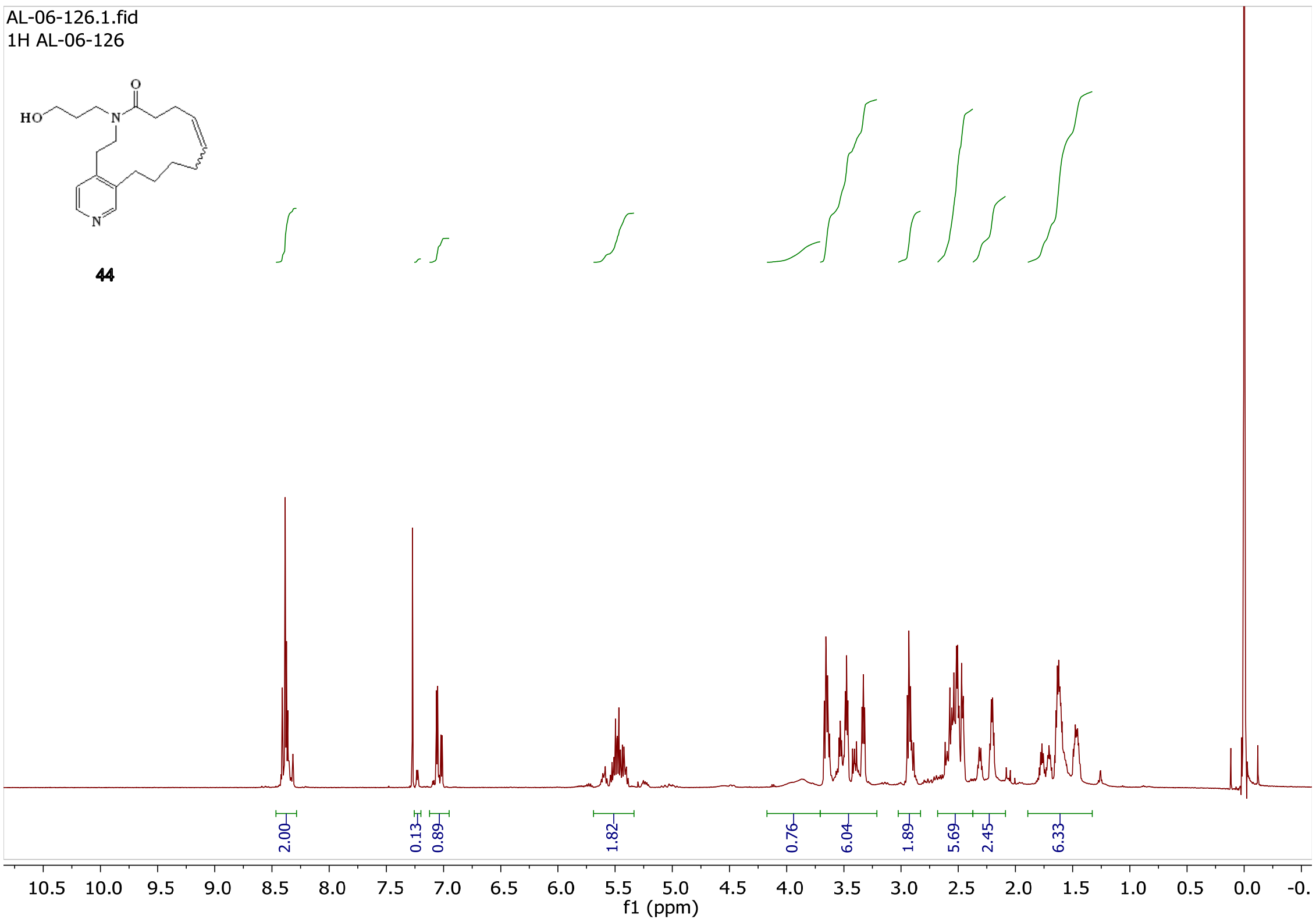




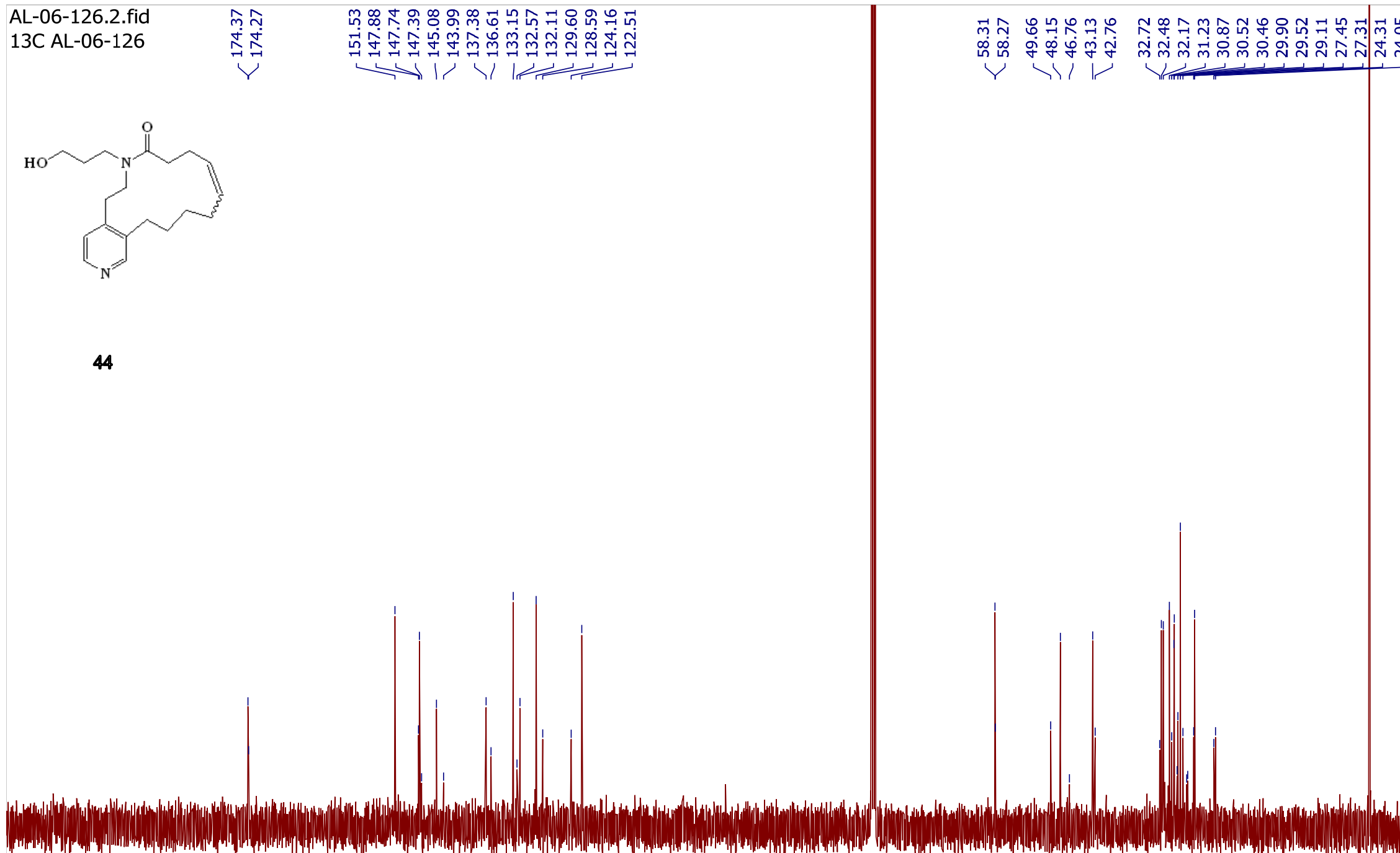

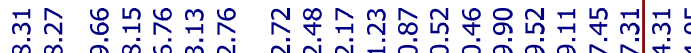

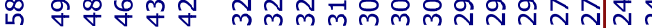

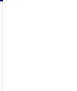


AL-06-78.1.fid

1H AL-06-78
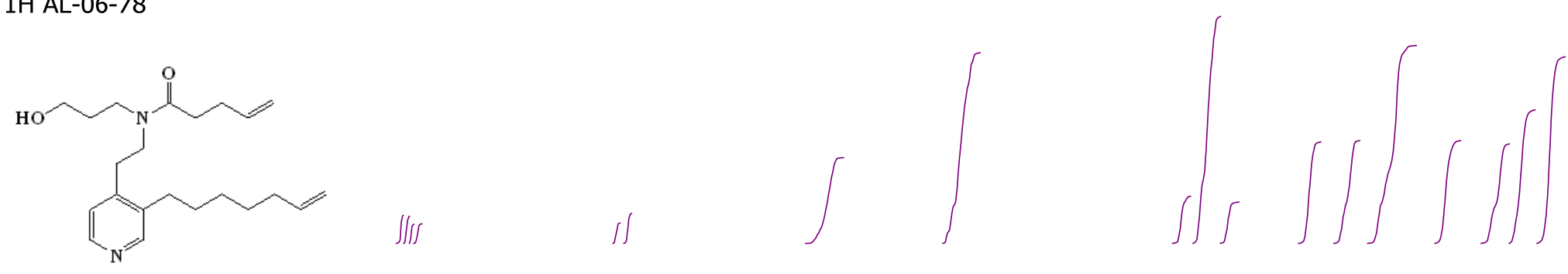

iv

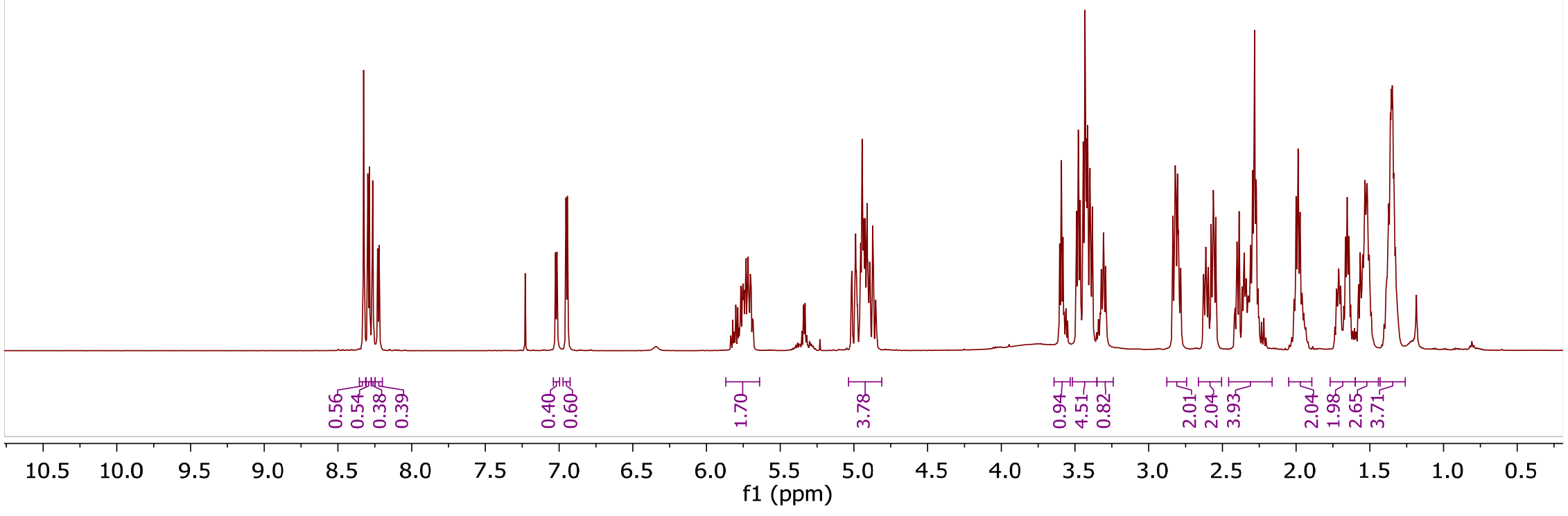




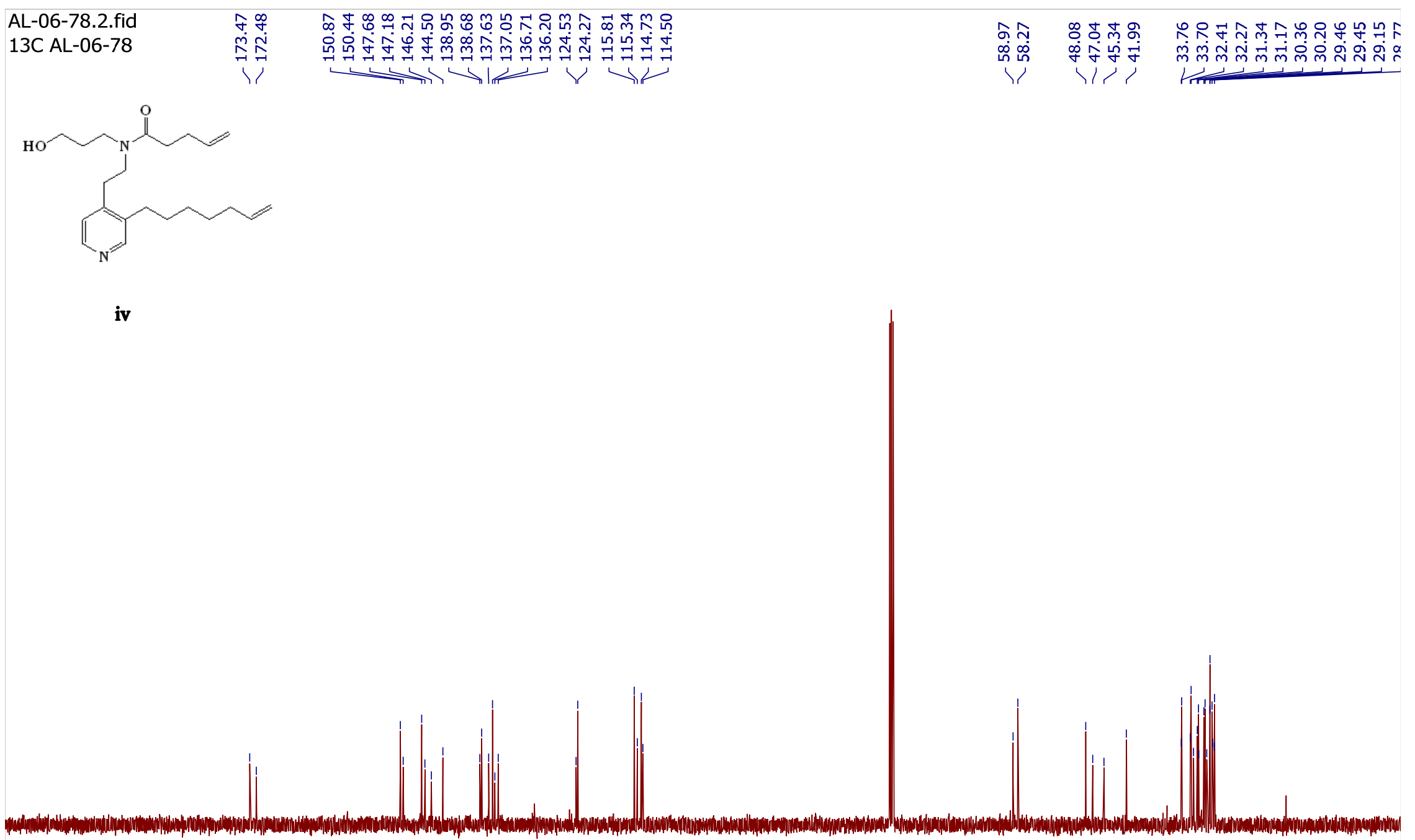

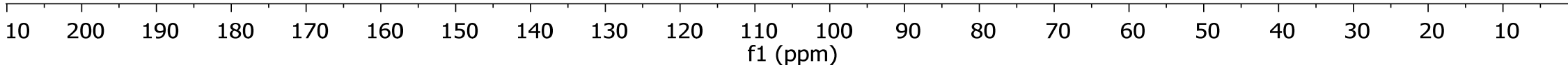


AL-06-82.1.fid

1H AL-06-82
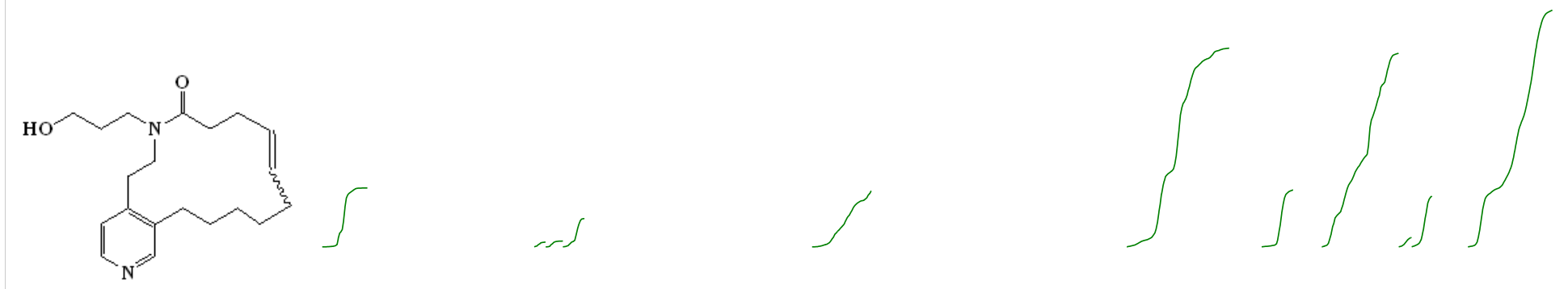

45

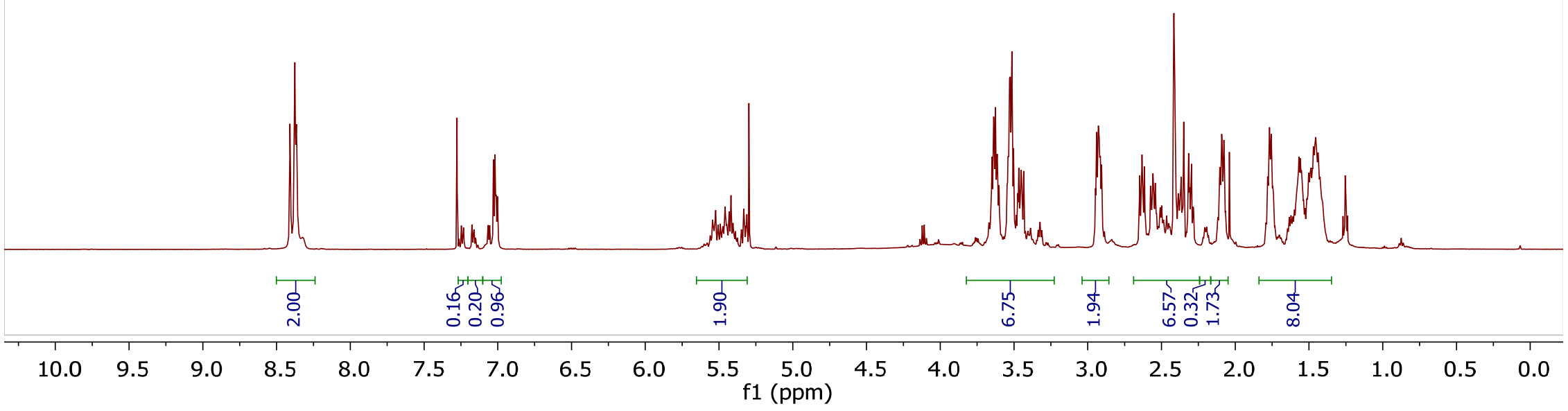




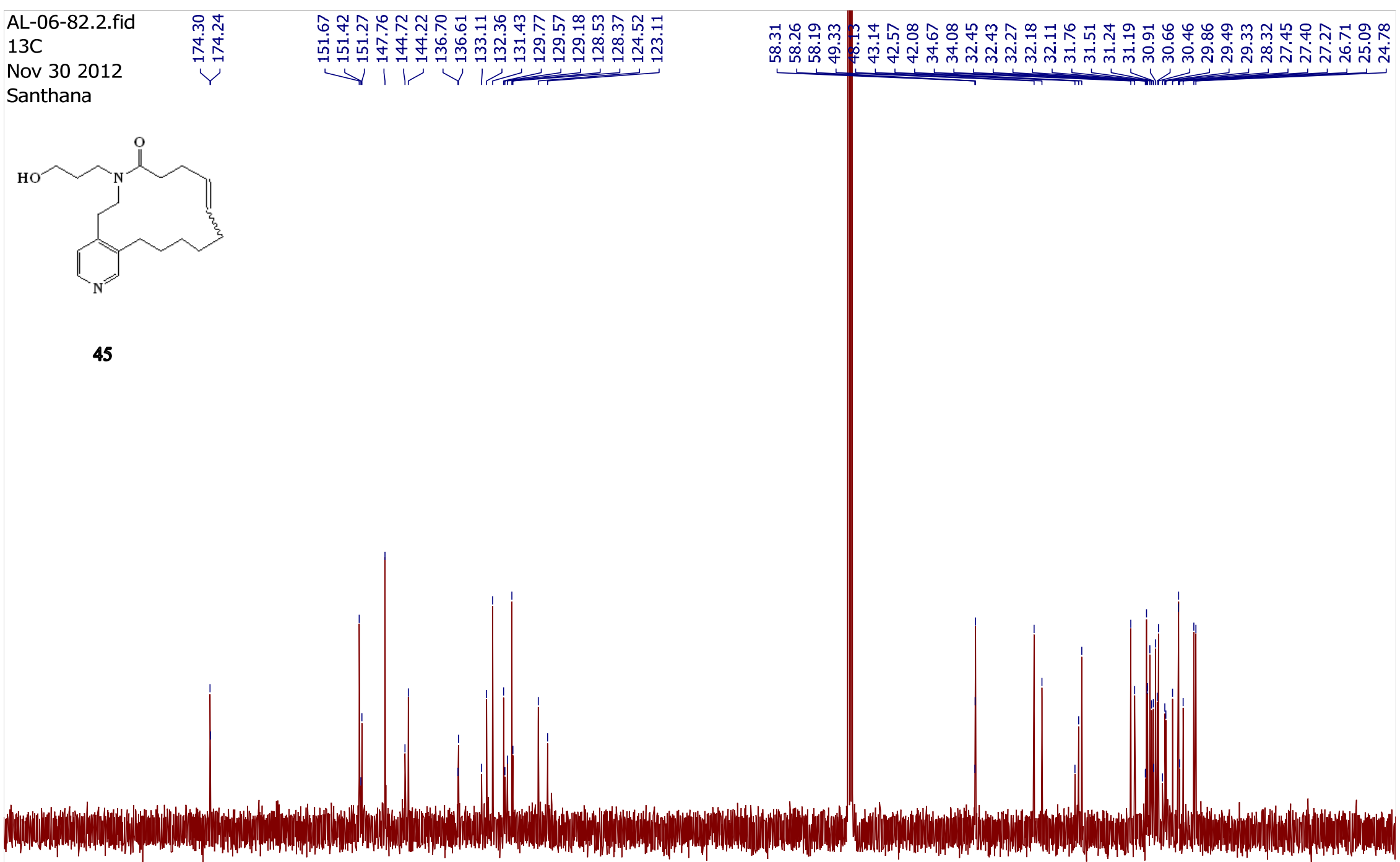

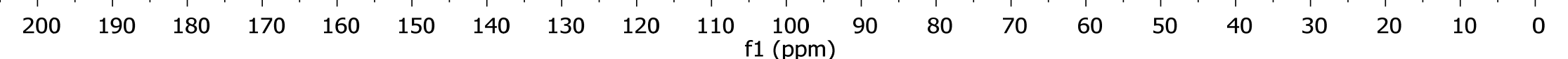


AL-06-184.1.fid

1H AL-06-184

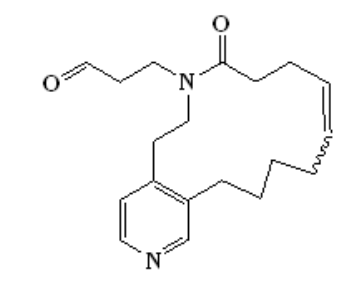

46
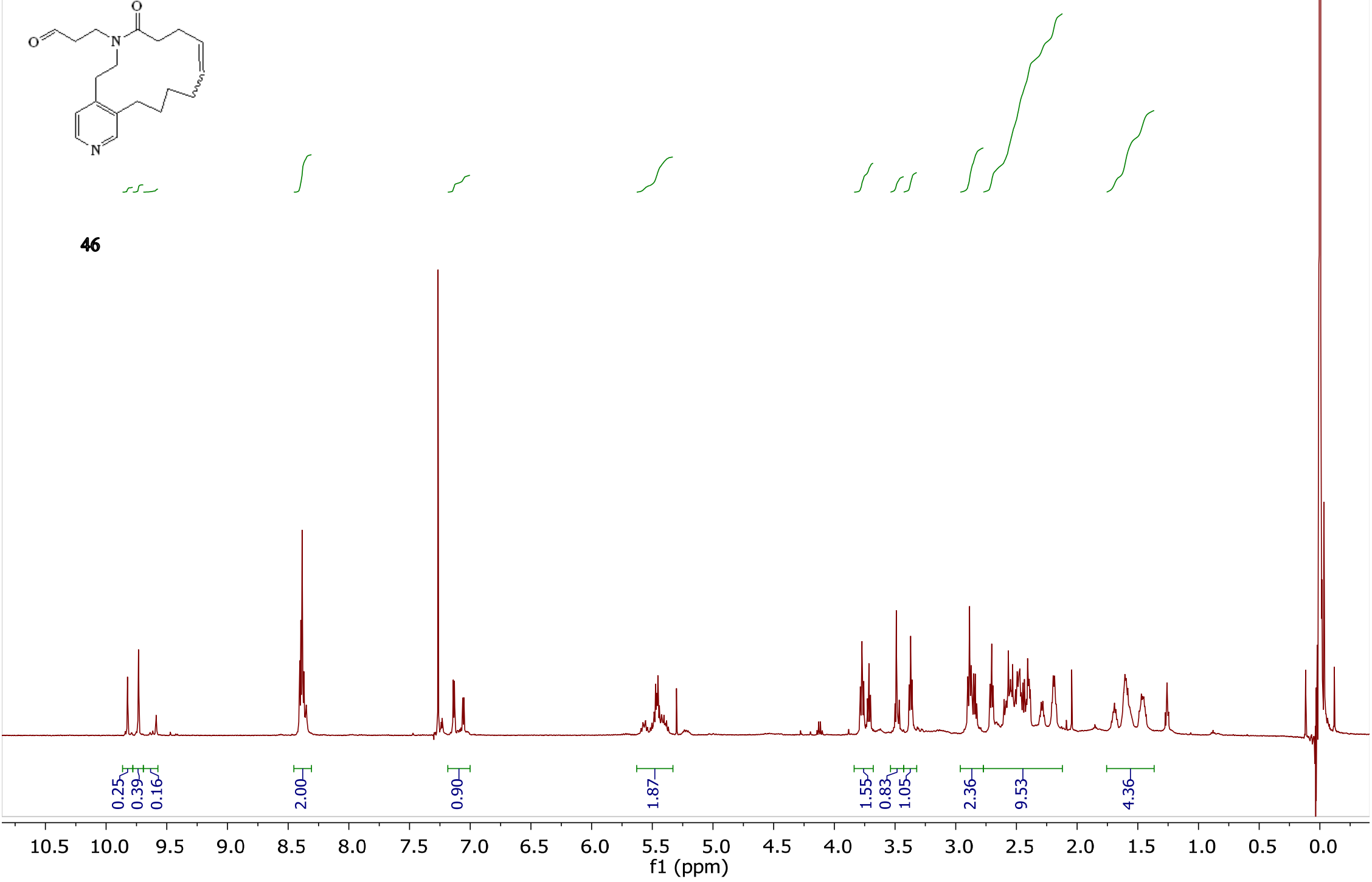


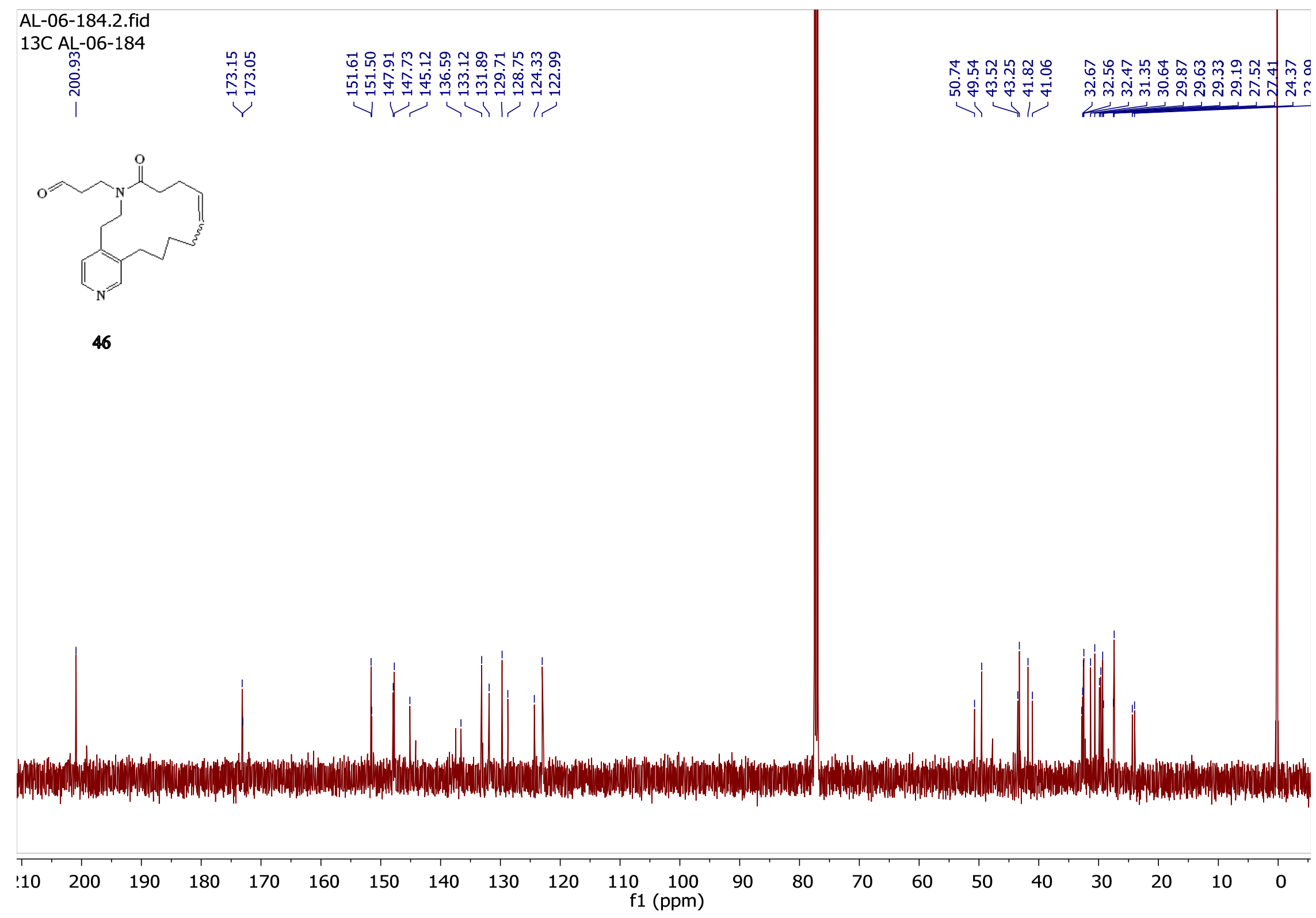


AL-06-128.1.fid

1H AL-06-128

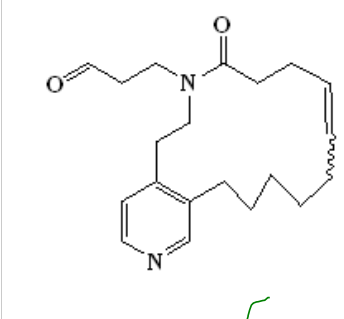

47
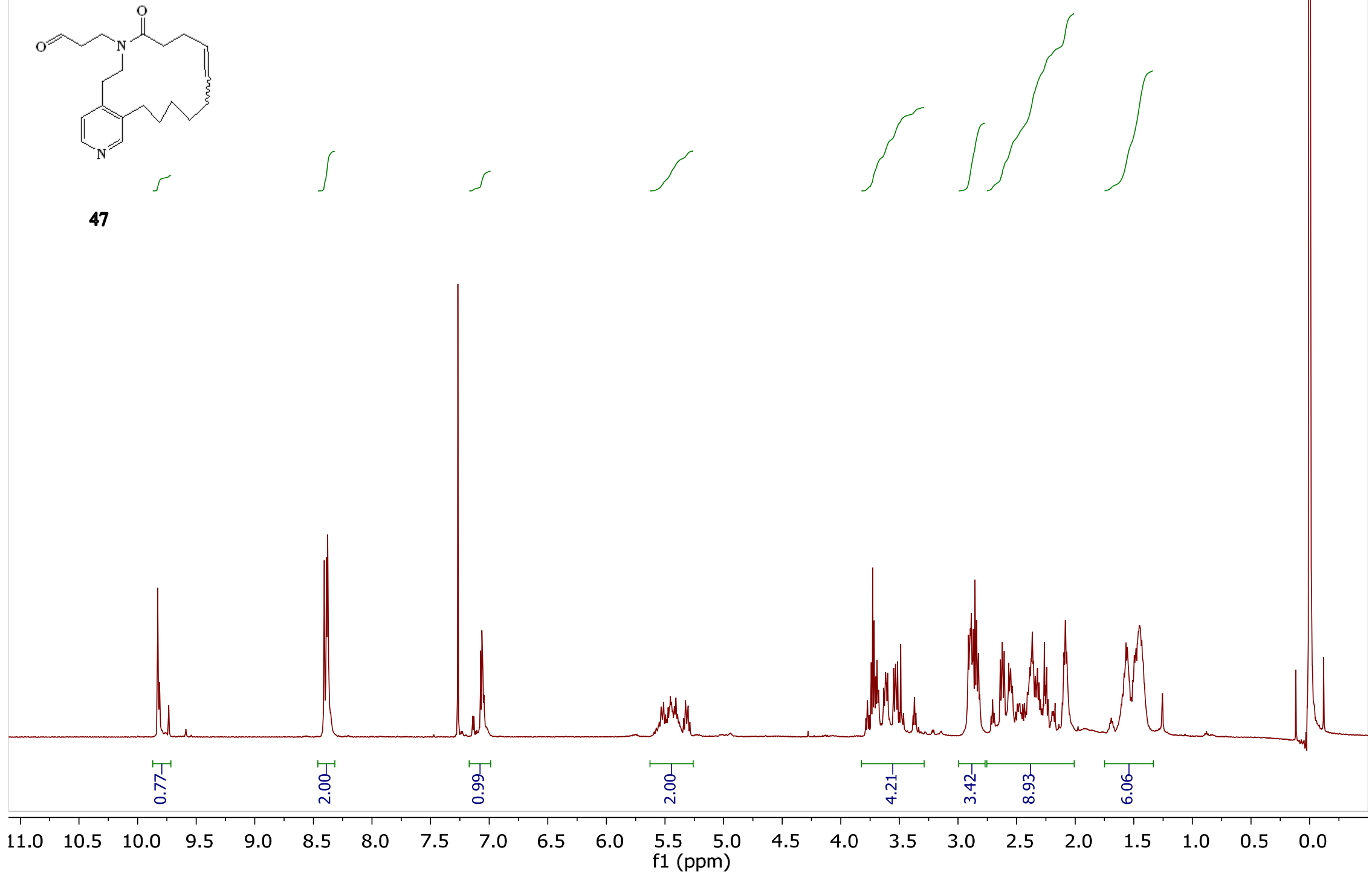


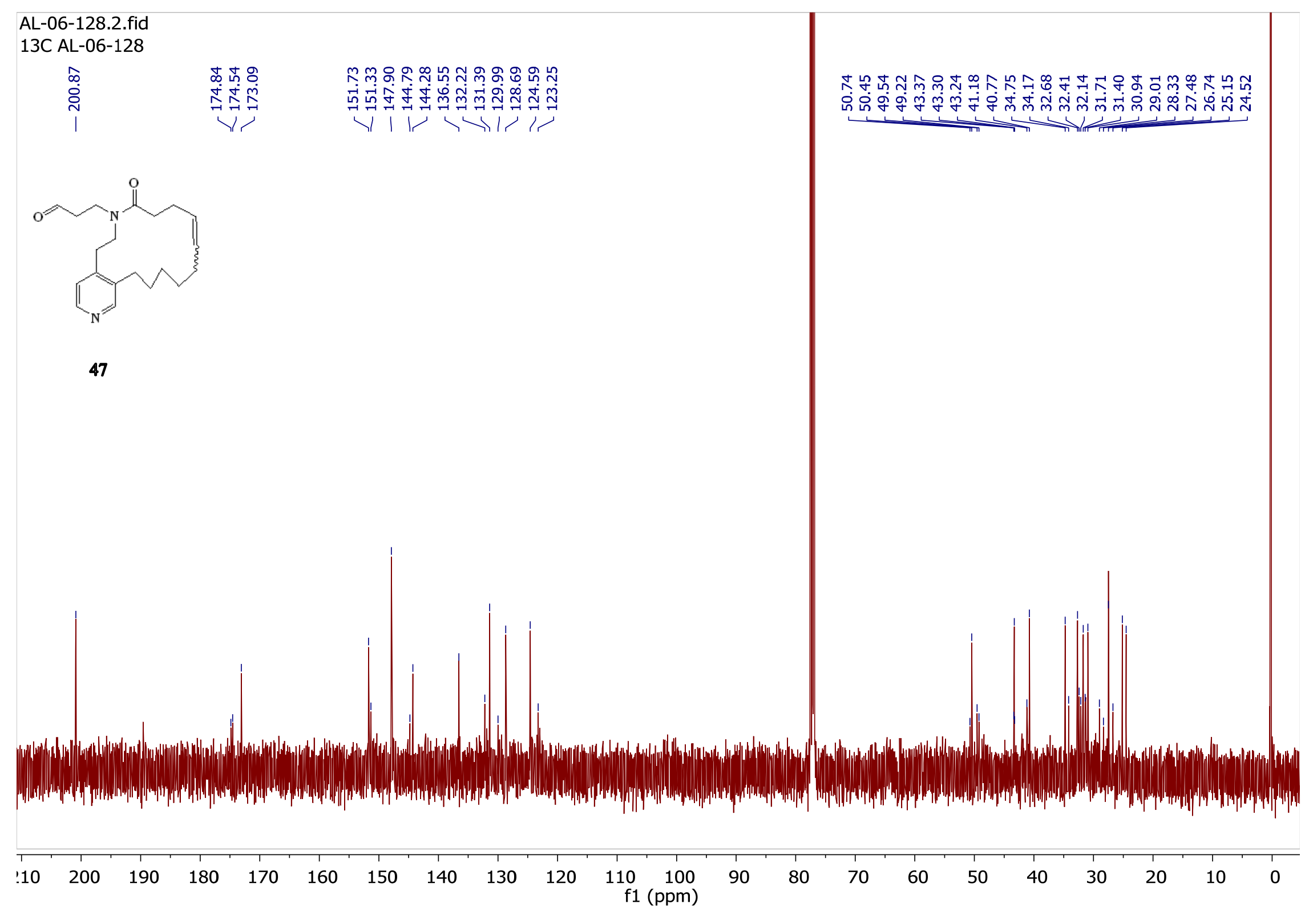


AL-05-186/1

1H AL-05-186
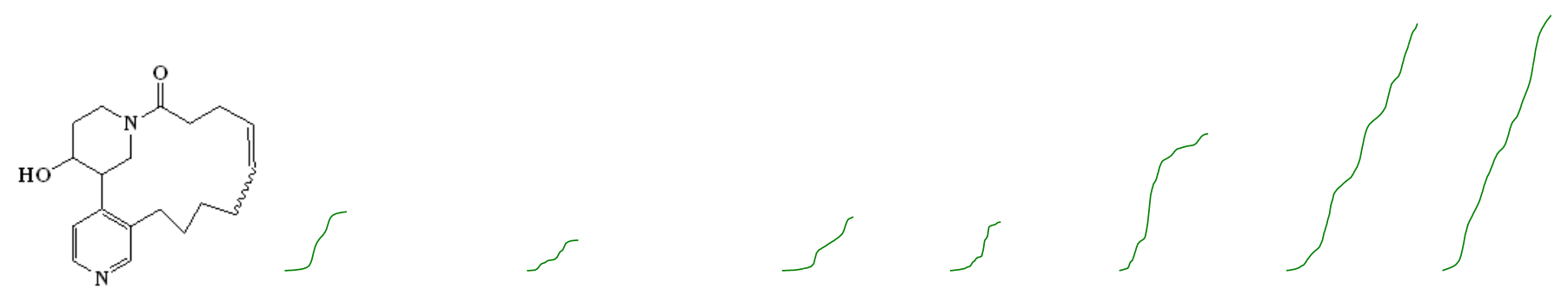

48

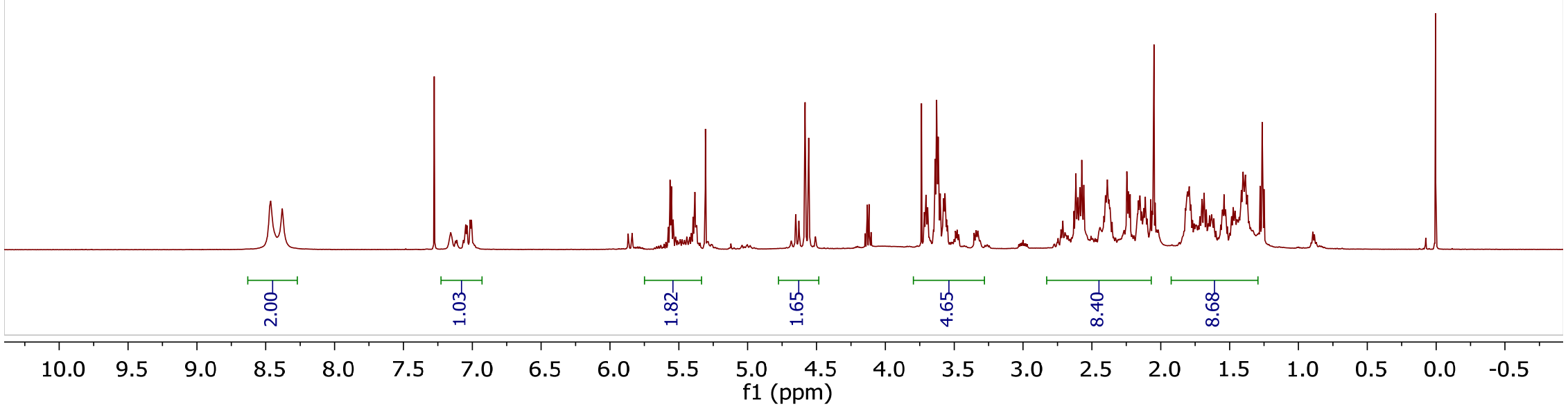


AL-06-130.1.fid

$1 \mathrm{H} \mathrm{AL-06-130}$
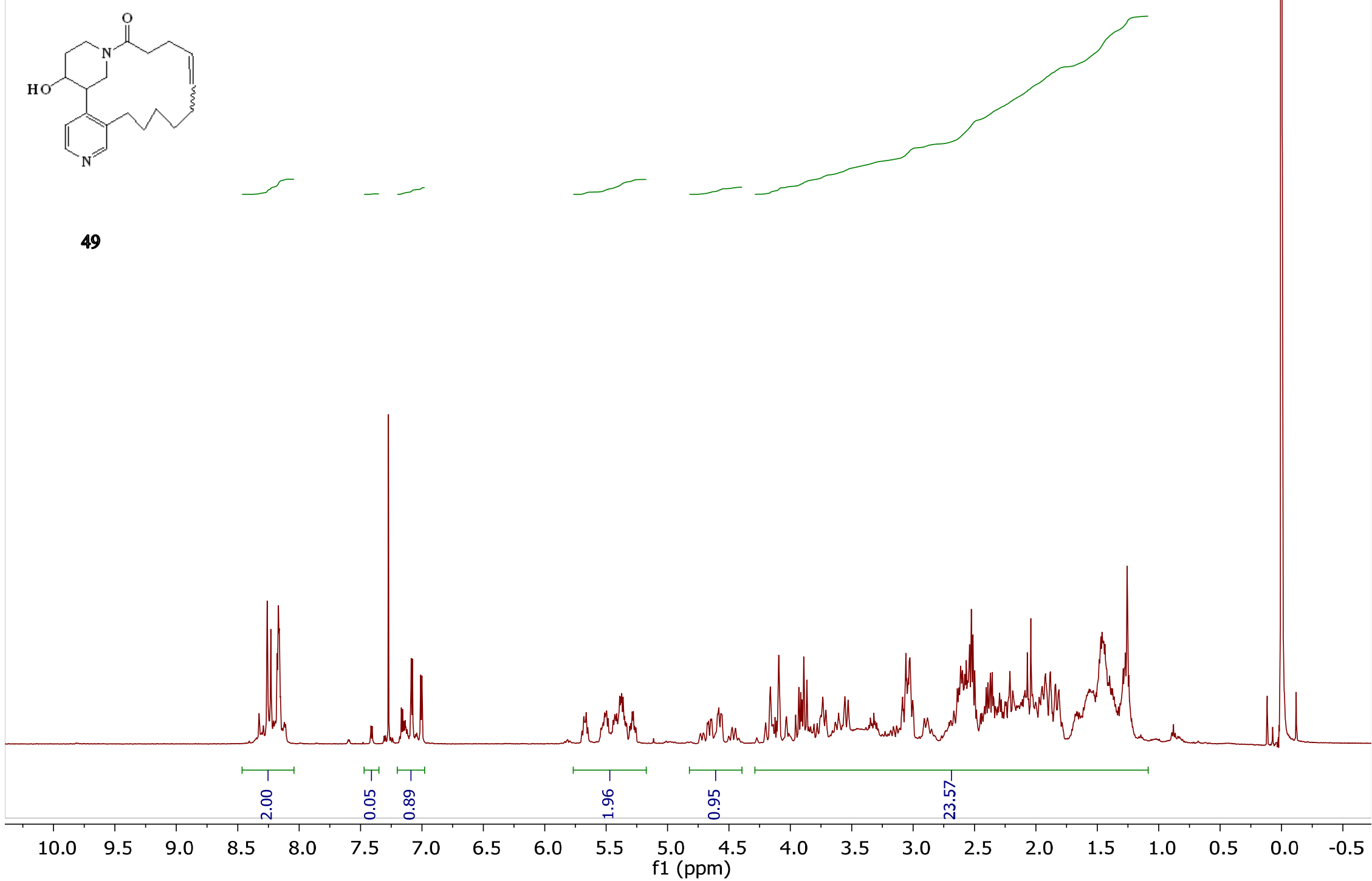
AL-06-130.2.fid

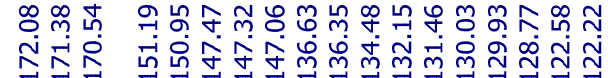

13C AL-06-130

(i)

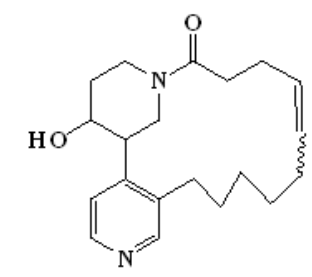

49

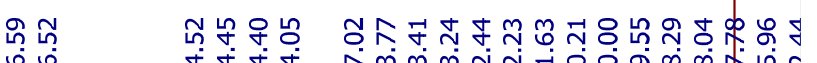

பี

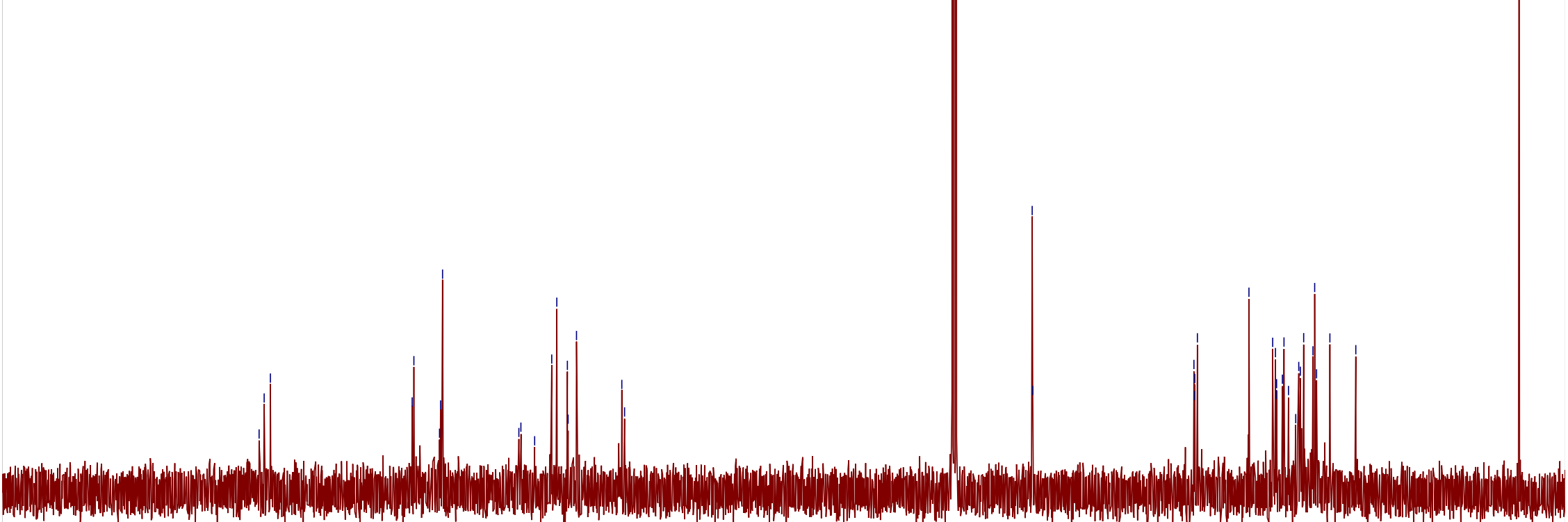

200190

180

170

160

150

140

130

120

$110 \quad 100$

f1 (ppm)

80

70

60

50

30

20

10

0 
AL-06-172.1.fid

1H AL-06-172
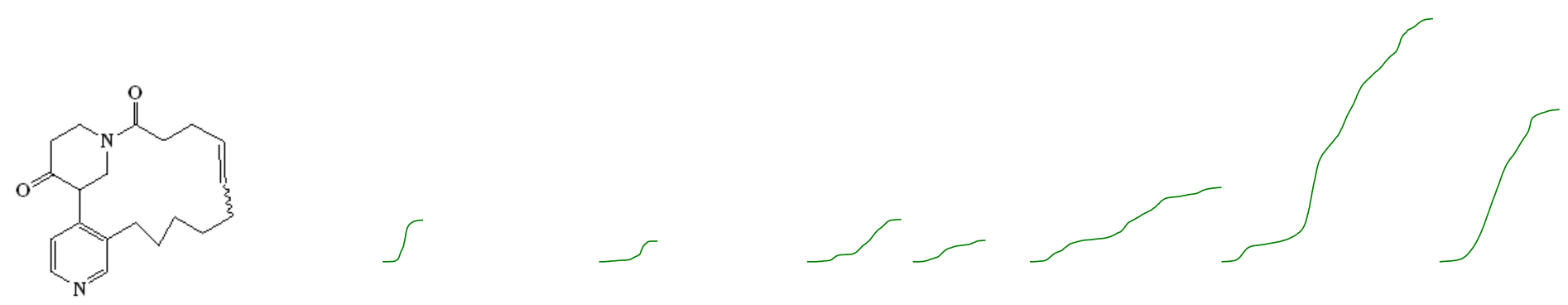

50

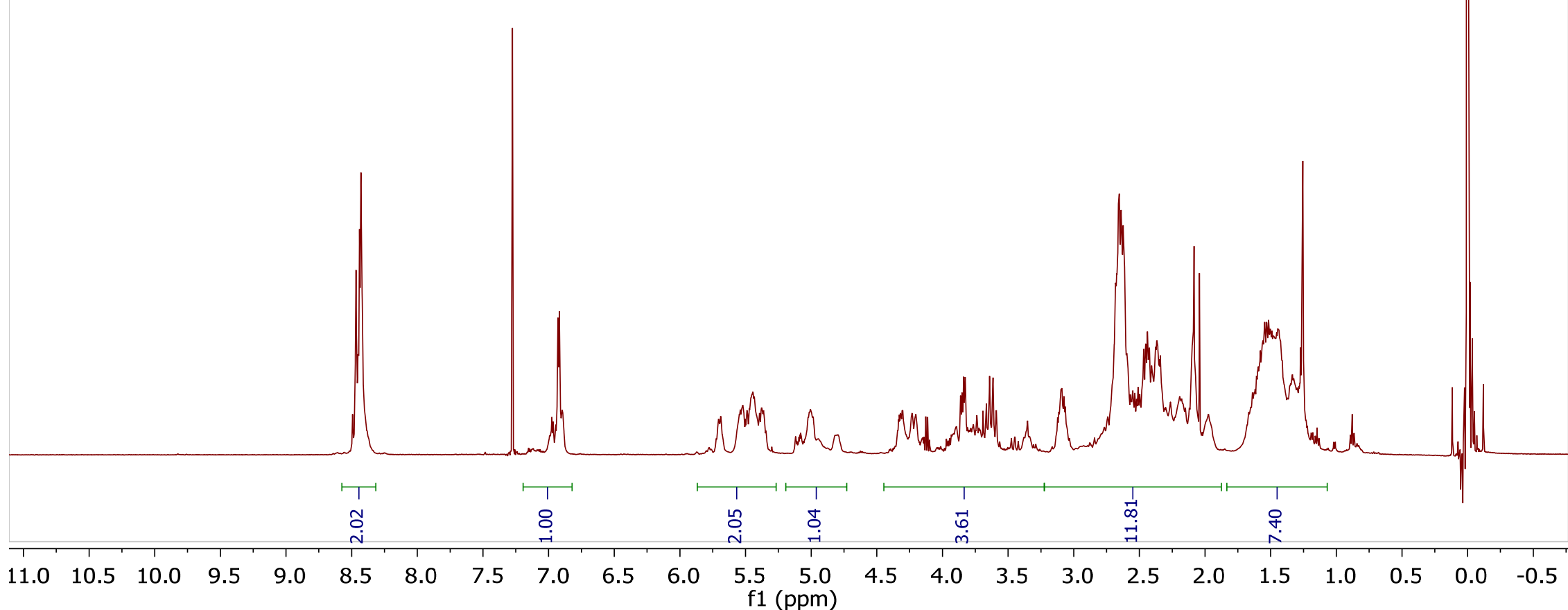


AL-06-172.2.fid

13C AL-06-172

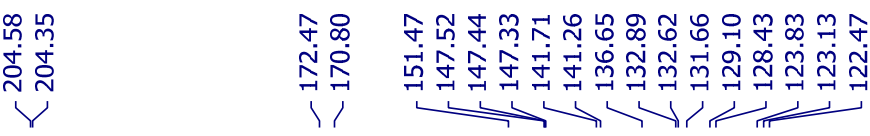

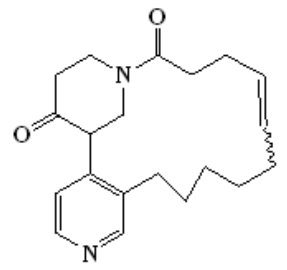

50

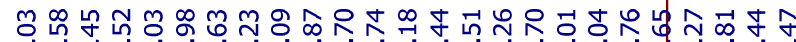
ก่

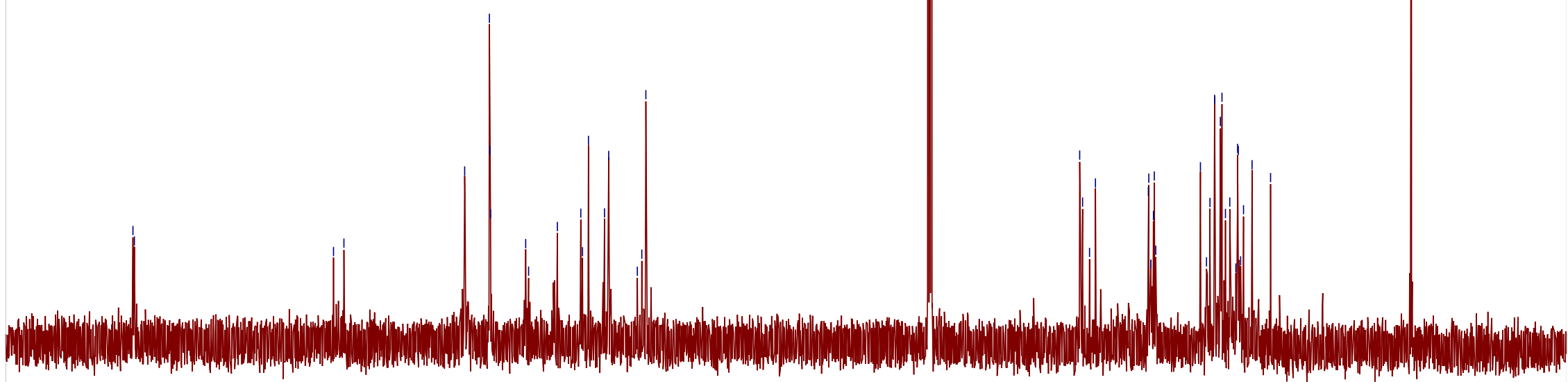

170160

$140 \quad 130$

120

$110 \underset{f 1}{100} 90$

80

$60 \quad 50$

40

$30 \quad 20$

10

$-20$ 
AL-11-82.1.fid

AL-11-82
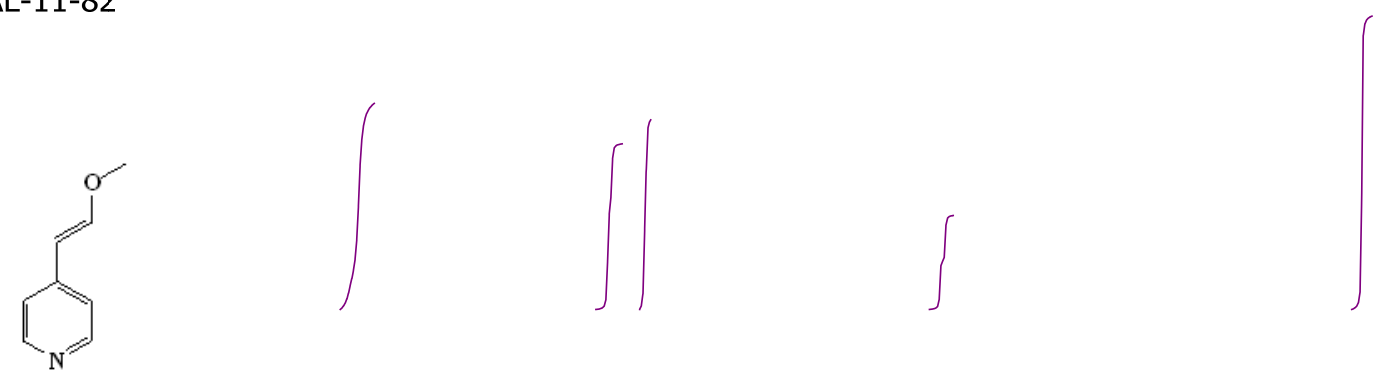

52

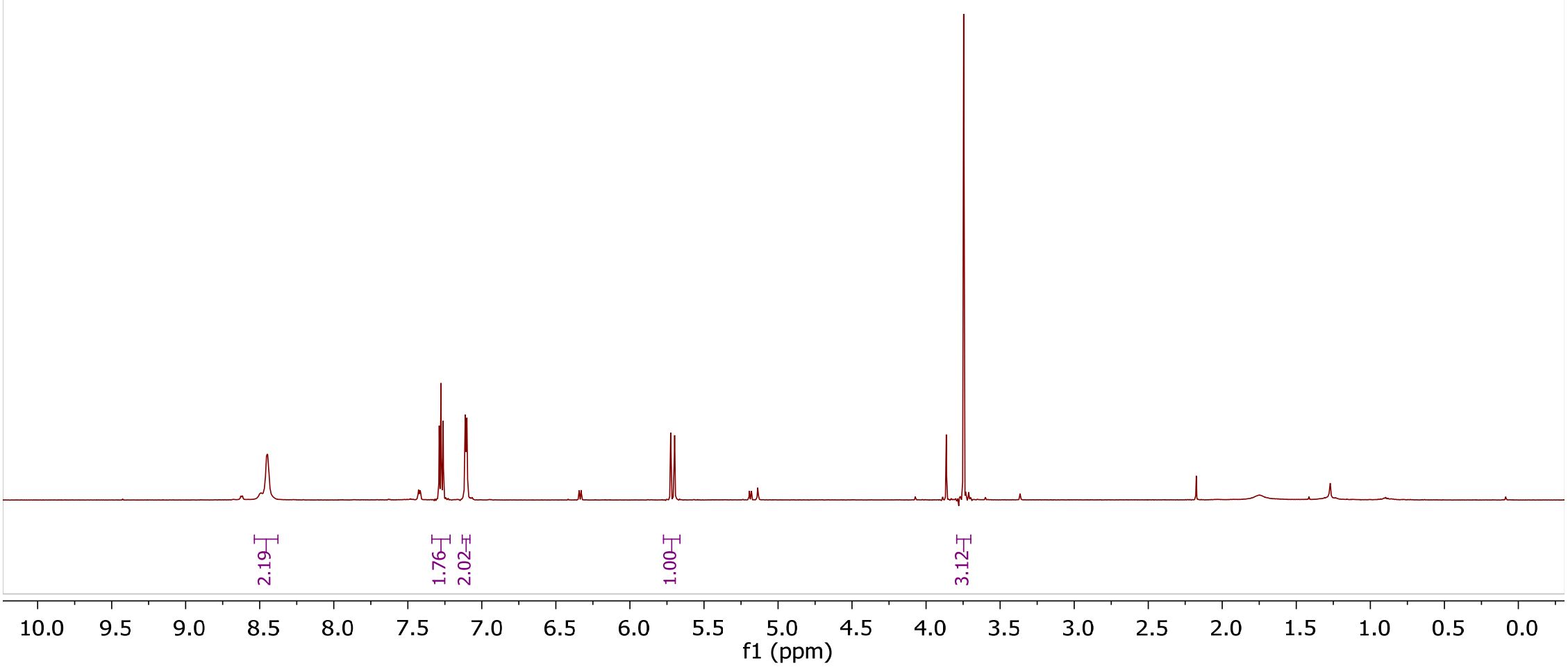




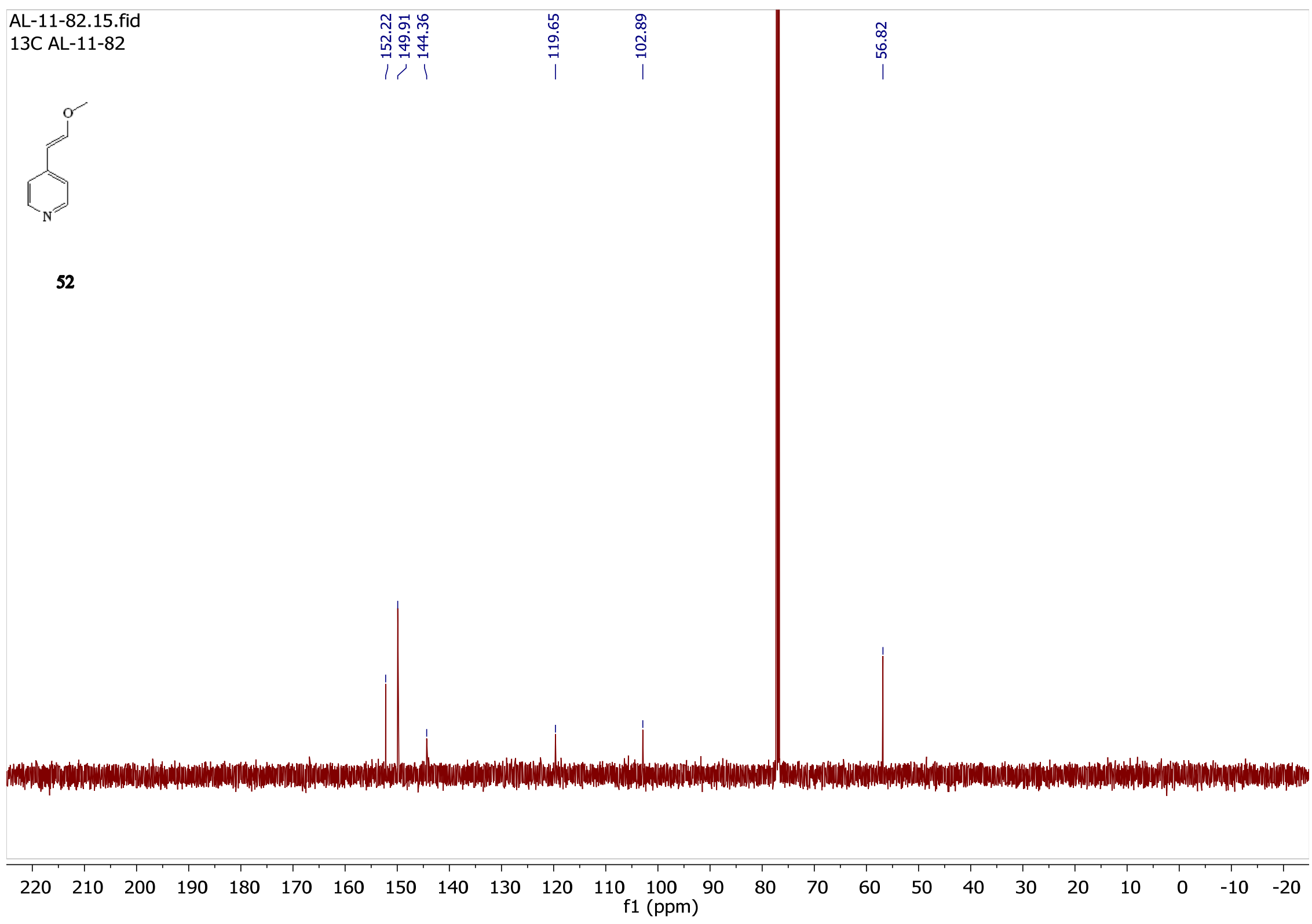


AL-10-206.1.fid

1H AL-10-206
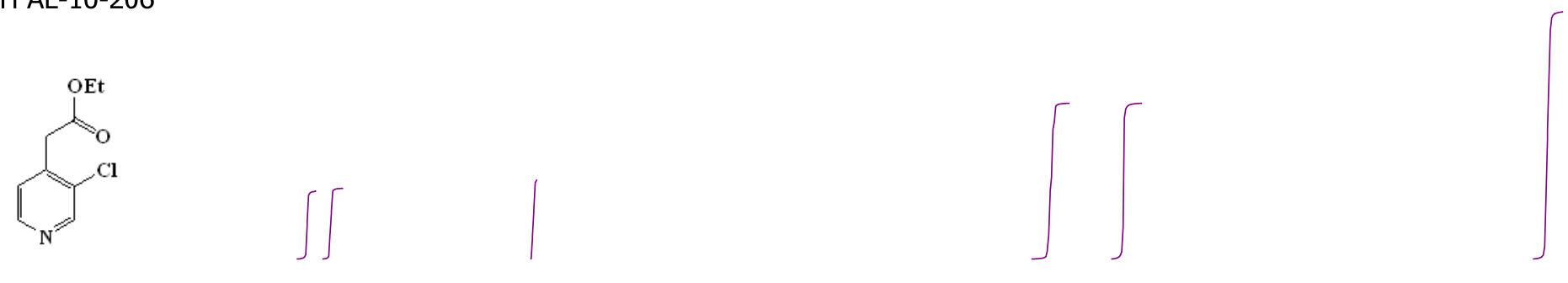

54
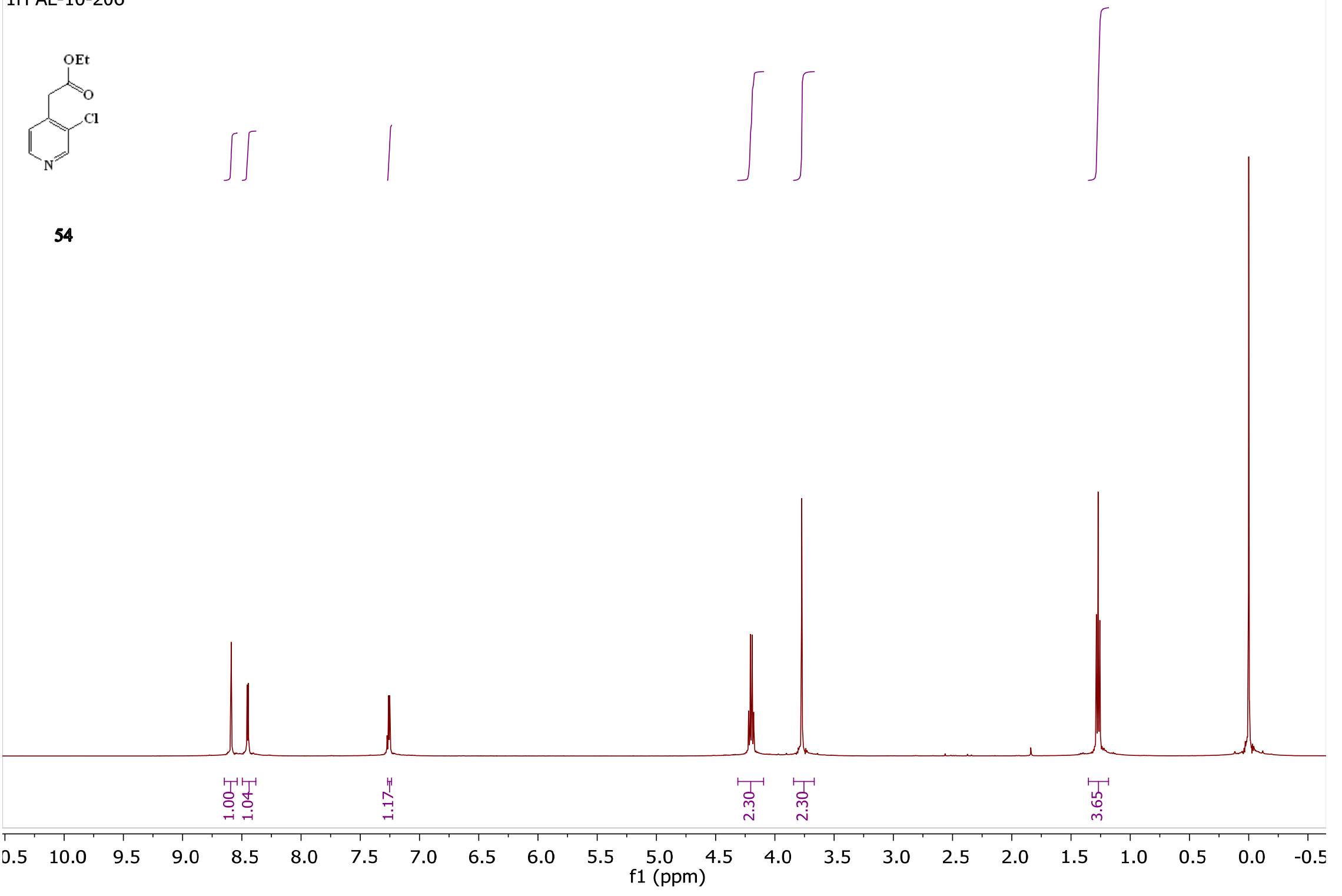
AL-10-206.2.fid

13C AL-10-206

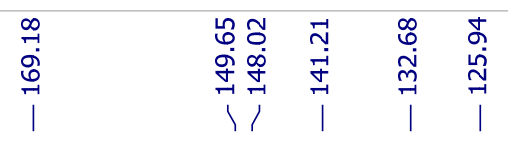

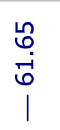

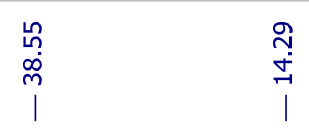

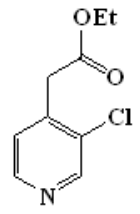

54

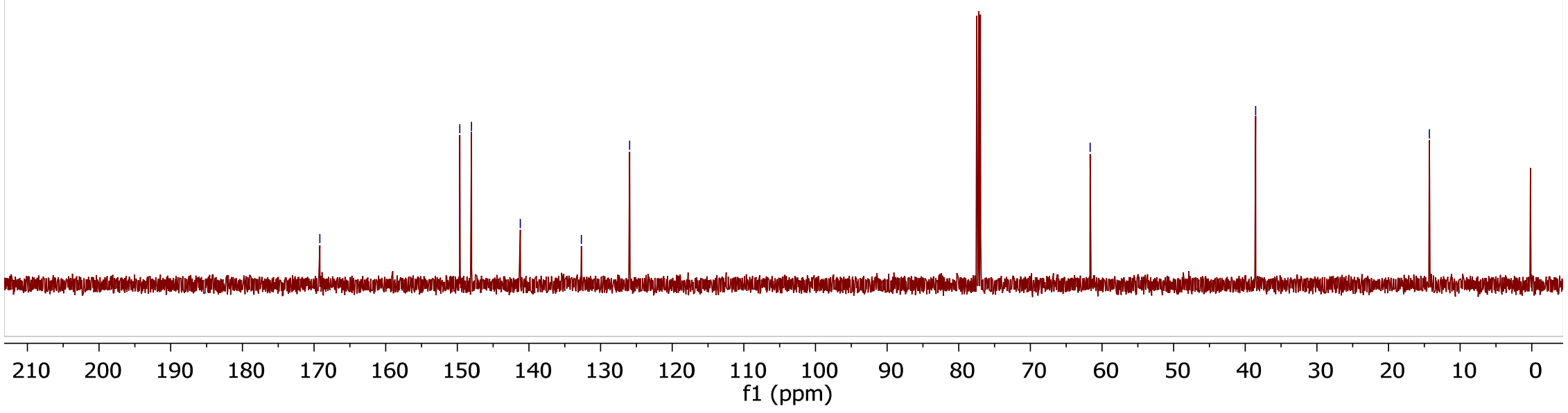


AL-10-294.1.fid

1H AL-10-294

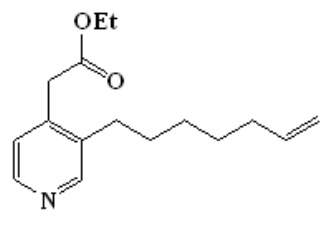

55
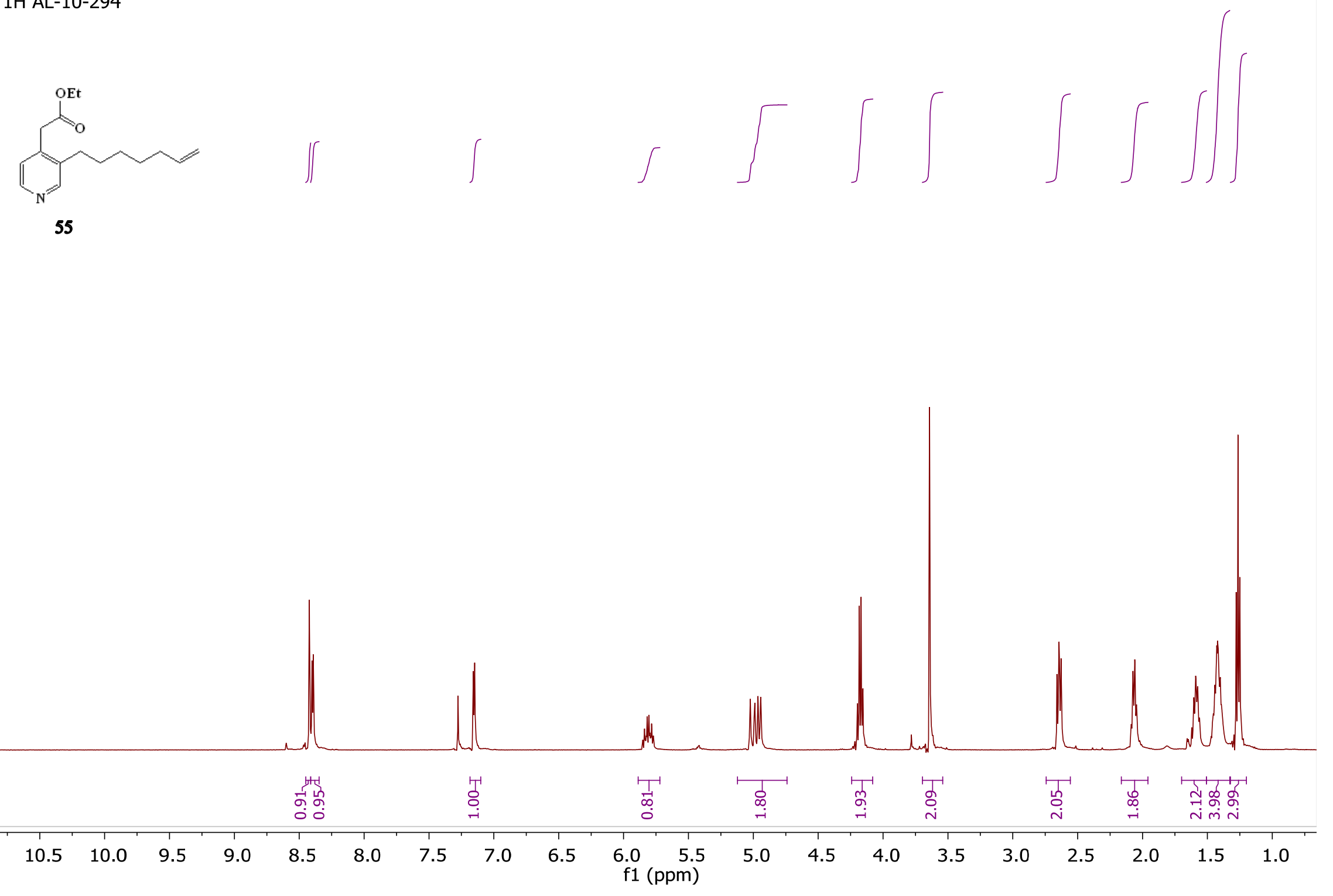


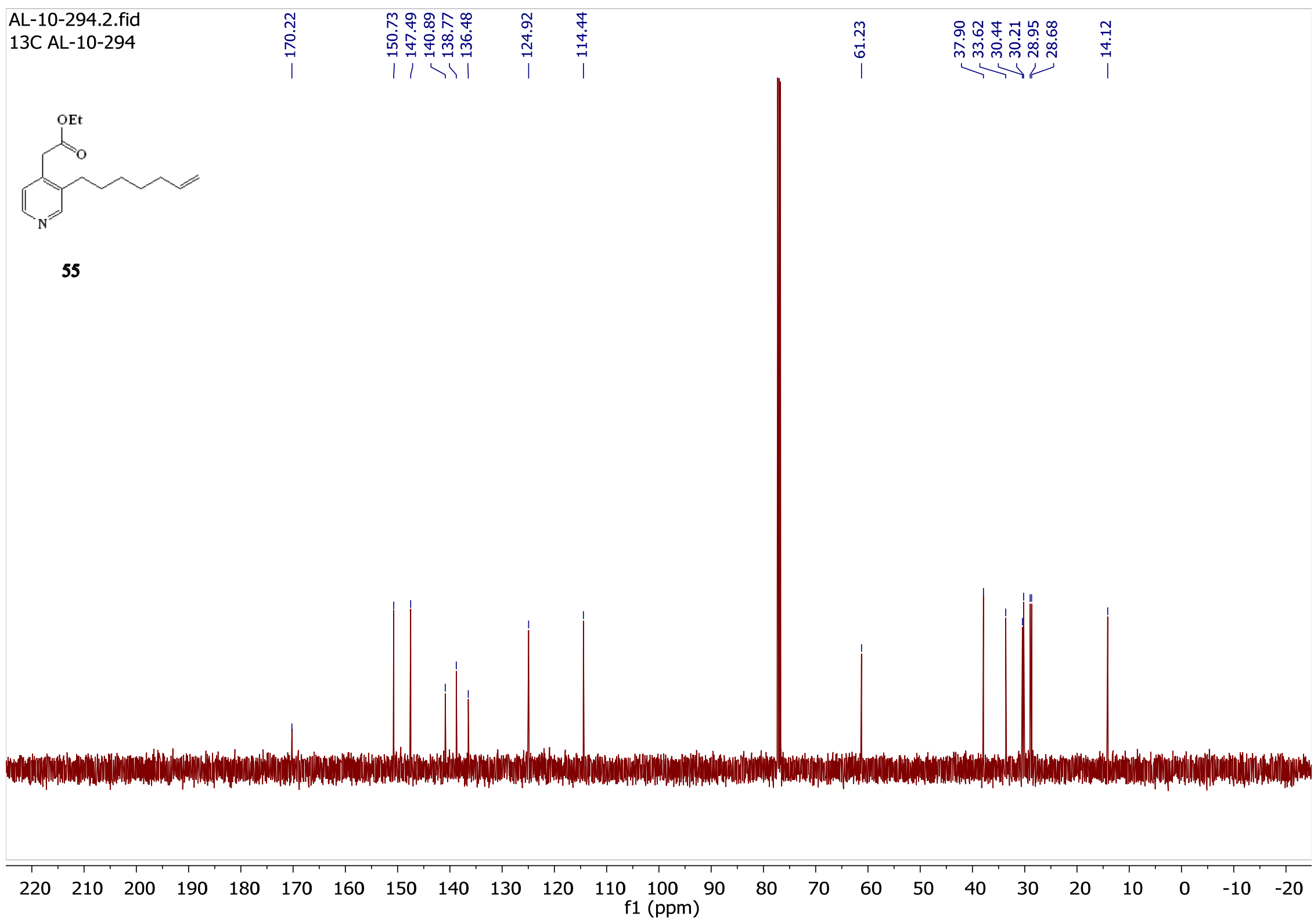


AL-10-210.1.fid

1H AL-10-210
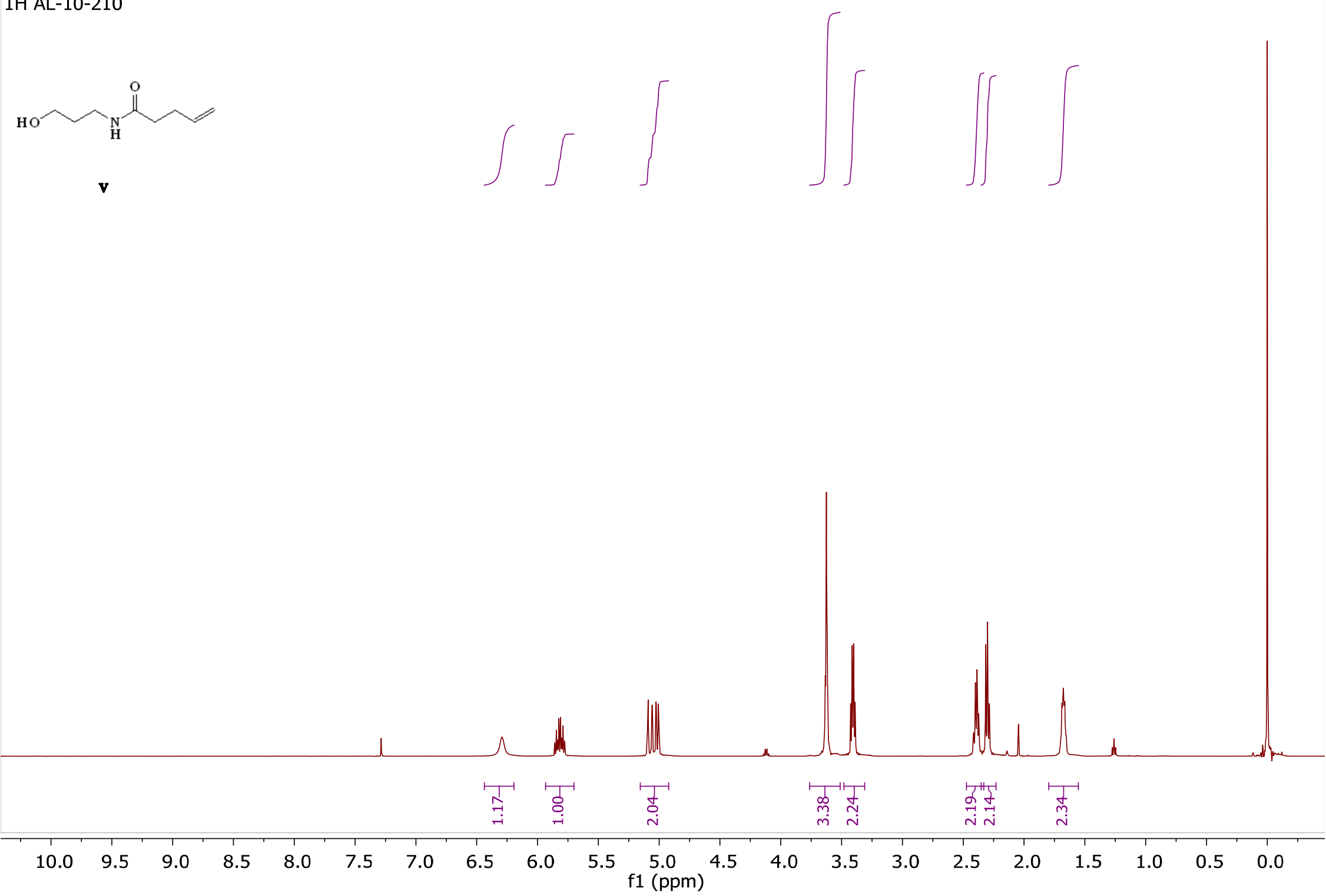


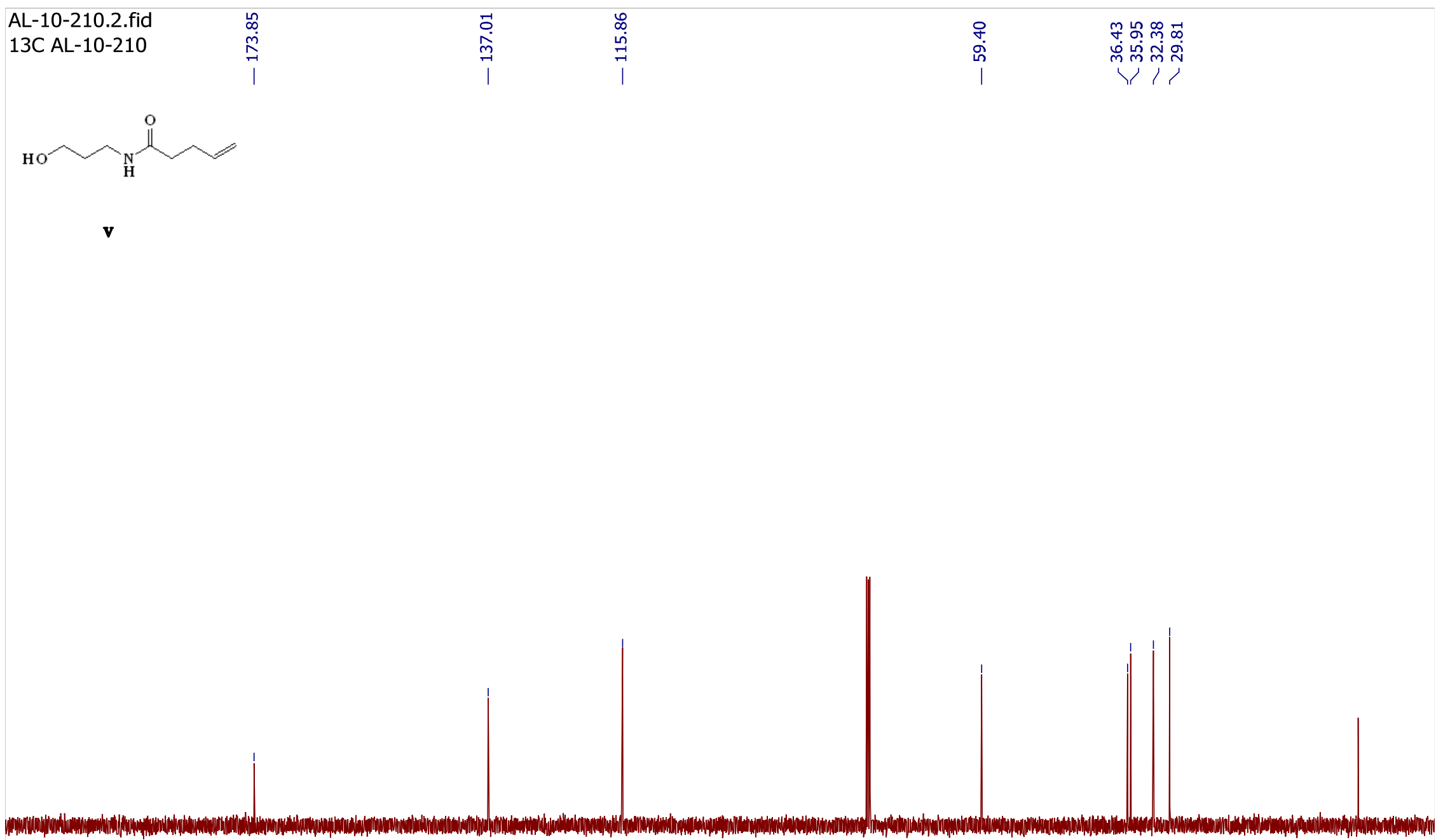

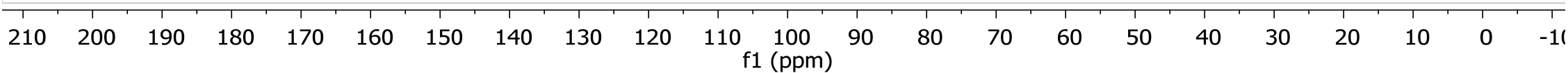


AL-10-172.1.fid

1H AL-10-172

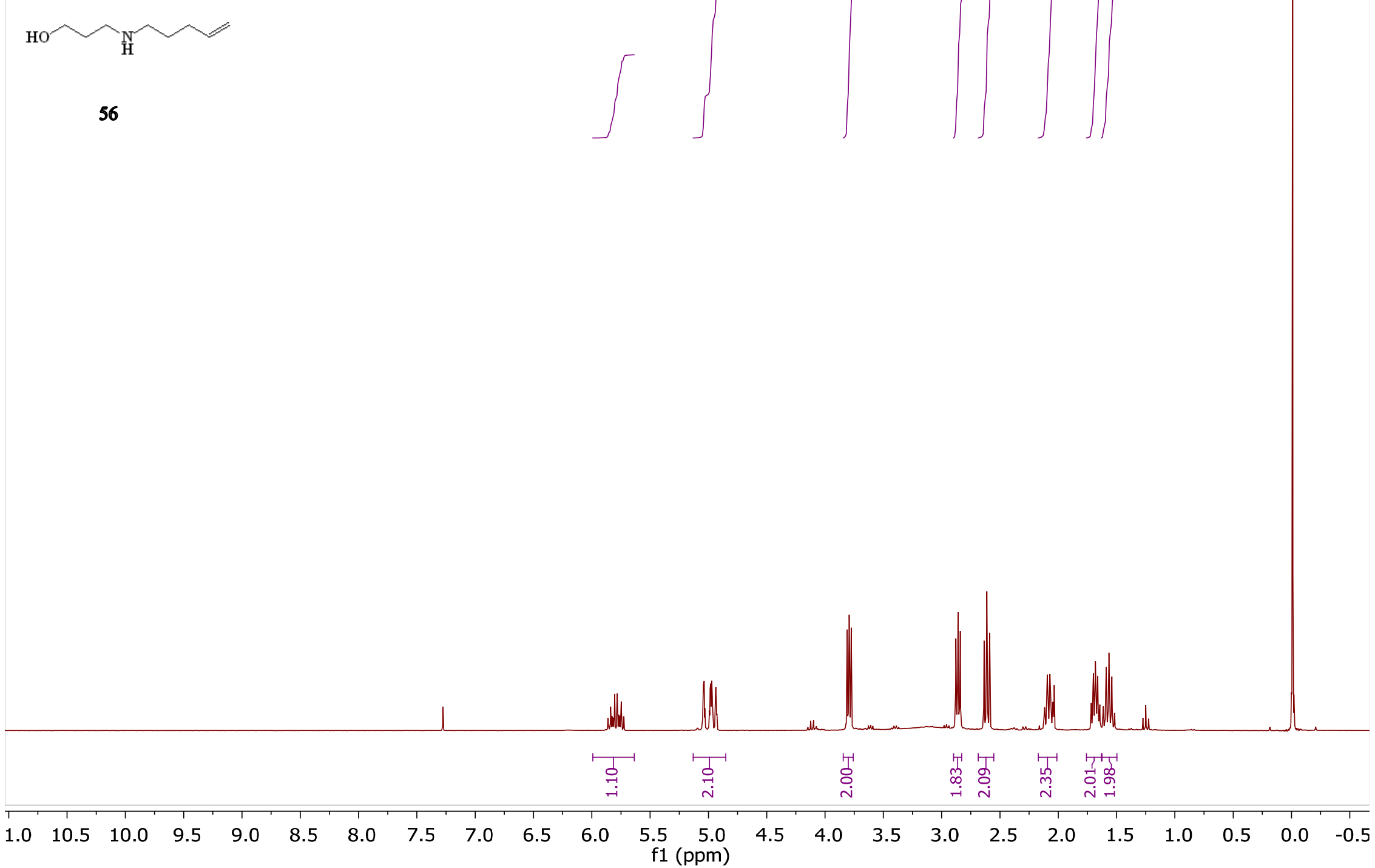




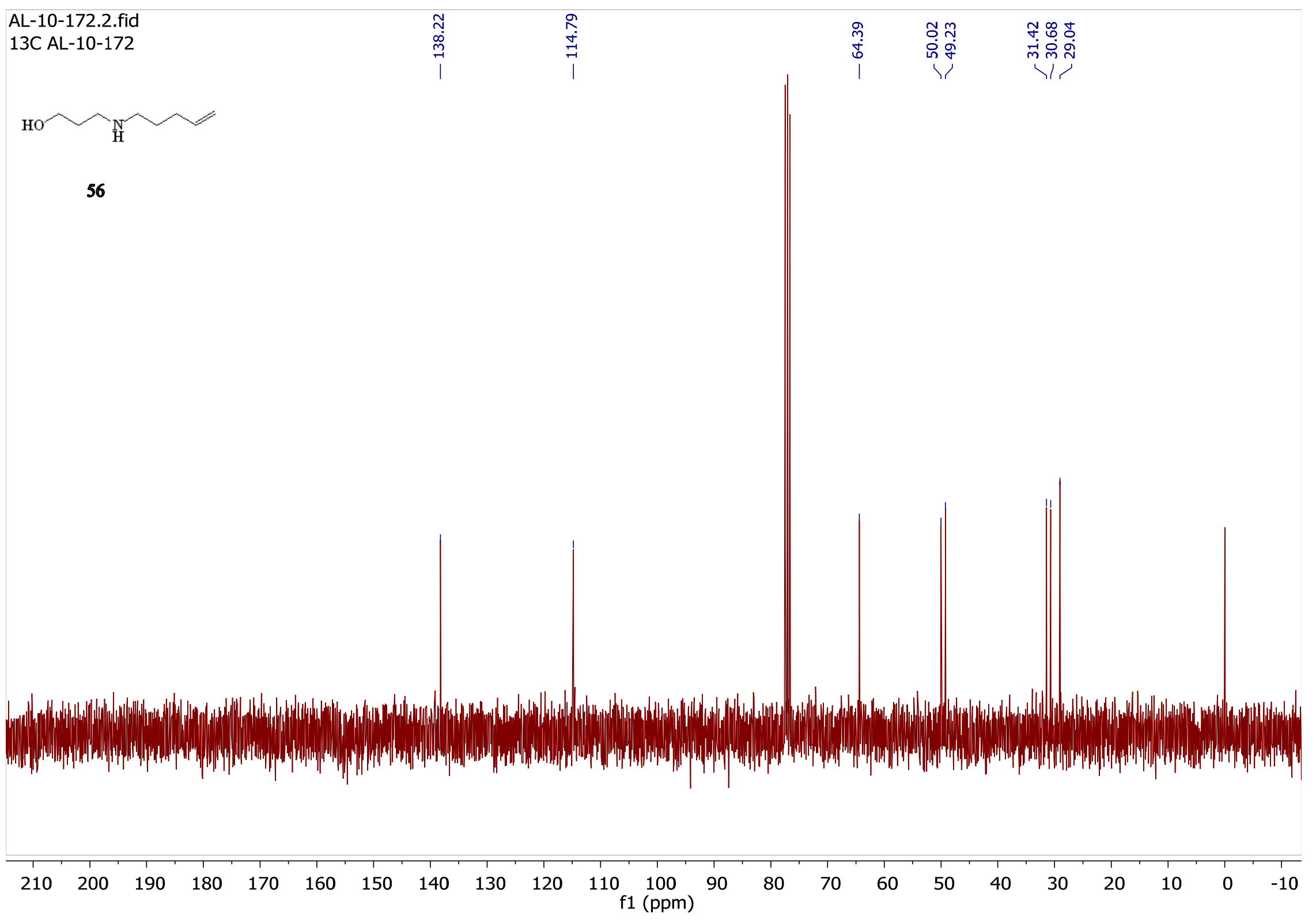


AL-10-254.1.fid

1H AL-10-254
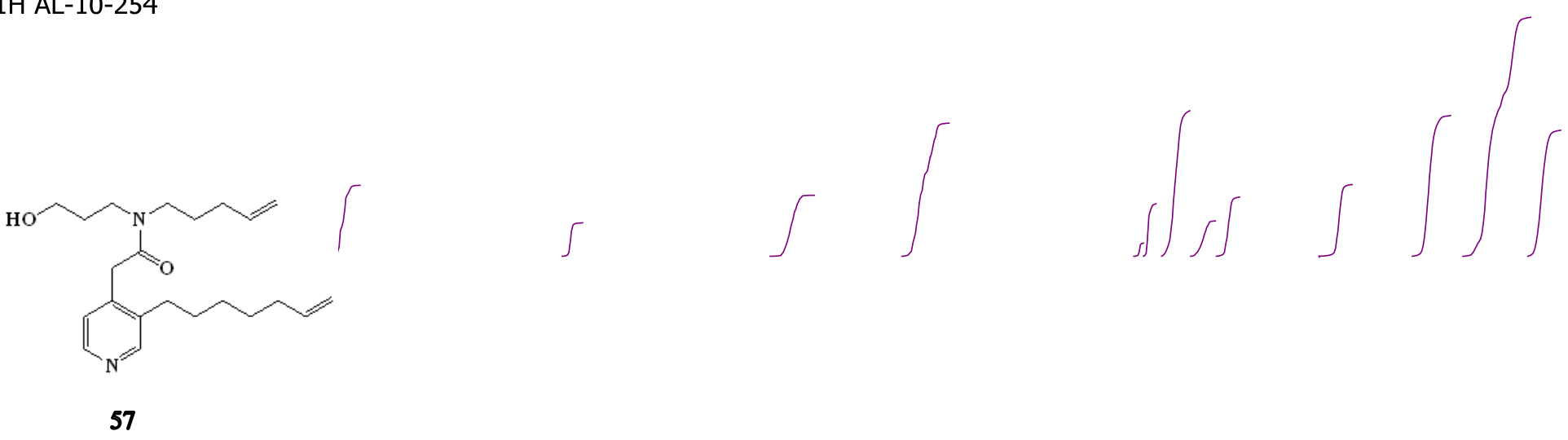

57

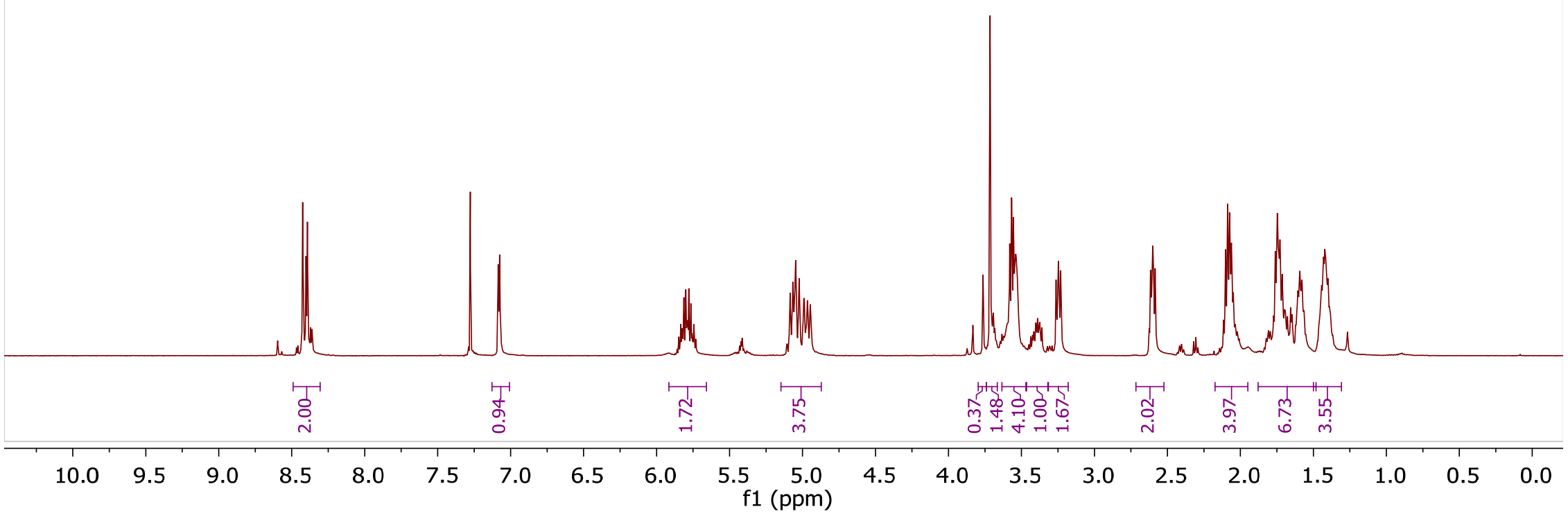




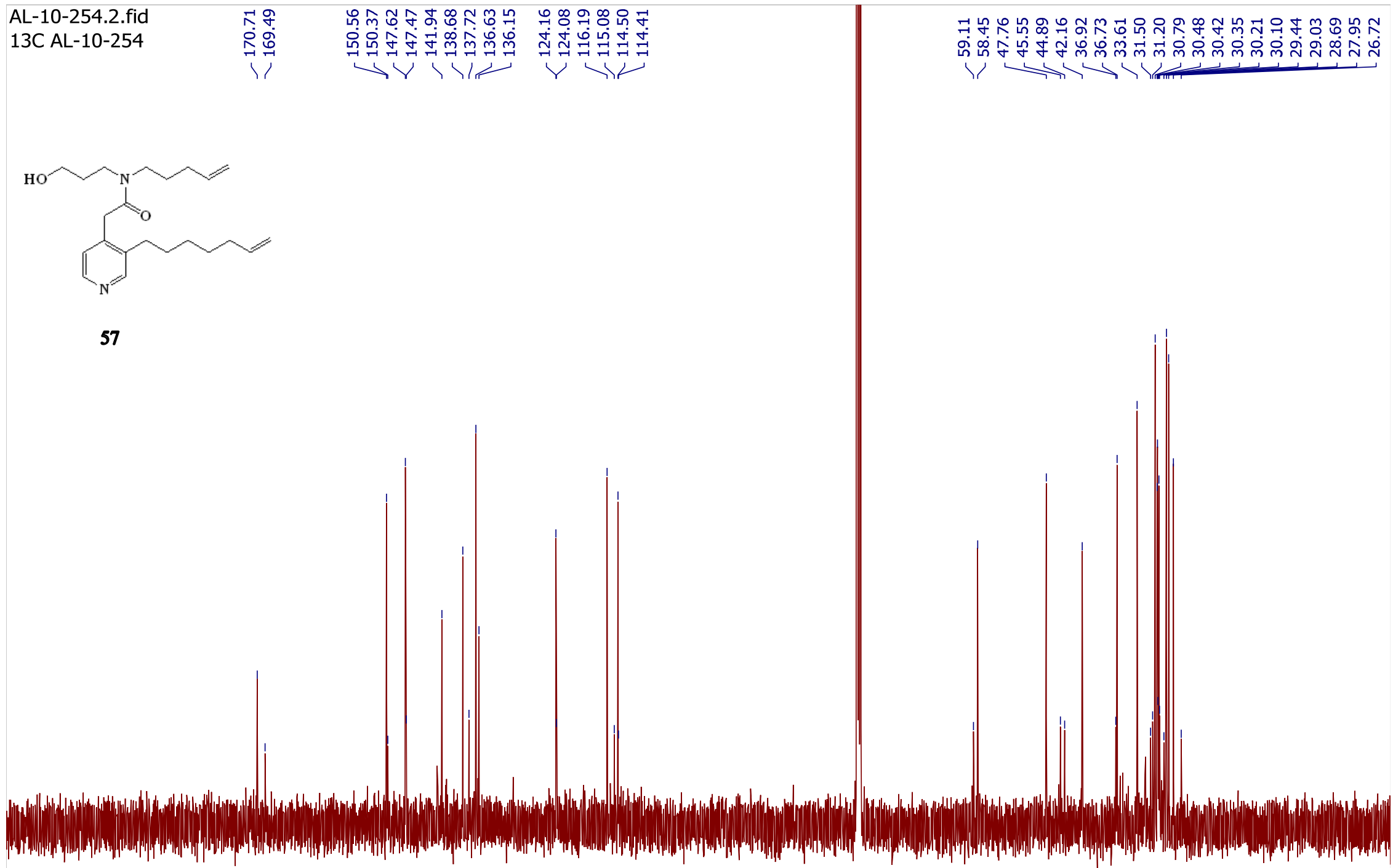

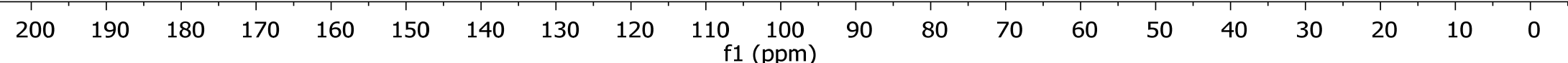


AL-10-292.1.fid

1H AL-10-292

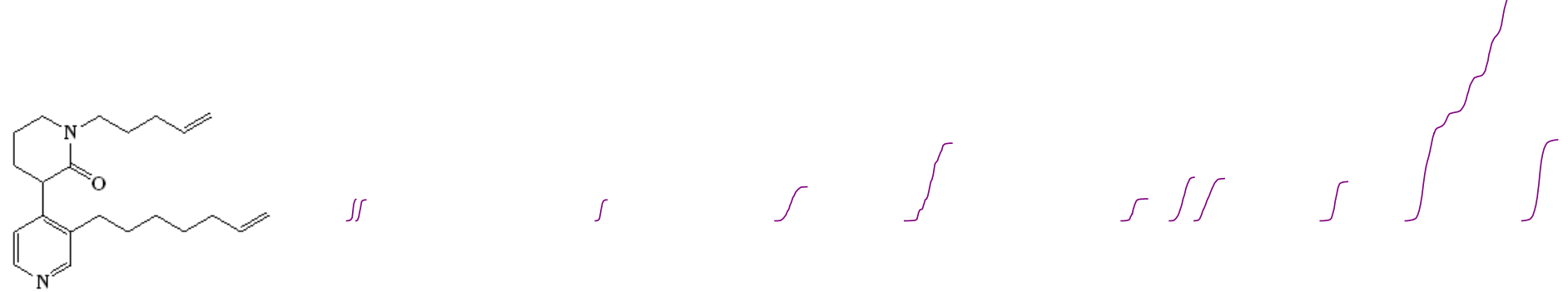

58

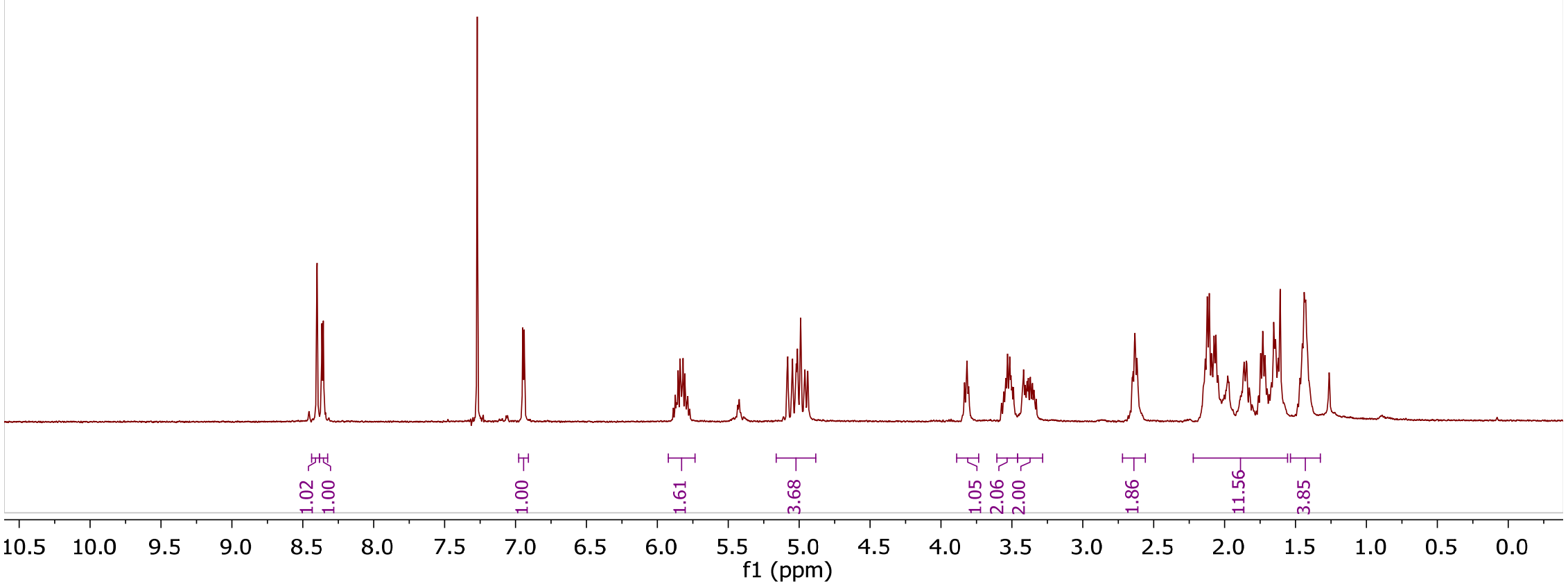




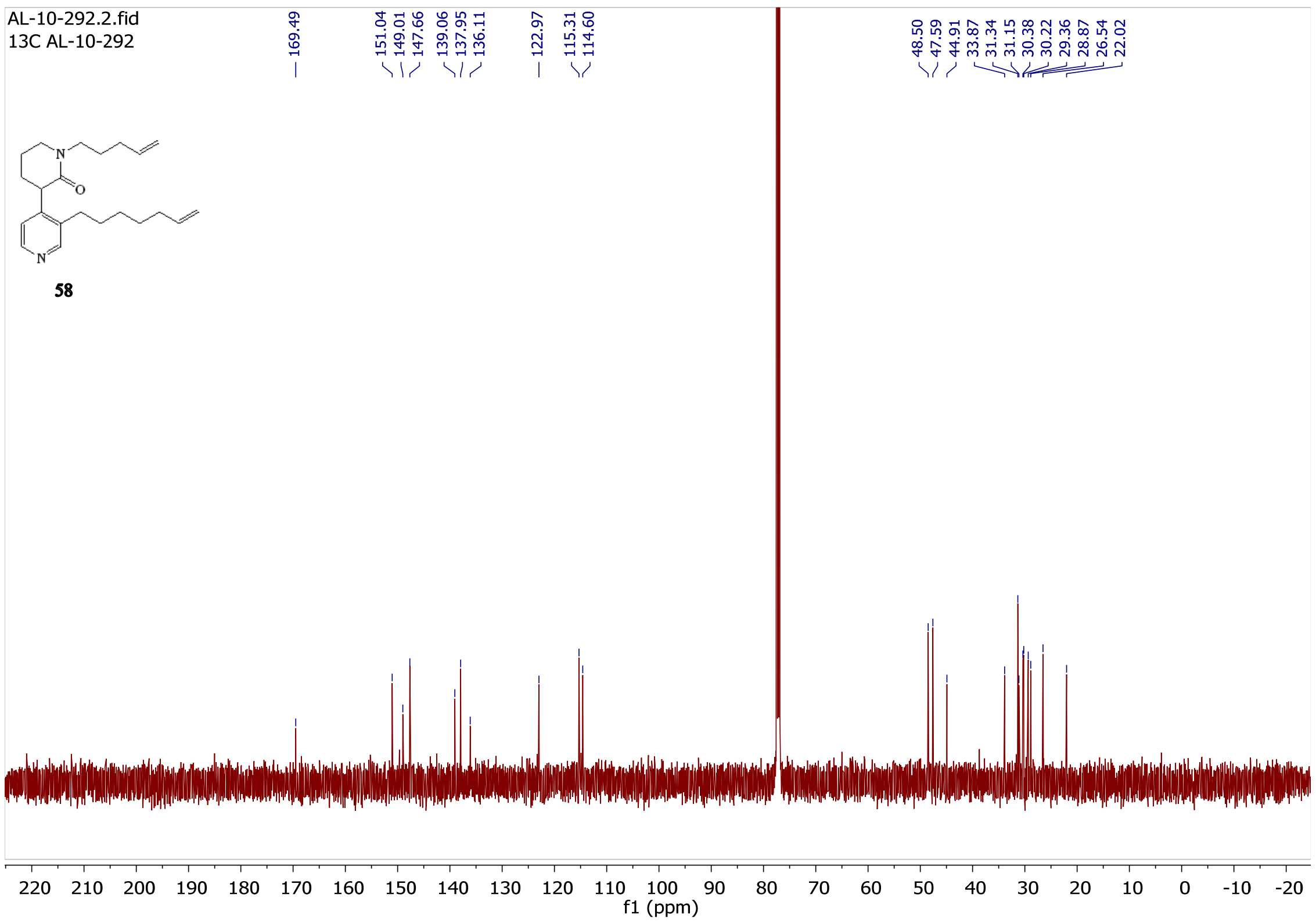


AL-10-298 After RCM.1.fid

1H AL-10-298 After RCM

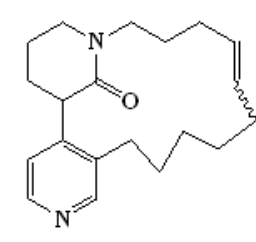

vi
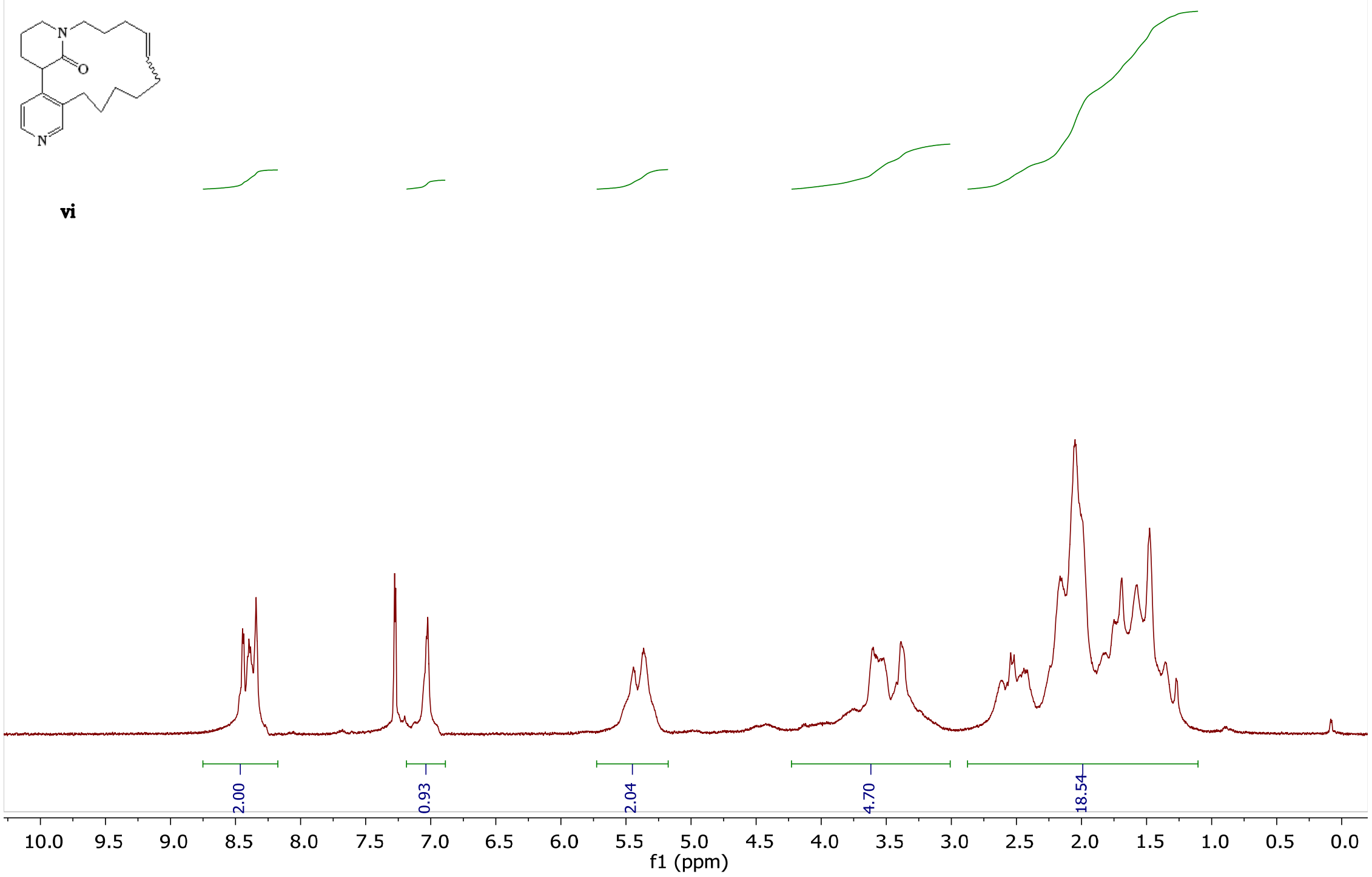
AL-10-298 After RCM.2.fid 13C AL-10-298 After RCM

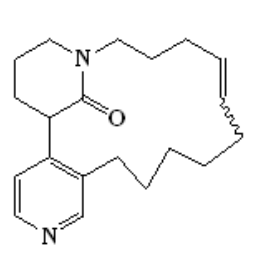

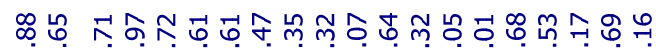

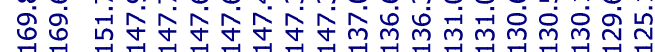

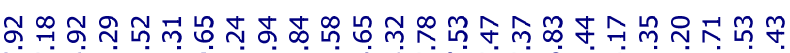

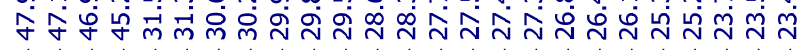

vi
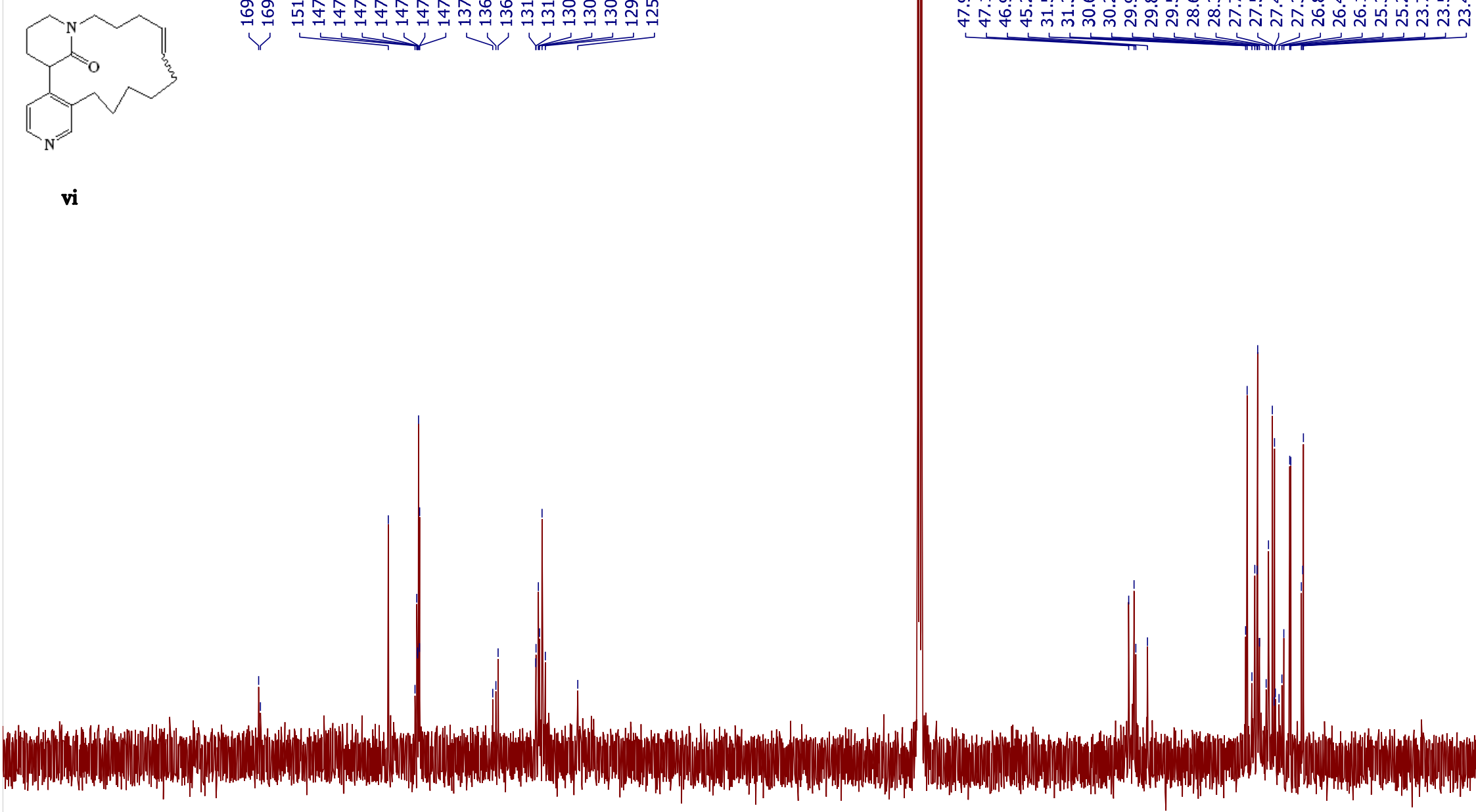
AL-10-298 retake.1.fid

1H AL-10-298 retake
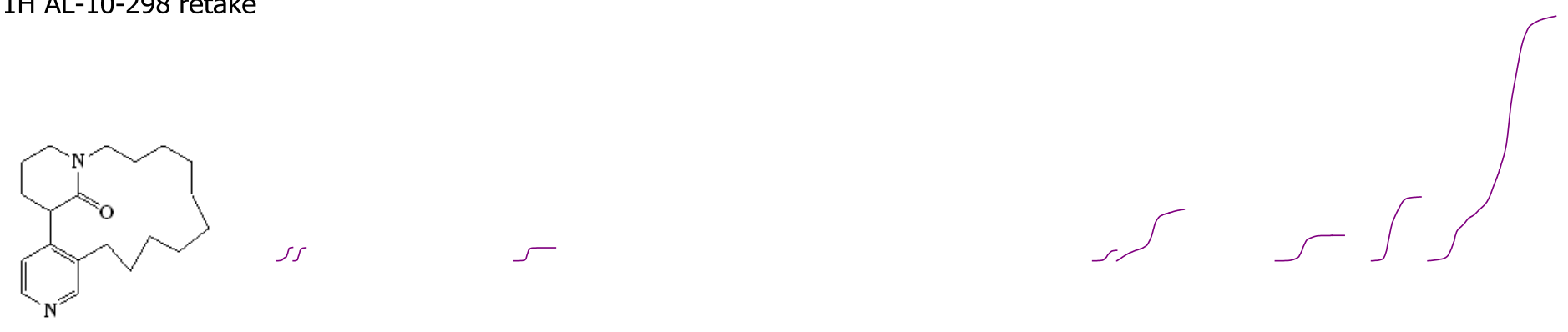

59

$$
\begin{array}{|c|}
\hline C(d) \\
8.37 \\
\hline B(m) \\
8.45 \\
\hline
\end{array}
$$

7.05

$$
\begin{array}{|l|}
\hline \begin{array}{c}
H(\mathrm{~m}) \\
3.46
\end{array} \\
\hline D(t) \\
3.70 \\
\hline
\end{array}
$$

\begin{tabular}{|c|c|c|}
\hline$E(m)$ & $F(m)$ & $G(m)$ \\
2.56 & 2.05 & 1.45 \\
\hline
\end{tabular}
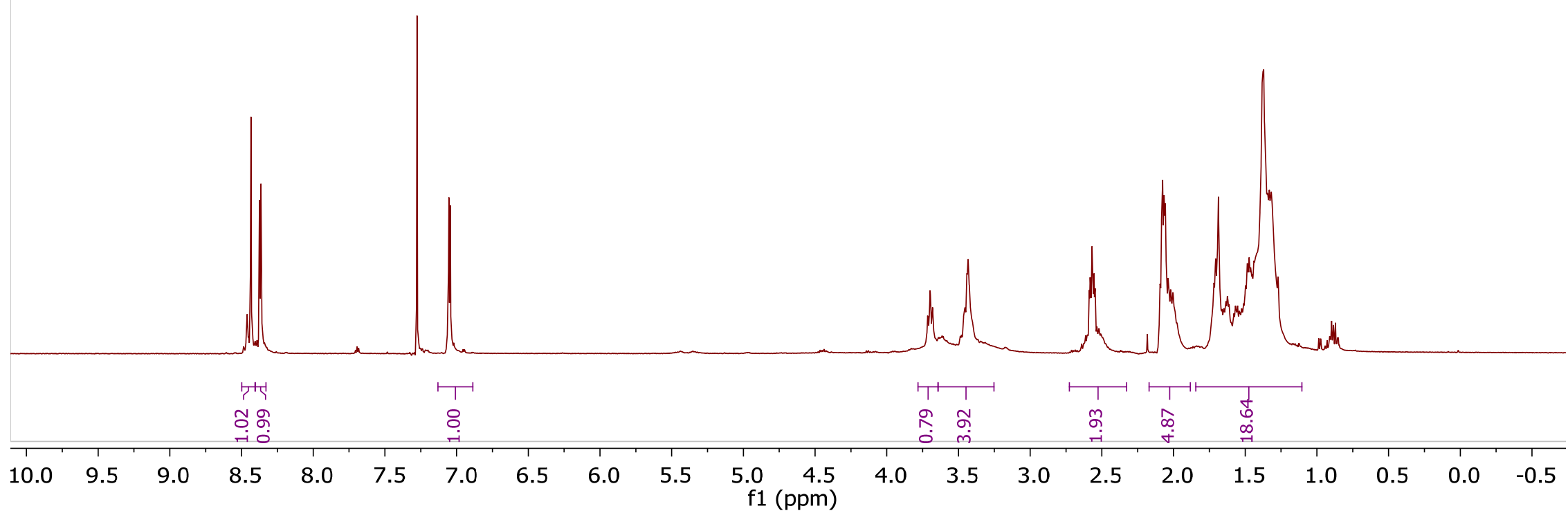
AL-10-298 retake.2.fid 13C AL-10-298 retake

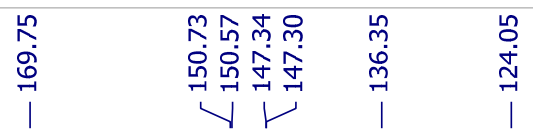

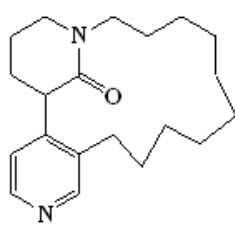

59

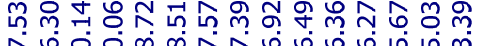

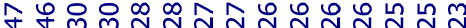

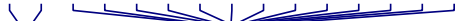

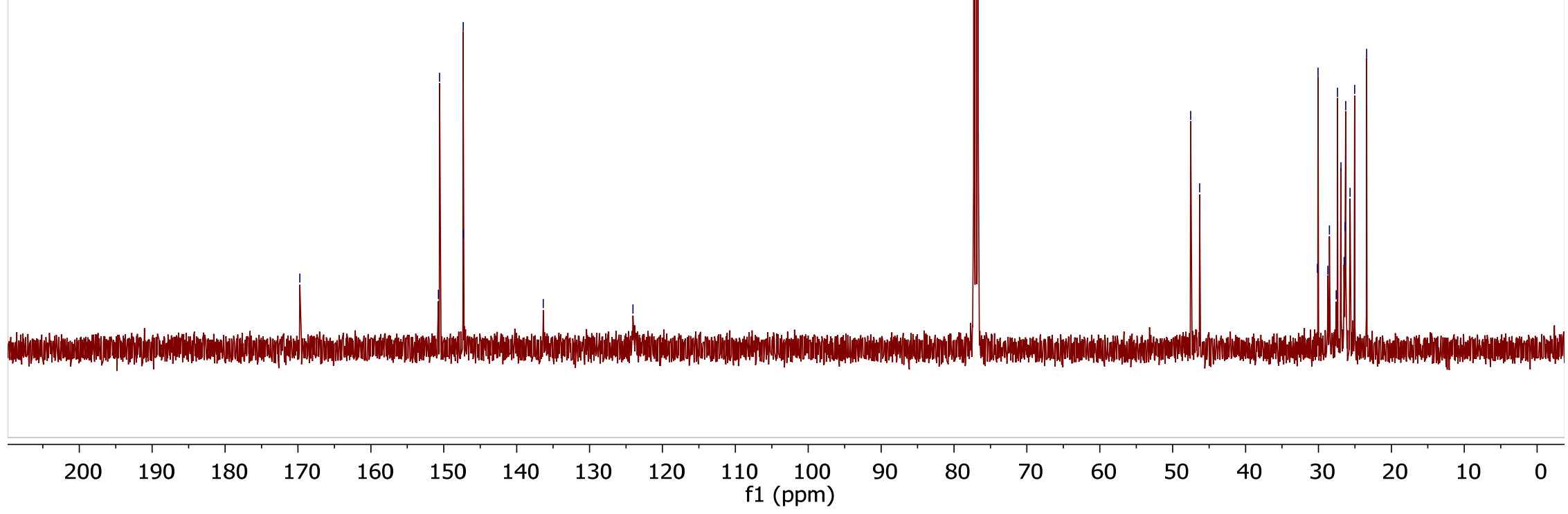


AL-10-242 Anhydrobase.1.fid

1H AL-10-242 Anhydrobase
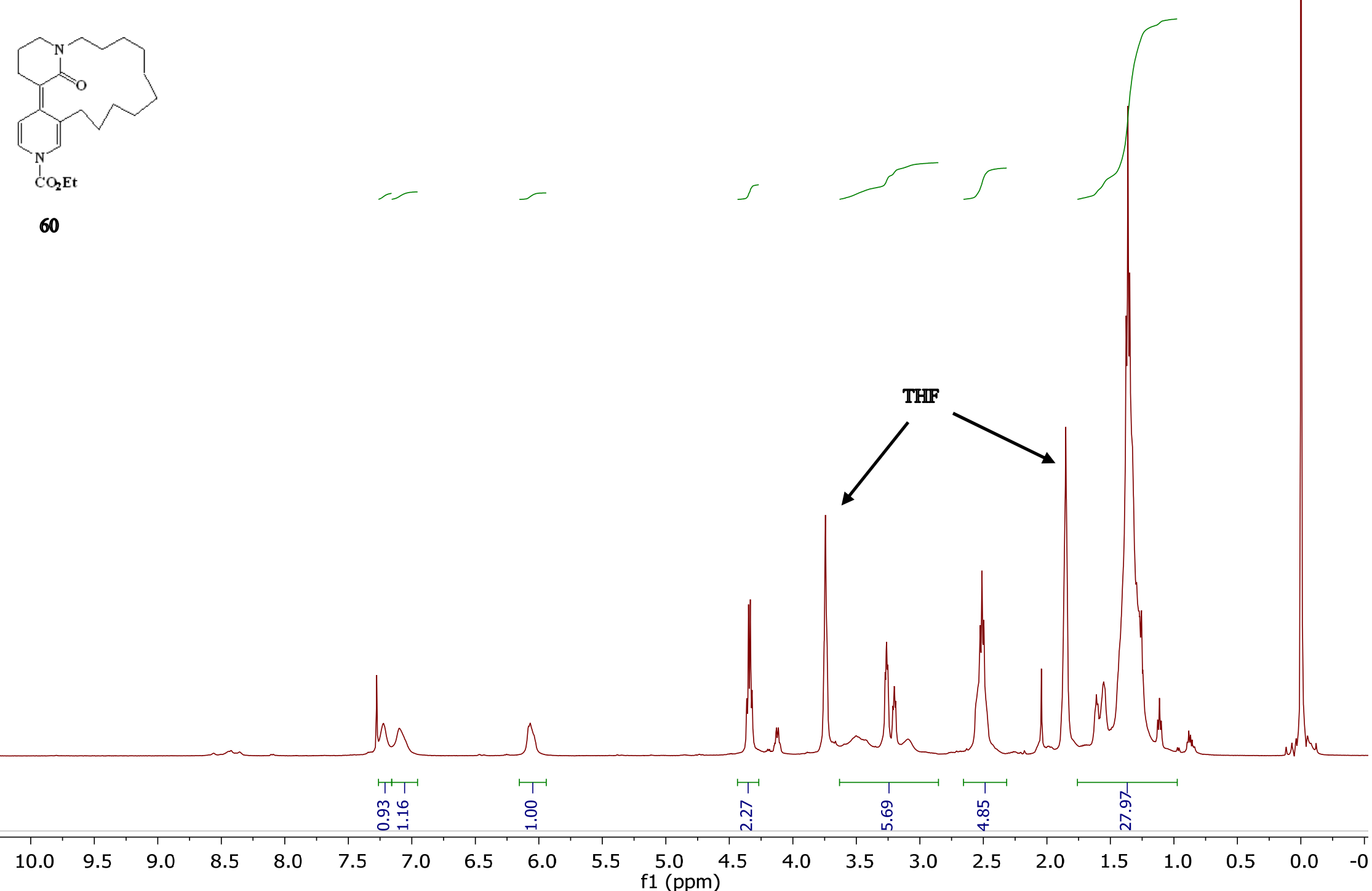
AL-11-14/61
1H AL-11-14

Temp - $298 \mathrm{~K}$

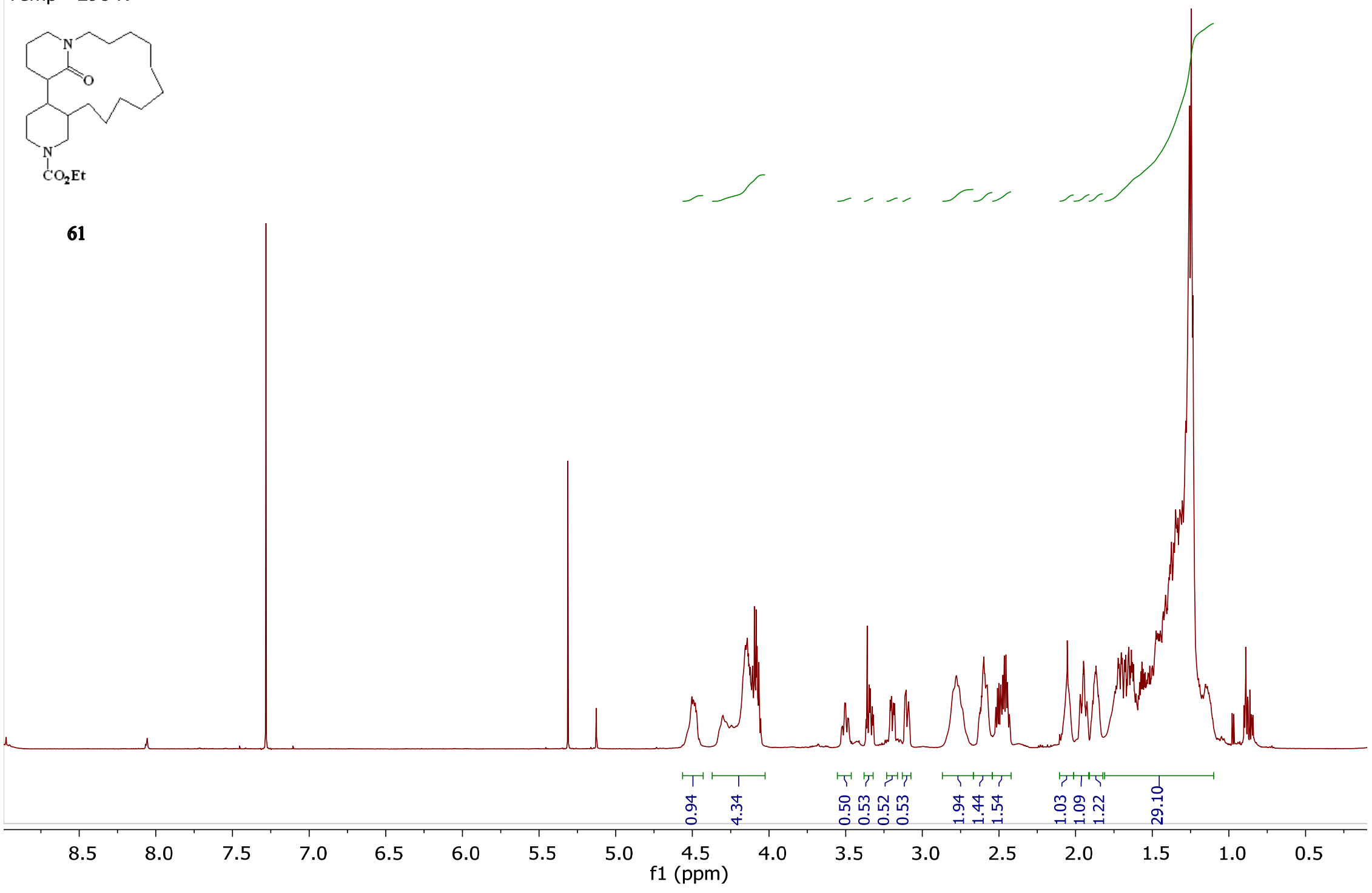


AL-11-14/6

13C AL-11-14

temp $=298 \mathrm{k}$

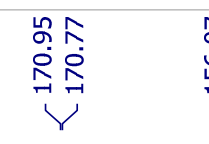

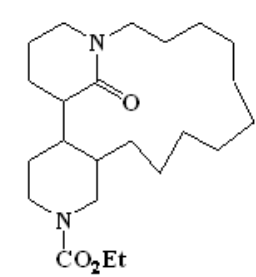

61

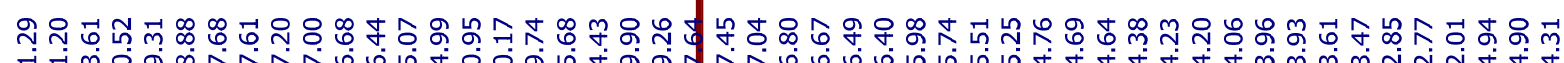

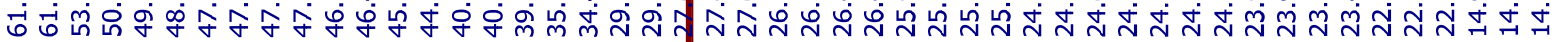

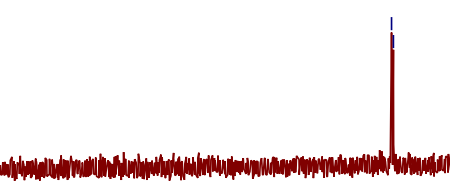

tran 似

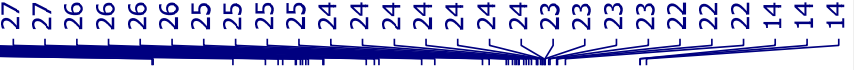

\begin{tabular}{|c|c|c|c|c|c|c|c|c|c|c|c|c|c|c|c|c|c|c|c|c|}
\hline 1 & 1 & 1 & 1 & 1 & 1 & $T$ & 1 & 1 & 1 & $T$ & 1 & $T$ & $T$ & T & T & $T$ & $T$ & $T$ & $T$ & 1 \\
\hline 200 & 190 & 180 & 170 & 160 & 150 & 140 & 130 & 120 & 110 & $\begin{array}{c}100 \\
(\mathrm{ppm})\end{array}$ & 90 & 80 & 70 & 60 & 50 & 40 & 30 & 20 & 10 & 0 \\
\hline
\end{tabular}


AL-11-14/67

13C DEPT AL-11-14

temp $=298 \mathrm{k}$

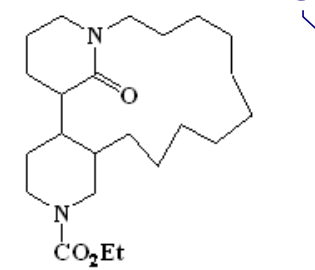

ํำ

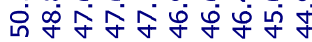

ํ. 숭

$\stackrel{\infty}{:}$

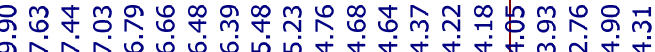

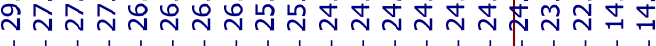

1 1

61

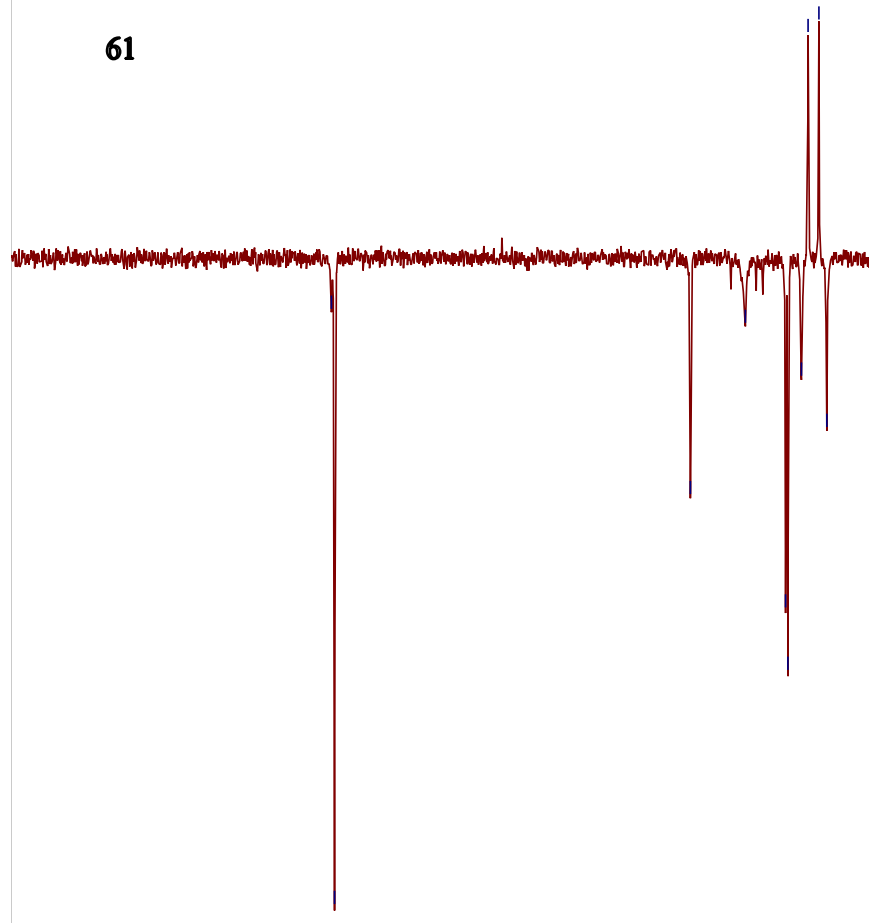

$70 \quad 68$

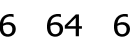

